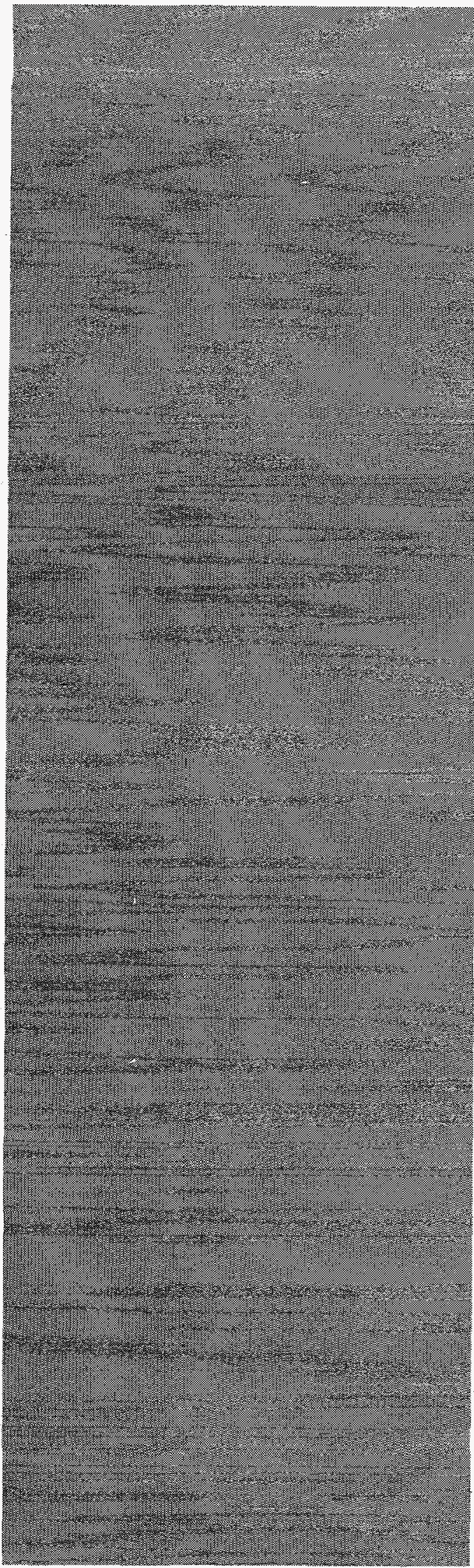

\title{
Use of Ultracold Neutrons for \\ Condensed-Matter Studies
}

\section{ECEIVED JUH 25 A97 OSTI}

\section{Los Alamos}

Los Alamos National Laboratory is operated by the University of California for the United States Department of Energy under contract W-7405-ENG-36. 


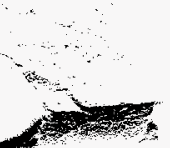

Edited by inana Buican, Group CIC-1

An Affirmative Action/Equal Opportunity Employer

This report was prepared as an account of work sponsored by an agency of the United States Government. Neither The Regents of the University of California, the United States

Government nor any agency thereof, nor any of their employees, makes any warranty, express or implied, or assumes any legal liability or responsibility for the accuracy, completeness, or usefulness of any information, apparatus, product, or process disclosed, or represents that its use would not infringe privately owned rights. Reference herein to any specific commercial product, process, or service by trade name, trademark, manufacturer, or otherwise, does not necessarily constitute or imply its endorsement, recommendation, or favoring by The Regents of the University of California, the United States Government, or any agency thereof. The views and opinions of authors expressed herein do not necessarily state or reflect those of The Regents of the University of California, the United States Government, or any agency thereof. Los Alamos National Laboratory strongly supports academic freedom and a researcher's right to publish; as an institution, however, the Laboratory does not endorse the viewpoint of a publication or guarantee its technical correctness. 


\title{
Use of Ultracold Neutrons for Condensed-Matter Studies
}

\author{
André Michaudon
}

\section{DISCLAIMER}

This report was prepared as an account of work sponsored by an agency of the United States Government. Neither the United States Government nor any agency thereof, nor any of their employees, makes any warranty, express or implied, or assumes any legal liability or responsibility for the accuracy, completeness, or usefulness of any information, apparatus, product, or process disclosed, or represents that its use would not infringe privately owned rights. Reference herein to any specific commercial product, process, or service by trade name, trademark, manufacturer, or otherwise does not necessarily constitute or imply its endorsement, recommendation, or favoring by the United States Government or any agency thereof. The views and opinions of authors expressed herein do not necessarily state or reflect those of the United States Government or any agency thereof.

\section{MASTER}





\section{DISCLAMIER}

Portions of this document may be illegible in electronic image products. Images are produced from the best available original document. 


\section{CONTENTS}

I. INTRODUCTION

II. BASIC PROPERTIES OF ULTRACOLD NEUTRONS …………………….... 2

III. SOME FUNDAMENTALS OF NEUTRON REFLECTIVITY ………............ 4

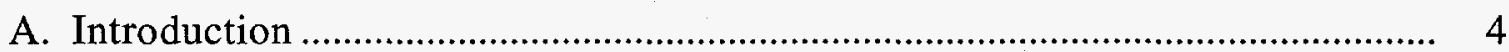

B. Neutron Reflectivity from Nonmagnetic Materials .............................................. 6

1. Reflectivity from an ideal surface ……......................................................... 6

2. Reflectivity from a plane surface with a soft potential .................................. 6

3. Reflectivity from rough surfaces .................................................................. 7

4. Reflectivity from gratings …………………………................................. 8

5. Reflectivity from single-layer films .............................................................. 9

6. Reflectivity from multilayer surfaces ............................................................. 9

7. Quasi-bound macroscopic states of neutrons in matter.................................. 10

C. Neutron Reflectivity from Magnetic Materials ................................................... 12

IV. QUASI-ELASTIC NEUTRON SCATTERING …....................................... 14

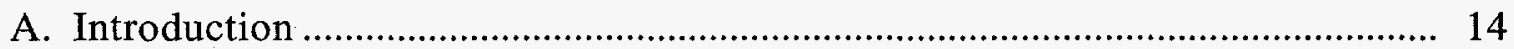

B. Inelastic-Scattering Formalism................................................................... 16

C. Dynamical Processes Studied in Quasi-Elastic Scattering .............................. 18

1. Introduction ......................................................................................... 18

2. Translational motions........................................................................ 19

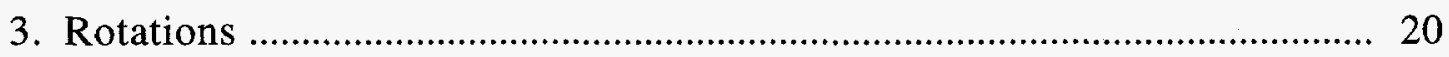

V. ULTRACOLD-NEUTRON GRAVITY INSTRUMENTS ………………........ 21

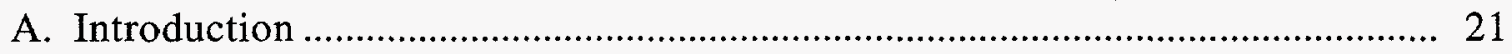

B. The UCN Gravity Diffractometer ................................................................... 21

C. The UCN Gravity Spectrometer ...................................................................... 23

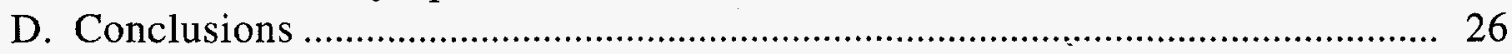

VI. SOME EXPERIMENTAL RESULTS IN NEUTRON REFLECTOMETRY ......................................................... 27

A. Reflection from a Smooth Planar Surface …………………………………….. 27

B. Effect of Roughness on the Reflectivity from and

Transmission through Planar Surfaces ........................................................... 27

C. Reflectivity from Gratings .......................................................................... 31

D. Reflectivity from Thin Films ............................................................................ 33

E. Reflectivity from and Transmission through Multilayer Systems ..................... 34

F. Reflectivity from Magnetic Samples ................................................................. 37

G. Quasi-Elastic Scattering ................................................................................ 40 
VII. COMPARISON OF ULTRACOLD-NEUTRON INSTRUMENTS WITH OTHER HIGH-RESOLUTION REFLECTOMETERS

A. Grazing-Angle Neutron Reflectometers ........................................................ 43

B. NSE Spectrometers ...................................................................................... 44

C. Optical Spectroscopy ……................................................................................ 50

D. Summary of the Performance of High-Resolution Instruments .......................... 50

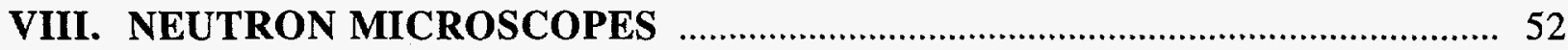

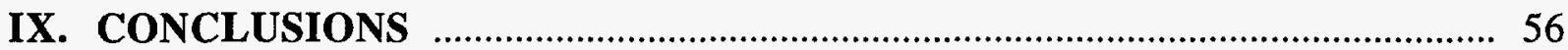

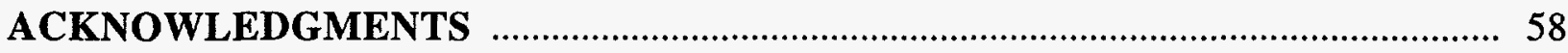

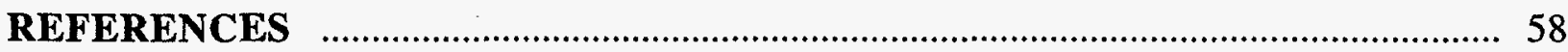

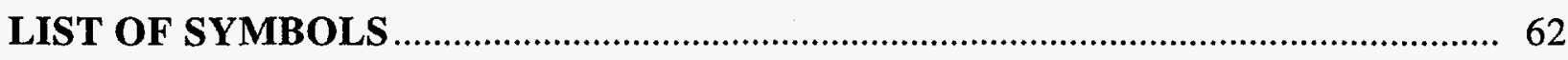

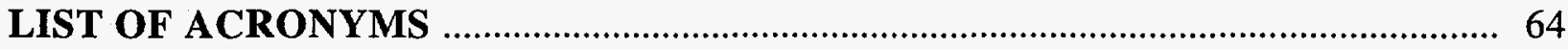




\title{
USE OF ULTRACOLD NEUTRONS FOR CONDENSED-MATTER STUDIES
}

by

\author{
André Michaudon
}

\begin{abstract}
Ultracold neutrons have such low velocities that they are reflected by most materials at all incident angles and can be stored in material bottles for long periods of time during which their intrinsic properties can be studied in great detail. These features have been mainly used for fundamental-physics studies including the detection of a possible neutron electric dipole moment and the precise determination of neutron-decay properties. Ultracold neutrons can also play a role in condensed-matter studies with the help of high-resolution spectrometers that use gravity as a strongly dispersive medium for low-velocity neutrons. Such studies have so far been limited by the low intensity of existing ultracold-neutron sources but could be reconsidered with more intense sources, which are now envisaged. This report provides a broad survey of the properties of ultracold neutrons (including their reflectivity by different types of samples), of ultracold-neutron spectrometers that are compared with other high-resolution instruments, of results obtained in the field of condensed matter with these instruments, and of neutron microscopes. All these subjects are illustrated by numerous examples.
\end{abstract}

\section{INTRODUCTION}

Ultracold neutrons (UCNs) are neutrons of extremely low velocities (below about $6 \mathrm{~m} / \mathrm{s}$ ) that have special properties of great interest in many fundamental-physics studies. Although UCNs have been produced with increasing intensities over the last decades, UCN sources still remain extremely weak compared with sources of higher-energy neutrons. In spite of these low intensities, UCNs have always played an important role in fundamental-physics measurements, including those of the neutron lifetime and electric dipole moment. To a lesser extent, UCNs have also been used in condensed-matter studies, including those of surfaces, magnetic and nonmagnetic films, and slow motions of macromolecules, because UCN reflectivity can be measured with extremely high resolution. 
Studies of the reflection of UCNs by surfaces also proved to be of great practical importance in understanding and reducing the losses in UCN storage, which itself plays a crucial role in fundamental-physics studies. The special properties of UCNs are also used in neutron microscopes.

Condensed-matter studies with UCNs have so far been seriously hampered by the current low intensity of UCN sources. These studies could be reconsidered with new UCN sources that could be more intense than existing sources by one or two orders of magnitude. Before examining the future of condensed-matter studies with more intense UCN sources, which is the main goal of this paper, some basic properties of UCNs are recalled in Sec. II. Because of the interest of UCN reflectivity, some fundamentals of neutron reflectivity are recalled in Sec. III. Quasi-elastic neutron scattering, which could greatly benefit from new UCN instruments, is discussed in Sec. IV. Two existing UCN instruments already used in condensed-matter studies are described in Sec. V. Experimental results obtained with neutron reflectometers, with special emphasis on those obtained with UCN instruments, are given in Sec. VI. Comparison of UCN instruments with other neutron instruments is made in Sec. VII, which also describes the other neutron instruments. The principle and the uses of neutron microscopes are described in Sec. VIII. Finally, conclusions are drawn in Sec. IX.

\section{BASIC PROPERTIES OF ULTRACOLD NEUTRONS}

Slow neutrons are called UCNs when their velocity is below a critical velocity $\mathrm{v}_{\mathrm{c}}$ with the consequence that they are totally reflected by materials at all incident angles. This velocity depends on the nature of the material. For example, $v_{c}$ is equal to $6.9 \mathrm{~m} / \mathrm{s}$ for nickel and to $3.2 \mathrm{~m} / \mathrm{s}$ for aluminum. Total reflection of UCNs comes from the coherent constructive interference of neutron waves elastically scattered by the nuclei in the sample and averaged over a great number of nuclei because of the very long wavelength of UCNs. Under these conditions, the sample behaves as a global effective potential (the Fermi potential) of height $U_{u c n}$ equal to:

$$
\mathrm{U}_{\mathrm{ucn}}=\frac{2 \pi \hbar^{2}}{\mathrm{~m}} \mathrm{Nb}_{\mathrm{coh}}
$$

where $\mathrm{m}$ is the neutron mass, $\mathrm{N}$ is the number of nuclei per unit volume, and $\mathrm{b}_{\text {coh }}$ is the bound coherent scattering length. When $b_{\text {coh }}$ is positive, which is the case for most nuclei, the effective potential is repulsive. For the few cases in which $b_{\text {coh }}$ is negative, the effective potential is negative, and total reflection then occurs at the separation surface from within the sample. The height of the potential $U_{u c n}$ is $252 \mathrm{neV}$ for nickel and $54 \mathrm{neV}$ for aluminum. Equation (1) applies to nonmagnetic samples. The UCN potential for magnetic materials is discussed later in the text. Because the critical velocities and UCN potentials vary from one material to the other, neutrons that are UCNs for some materials may be above UCN energies for others. The neutron energy also varies with the earth's gravitational field; therefore, neutrons can be UCNs at some locations but not at others. In this report, the term UCN is broadly used for neutrons with energies in the UCN domain, i.e., a few tenths of a $\mu \mathrm{eV}$. 
At UCN energies, the neutron-wave properties can be treated with concepts and methods developed for optics. For neutrons, the index of refraction $\mathrm{n}$ of a medium is given by the following formula:

with

$$
\mathrm{n}^{2}=1-\xi,
$$

$$
\xi=\frac{4 \pi}{\mathrm{k}^{2}} \rho \mathrm{b}_{\mathrm{coh}}=\frac{\mathrm{U}_{\mathrm{ucn}}}{\mathrm{E}}=\left(\frac{\lambda}{\lambda_{\mathrm{c}}}\right)^{2} .
$$

In this expression, $\mathrm{k}, \mathrm{E}, \lambda$, and $\lambda_{\mathrm{c}}$ are the wave number, energy, wavelength, and critical wavelength of the neutron, respectively, and $\rho$ is the material density. The critical wavelength is given by the following formula:

$$
\lambda_{\mathrm{c}}=\frac{2 \pi}{\mathrm{k}_{\mathrm{c}}}=\sqrt{\frac{\pi}{\rho \mathrm{b}_{\mathrm{coh}}}},
$$

where $k_{c}$ is the critical wave number. The critical wavelength can be as long as $560 \AA$ (for nickel). For UCNs, wavelengths are longer than $\lambda_{c}$, and the index of refraction given by Eqs. (2) and (3) is therefore imaginary, which is the reason for total UCN reflection.

Above UCN energies, total reflection can also occur for neutrons with incident angles (relative to the surface of the sample) smaller than the critical angle $\theta_{c}$ for which the $\mathrm{z}$-component $\mathrm{k}_{\mathrm{z} 0}$ (along the normal to the surface) of the neutron wave vector is equal to the critical wave number $k_{c}$. In such a case

$$
\sin \theta_{\mathrm{c}}=\frac{\mathrm{k}_{\mathrm{c}}}{\mathrm{k}}=\frac{\lambda}{\lambda_{\mathrm{c}}} .
$$

For cold neutrons of wavelength $10 \AA$, the critical angle on nickel is as small as $1.8 \times 10^{-2}$ radian.

UCNs are very sensitive to the earth's gravitational field. A 1-m change in height causes a change of $100 \mathrm{neV}$ in the neutron potential energy, which is of the same order of magnitude as UCN kinetic energies. UCN trajectories are therefore strongly affected by gravity, which acts as a strongly dispersive medium for UCNs. The influence of gravity on the motion of UCNs is exploited in UCN instruments (see Sec. V).

UCNs can be easily polarized with a high polarization using transmission through thin magnetized iron foils. For magnetized materials, the UCN potential has to take into account the interaction of the neutron magnetic moment $\mu_{\mathbf{n}}$ with the magnetic induction $\mathbf{B}$ in the sample:

$$
\mathrm{U}_{\mathrm{ucn}}=\frac{2 \pi \hbar^{2}}{\mathrm{~m}} \mathrm{Nb}_{\mathrm{coh}}-\mu_{\mathrm{n}} \cdot \mathbf{B} .
$$

The UCN potential then takes one of the two following extreme values, depending on whether $\mu_{\mathbf{n}}$ is antiparallel or parallel to $\mathbf{B}$ :

respectively.

$$
\mathrm{U}_{+}=\frac{2 \pi \hbar^{2}}{\mathrm{~m}} \mathrm{Nb}_{\mathrm{coh}}+\left|\mu_{\mathrm{n}} \mathrm{B}\right| \text {, and } \mathrm{U}_{-}=\frac{2 \pi \hbar^{2}}{\mathrm{~m}} \mathrm{Nb}_{\mathrm{coh}}-\left|\mu_{\mathrm{n}} \mathrm{B}\right| \text {, }
$$


In the energy range $\mathrm{U}_{-}<\mathrm{E}<\mathrm{U}_{+}$( or $\mathrm{E}<\mathrm{U}_{+}$, if $\mathrm{U}_{-}$is negative), only neutrons with their spin antiparallel to $\mathbf{B}$ are transmitted through a thin magnetized iron foil, whereas neutrons with their spin parallel to $\mathbf{B}$ are simply reflected. Because UCN energies are comparable with the magnetic energy $\mu_{\mathrm{n}} \mathrm{B}$ in saturated ferromagnets, UCNs can be polarized through their interaction with ferromagnets. For example, neutron beams with a polarization as high as $95 \%-98 \%$ in the velocity range $4.15 \mathrm{~m} / \mathrm{s}<\mathrm{v}<8.2 \mathrm{~m} / \mathrm{s}$ have thus been obtained (Herdin et al. 1978). Polarized UCNs can be used in the study of magnetic surfaces.

In spite of great progress in UCN production during these last decades, UCN sources are still much weaker than sources of higher-energy neutrons. The most intense source of UCNs is located at the Institut Laue-Langevin (ILL), which is equipped with a high-flux research reactor. Neutrons produced in this reactor are first thermalized in a cold source of liquid deuterium $(T \approx 23 \mathrm{~K})$ and then extracted with a vertical neutron guide before being decelerated to UCN energies by Doppler shifting in a neutron turbine. UCN densities of about $50 \mathrm{UCN} / \mathrm{cm}^{3}$ are thus obtained and stored in a vessel located at the exit of the neutron turbine. The flux at the exit of the neutron turbine is $6 \times 10^{3} \mathrm{~cm}^{-2} \mathrm{~s}^{-1}$ over an area of $8 \mathrm{~cm} \times 15 \mathrm{~cm}$ for UCN velocities below $6.2 \mathrm{~cm} / \mathrm{s}$ (Steyerl 1975, Steyerl et al. 1986). UCN densities higher by one or two orders of magnitude could be expected with the frozen-deuterium technique used with a 1-MW spallation-neutron source, which is proposed by the Los Alamos National Laboratory. Another promising technique is the production of UCNs inside a superfluid-helium bath, where UCN densities higher than existing ones by several orders of magnitude are expected with a 1-MW spallation-neutron source (Golub and Lamoreaux 1994, Lamoreaux 1995a). UCNs could be used either within the He bath itself for fundamental-physics studies or externally by means of a leak through a hole in the wall of the liquid He bath. A UCN source with a high brilliance could then be obtained and used for condensed-matter studies (Lamoreaux 1995b).

More details about the production and properties of UCNs can be found in many other publications (Golub 1996, Golub and Pendlebury 1979, Golub et al. 1979, Golub et al. 1991, Ignatovich 1990, Pendlebury 1993, Steyerl 1983, Steyerl 1989, Steyerl and Malik 1989).

\section{SOME FUNDAMENTALS OF NEUTRON REFLECTIVITY}

\section{A. Introduction}

Neutron reflectivity belongs to the broad field of reflectivity of electromagnetic (EM), acoustic, and particle waves (Lekner 1987, Russell 1990). All these types of reflectivity share many common aspects. Reflectivity of EM and particle waves is the result of a great number of microscopic processes at the atomic or nuclear levels, averaged over macroscopic dimensions and described as macroscopic quantities like the dielectric function $\varepsilon$ for EM waves or an effective potential V in the particle case. Because these microscopic processes depend on the nature of the incident wave, each probe gives access to specific medium properties. For example, the neutron magnetic moment makes it possible to study magnetic properties of surfaces by neutron reflectivity.

Only reflectivity from media with planar stratification is considered here. Such media are simply described with only one spatial variable along the normal to the stratification plane. Neutron reflection at a planar interface between two different media is illustrated in Fig. 1. 


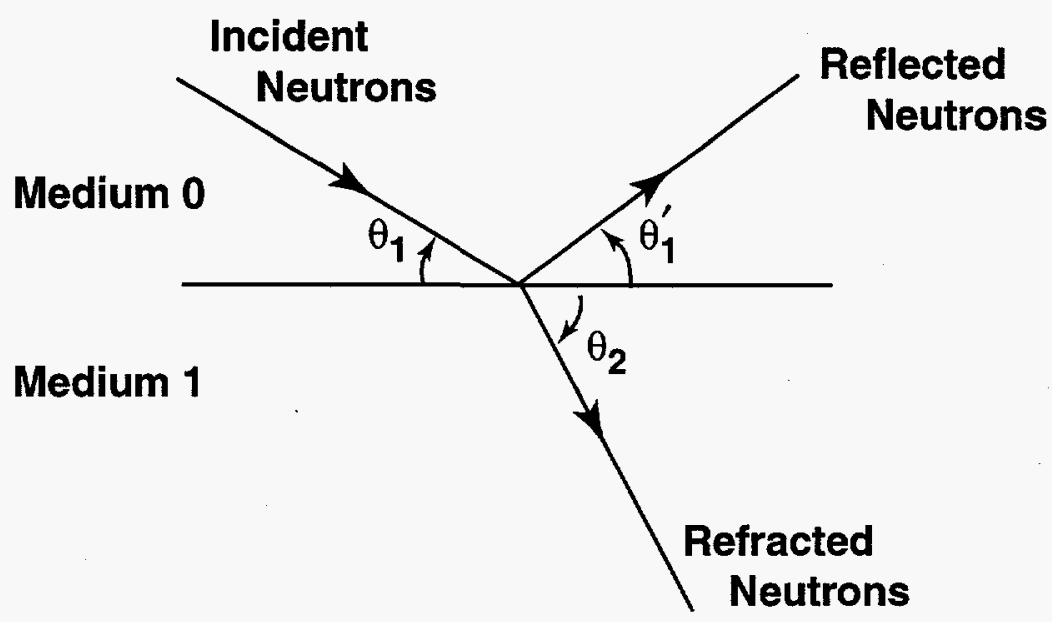

Fig. 1. Reflection of a beam of incident neutrons at a planar interface between media $O$ (usually vacuum) and 1 . The angles of the incident, reflected, and refracted neutrons with the interface are called $\theta_{j}, \theta_{1}^{\prime}$, and $\theta_{2}$, respectively. Sometimes, angles relative to the normal of the surface are also used.

The reflectivity is defined as the ratio of the reflected intensity to the incident intensity and is equal to $R=\left.|r|\right|^{2}$, where $r$ is the reflectivity amplitude.

Neutron reflectivity from a given homogeneous material, with a neutron potential $\mathrm{V}$, is a function only of the component $\mathrm{k}_{\mathrm{z} 0}$ of the neutron wave vector (in medium 0 ) normal to the surface between media 0 and 1 . Total reflection occurs for $\mathrm{k}_{\mathrm{z} 0}$ values below the critical wavelength number $k_{c}$, which depends on the nature of material 1 (when medium 0 is air) but which is typically of the order of $0.01 \AA^{-1}$. In the case of total reflection, the wave vector $\mathrm{k}_{\mathrm{z} 1}$ inside medium 1 is imaginary-the wavefunction inside this medium is therefore evanescent, with a penetration depth that varies between $\lambda_{c} / 2 \pi$ and $\infty$ for neutron energies corresponding to a motion perpendicular to the sample surface and ranging between zero and $\mathrm{U}_{\mathrm{ucn}}$, respectively.

With cold neutrons whose wavelength spectrum peaks at around $\lambda=5 \AA$, wavelength numbers $\mathrm{k}_{\mathrm{z} 0}$ around $\mathrm{k}_{\mathrm{c}}$ can be obtained only at small grazing incident angles of a fraction of a degree. Measurements at such small angles require stringent experimental conditions with tight neutron-beam collimation. These constraints do not exist for UCNs because they are totally reflected at all angles.

When $\mathrm{k}_{\mathrm{z} 0}>\mathrm{k}_{\mathrm{c}}$, the reflected beam is accompanied by a refracted (or transmitted) beam, as illustrated in Fig. 1, and the reflectivity falls off very rapidly with increasing neutron energy. The ratio of the intensity of the transmitted beam to that of the incident beam is $\mathrm{T}$ $=|\mathrm{t}|^{2}$, where $\mathrm{t}$ is the transmission amplitude. Reflectivity and transmission add up to 1 :

$$
\mathrm{R}+\mathrm{T}=1
$$

In addition to the specularly reflected beam, which is symmetric to the incident beam relative to the normal to the surface (Snell's law), diffuse scattering may also be present over a wide range of angles. This type of scattering comes from the incoherent combination of all the waves scattered by the microscopic centers inside the medium. 
Neutron reflectivity from an inhomogeneous material depends on the neutron-potential profile within the medium. Theory aims at obtaining general properties of the reflection and transmission amplitudes and at developing methods for calculating these amplitudes for given neutron-potential profiles. For simple profiles, analytical expressions for $\mathrm{R}$ can be obtained, but for complicated profiles, the reflectivity must be calculated with numerical methods. Some simple cases of reflectivity are presented next for nonmagnetic and magnetic materials (Secs. III.B and III.C, respectively).

\section{B. Neutron Reflectivity from Nonmagnetic Materials}

Because the orientation of the neutron spin cannot be changed by reflectivity from nonmagnetic materials, the expressions derived below are valid for both polarized and unpolarized neutrons.

\section{Reflectivity from an ideal surface}

An ideal surface is a perfect plane that separates two homogeneous media (one of them is usually air) with an abrupt change in the neutron potential across the separation plane. The reflection amplitude is then given by the Fresnel formula:

$$
\mathrm{r}=\frac{\mathrm{k}_{\mathrm{z} 0}-\mathrm{k}_{\mathrm{z} 1}}{\mathrm{k}_{\mathrm{z} 0}+\mathrm{k}_{\mathrm{z} 1}}
$$

with $\mathrm{k}_{\mathrm{z} 1}$ defined as

$$
\mathrm{k}_{\mathrm{z} 1}=\sqrt{\mathrm{k}_{\mathrm{z} 0}^{2}-\mathrm{k}_{\mathrm{c}}^{2}}
$$

Below the critical wave number $\left(\mathrm{k}_{\mathrm{z} 0}<\mathrm{k}_{\mathrm{c}}\right), \mathrm{k}_{\mathrm{z} 1}$ is imaginary, and the reflectivity $\mathrm{R}$ calculated with Eq. (9) is equal to 1, as expected. Above the critical wave number $\left(\mathrm{k}_{\mathrm{z} 0}>\mathrm{k}_{\mathrm{c}}\right)$, the reflectivity $\mathrm{R}$ drops rapidly like $\left[\mathrm{k}_{\mathrm{c}} / \mathrm{k}_{\mathrm{zo}}\right]^{4}$ as a function of $\mathrm{k}_{\mathrm{z} 0}$ for large values of $\mathrm{k}_{\mathrm{z} 0}$ beyond $\left.\mathrm{k}_{\mathrm{c}}\left(\mathrm{k}_{\mathrm{z} 0}\right\rangle \mathrm{k}_{\mathrm{c}}\right)$.

\section{Reflectivity from a plane surface with a soft potential}

The abrupt potential across an ideal surface sometimes needs to be replaced by a "soft potential" to better explain some experimental results. A soft potential may have the Woods-Saxon form, given below, which is widely used in nuclear physics for opticalmodel calculations in spherical coordinates:

$$
\mathrm{V}(\mathrm{z})=\frac{\mathrm{V}_{0}}{1+\exp (\mathrm{z} / \mathrm{d})}
$$

where $d$ is a measure of the thickness of the transition region and $V_{0}$ is a real term that gives the strength of the potential. This potential can be used, for example, for describing surfaces contaminated with physisorbed hydrogenous impurities. 
The reflectivity amplitude caused by potential (Eq. 11) is given by the following expression:

$$
\mathrm{r}=\frac{\sinh \left[\pi \mathrm{d}\left(\mathrm{k}_{\mathrm{z} 0}-\mathrm{k}_{\mathrm{zl}}\right)\right]}{\sinh \left[\pi \mathrm{d}\left(\mathrm{k}_{\mathrm{z} 0}+\mathrm{k}_{\mathrm{zl}}\right)\right]}
$$

where $\mathrm{k}_{\mathrm{zl}}$ is taken far beyond the transition region $\left.\left.(\mathrm{z})\right\rangle \mathrm{d}\right)$. The reflectivity for the soft potential decreases even more rapidly than for an ideal surface with increasing incident wave number $\mathbf{k}_{\mathrm{z} 0}$.

\section{Reflectivity from rough surfaces}

Real surfaces may differ from ideal surfaces because of roughness, i.e., small variations (from a mean value of zero) in height $\zeta_{\mathrm{z}}(\mathrm{w})$ depending on the location $\mathrm{w}=(\mathrm{x}, \mathrm{y})$ on the surface. When these variations are small compared with the incident wavelength, the Rayleigh criterion

$$
2 \pi \zeta_{\mathrm{z}} \cos \theta\left\langle\left\langle\lambda \text { or } \mathrm{k}_{\mathrm{z} 0} \zeta_{\mathrm{z}} \ll<1\right.\right.
$$

for the specular reflection off this surface can be used. For purely random variations in height $\zeta_{z}$, the Gaussian model can be used to calculate the specular reflectivity amplitude $\mathrm{r}\left(\mathrm{k}_{\mathrm{z} 0}\right)$ compared with the amplitude $\mathrm{r}_{0}\left(\mathrm{k}_{\mathrm{z} 0}\right)$ for the ideal case

$$
\mathrm{r}\left(\mathrm{k}_{\mathrm{z} 0}\right)=\mathrm{r}_{0}\left(\mathrm{k}_{\mathrm{z} 0}\right) \exp \left(-2 \mathrm{k}_{\mathrm{z0}} \mathrm{k}_{\mathrm{z} 1}<\zeta_{\mathrm{z}}^{2}>\right)
$$

where $\sqrt{\left\langle\zeta_{\mathrm{z}}^{2}\right\rangle}$ is the standard deviation of the roughness parameter $\zeta_{\mathrm{z}}$.

If the variations in height are not purely random, short-range height-height correlations may exist and may be described in terms of a lateral correlation function $f(\delta)$ defined as

$$
f(\delta)=\lim A^{-1} \int_{A} \zeta_{Z}(w) \cdot \zeta_{Z}(w+\delta) d^{2} w \text { for } A \rightarrow \infty,
$$

where the integral is made over area $\mathrm{A}$.

This lateral correlation function can also be cast into the following Gaussian form:

$$
f(\delta)=\left\langle\zeta_{z}^{2}>\exp \left(-\delta^{2} / 2 \Delta^{2}\right)\right.
$$

where $\Delta$ is the lateral correlation length.

Roughness also gives rise to diffuse scattering, especially at short wavelengths for which the Rayleigh criterion may not be satisfied. Diffuse scattering depends on both the roughness parameter and the lateral correlation length. 


\section{Reflectivity from gratings}

A special case of roughness is that of a periodic corrugation of a sharply defined surface at $\mathrm{z}=\zeta_{\mathrm{z}}(\mathrm{w})$, or $\mathrm{z}=\zeta_{\mathrm{z}}(\mathrm{x})$ in a one-dimensional lateral case. A corrugation with spatial periodicity $\mathrm{d}$ can be expanded into a Fourier series as

$$
\zeta_{z}(x)=\sum_{n=1}^{n=\infty}\left(c_{n} \cos n p x+s_{n} \sin n p x\right)
$$

where the $n$ 's are integers and $p=2 \pi / \mathrm{d}$. The wave function $\psi_{\mathrm{i}}$ of an incident plane wave (perpendicular to the corrugation) at an angle $\theta$ relative to the normal to the averaged surface is given by the following expression:

$$
\psi_{i}=e^{i k_{0}(x \sin \theta+z \cos \theta)}=e^{i\left(K x+k_{20} z\right)}
$$

where $\mathrm{K}=\mathrm{k}_{0} \sin \theta$. Reflected waves include a specularly reflected (of $0^{\text {th }}$ order) wave, which has the following wave function:

$$
\psi_{0}=A_{0} e^{i k_{0}(x \sin \theta-z \cos \theta)}=A_{0} e^{i\left(K x-k_{0} z\right)}
$$

Specular reflection results from constructive interference at an angle $\theta$ between waves scattered at points separated by a distance $d$ on the grating. In specular reflection, there is no path difference between the incident and the reflected waves. But constructive interference (of $\mathrm{n}^{\text {th }}$ order) can also exist at other discrete reflection angles $\theta_{n}$ when the path difference between the incident and scattered waves is equal to $n \lambda$. The angles $\theta_{n}$ obey the following relation:

$$
\mathrm{d}\left(\sin \theta_{\mathrm{n}}-\sin \theta\right)=\mathrm{n} \lambda,
$$

where $\mathrm{n}$ is an integer that can take both positive and negative values. Therefore, the whole spectrum of scattered waves is composed of the specularly reflected wave at an angle $\theta$ and of all the other waves scattered at different discrete angles $\theta_{n}$. The wave function for a given $n \neq 0$ value is given by the following expression:

$$
\psi_{n}=A_{n} e^{i k_{0}\left(x \sin \theta_{n}-z \cos \theta_{n}\right)}=A_{n} e^{i\left(K_{n} x-k_{z 0} z \cos \theta_{n}\right)}
$$

where

$$
K_{n}=k_{0} \sin \theta_{n}=K+p n
$$

The total wave function $\psi$ describing the incident and all scattered waves is given by the following expression:

$$
\psi=\psi_{i}+\psi_{0}+\sum_{n \neq 0} \psi_{n}
$$




\section{Reflectivity from single-layer films}

The reflectivity amplitude $\mathrm{r}$ for a film 1 , of thickness $\mathrm{z}_{1}$, deposited on a thick substrate 2 , is given by the following expression:

$$
r=\frac{r_{1}+r_{2} \exp \left(2 \mathrm{ik}_{\mathrm{zl}} \mathrm{z}_{1}\right)}{1+\mathrm{r}_{1} \mathrm{r}_{2} \exp \left(2 \mathrm{ik}_{\mathrm{zl}} \mathrm{z}_{1}\right)}
$$

where $r_{1}$ is the reflectivity amplitude at the incident surface of the film (as if it were of infinite thickness) and $r_{2}$ is the reflectivity amplitude at the back of the film at the separation surface with the substrate. The reflectivity $R$, which is the square modulus of the reflectivity amplitude $r$, is given by the following formula:

$$
\mathrm{R}=|\mathrm{r}|^{2}=\frac{\mathrm{r}_{1}^{2}+\mathrm{r}_{2}^{2}+2 \mathrm{r}_{1} \mathrm{r}_{2} \cos \left(2 \mathrm{k}_{\mathrm{z} 1} \mathrm{z}_{1}\right)}{1+\mathrm{r}_{1}^{2} \mathrm{r}_{2}^{2}+2 \mathrm{r}_{1} \mathrm{r}_{2} \cos \left(2 \mathrm{k}_{\mathrm{z} 1} \mathrm{z}_{1}\right)}
$$

This expression, which is obtained for real reflectivity amplitudes $r_{1}$ and $r_{2}$, presents an oscillatory behavior as a function of the product $\mathrm{k}_{\mathrm{z} 1} \mathrm{z}_{1}$. If the resolution of the incident neutron beam is broad, Eq. (25) has to be integrated over the corresponding range of $\mathrm{k}_{1}$ values. For a large range of $\mathrm{k}_{1}$ values, this integration yields the following incoherent limit:

$$
\overline{\mathrm{R}}=\frac{\mathrm{r}_{1}^{2}+\mathrm{r}_{2}^{2}-2 \mathrm{r}_{1}^{2} \mathrm{r}_{2}^{2}}{1-\mathrm{r}_{1}^{2} \mathrm{r}_{2}{ }^{2}}
$$

\section{Reflectivity from multilayer surfaces}

A specimen may consist of many layers $i$ of thickness $z_{i}$ on a substrate whose index is $n+1$ (see Fig. 2). At the interface between layers $i$ and $i+1$, a reflectivity amplitude $r_{i}^{\prime}$ is defined in a manner similar to that in Eq. (9), as if the other layers did not exist:

$$
\mathrm{r}_{\mathrm{i}}^{\prime}=\frac{\mathrm{k}_{\mathrm{zi}}-\mathrm{k}_{\mathrm{z}, \mathrm{i}+1}}{\mathrm{k}_{\mathrm{zi}}+\mathrm{k}_{\mathrm{z}, \mathrm{i}+1}} .
$$

In this expression, $\mathrm{k}_{\mathrm{zi}}\left(\mathrm{k}_{\mathrm{z}, \mathrm{i}+1}\right)$ is the $\mathrm{z}$-component of the neutron wave vector in layer $\mathrm{i}(\mathrm{i}+1)$.

\section{Medium 0}

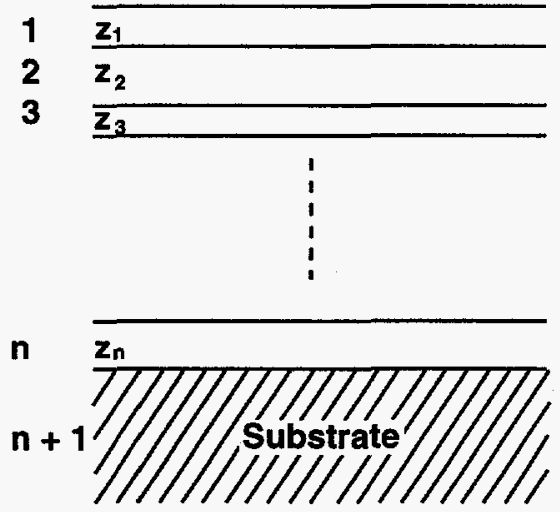

Fig. 2. Diagram of a specimen composed of $n$ layers $i$, each one with a given thickness $z_{i}$, on a substrate whose index is $n+1$. The index 0 corresponds to vacuum. 
The reflectivity amplitude $\mathrm{a}$ into account all layers with in reflectivity amplitude $r_{n-1}$ at $t$ to Eq. (24): $y$ interface between two adjacent layers $i$ and $i+1$ (taking $s$ greater than $(i+1)$ is obtained by first computing the terface between layers $n-1$ and $n$, using a formula similar *as

$$
3=\frac{r_{n-1}^{\prime}+r_{n} \exp \left(2 i k_{z n} z_{n}\right)}{1+r_{n-1}^{\prime} r_{n} \exp \left(2 i k_{z n} z_{n}\right)}
$$

where the amplitude $r_{n-1}^{\prime}$ is $g$

Eq. (27). The amplitude $r_{n-1}$ is then used to calculate the reflectivity amplitude $r_{n-2}$ at th

$$
\frac{r_{n-2}^{\prime}+r_{n-1} \exp \left(2 i k_{z, n-1} z_{n-1}\right)}{+r_{n-2}^{\prime} r_{n-1} \exp \left(2 i k_{z, n-1} z_{n-1}\right)}
$$

Subsequent reflectivity amplit. es are calculated by recursion until the wanted reflectivity amplitude $r_{i}$ is reached. The last amplitude that can be calculated is the total reflectivity amplitude $\mathrm{r}_{0}$ of the specimen.

This method to calculate the reflectivity for a set of discrete layer structures can also be applied to any material whose scattering-length density varies continuously as a function of depth. In such cases, the continuously changing profile can be simulated by a histogram of discrete layers, each one having a constant refractive index.

\section{Quasi-bound macroscopic states of neutrons in matter}

A superposition of several layers of different scattering-length densities can be used to trap neutrons in matter. This structure can be represented by a one-dimensional potential made of square potentials. A double-hump potential can be obtained in this manner-for example, by using a thin film of aluminum, which has a small UCN potential, sandwiched between two layers of copper, which has a much higher $\mathrm{UCN}$ potential (Fig. 3). These layers can be deposited on a substrate of glass or silicon. Macroscopic quasi-bound neutron states can exist in such a $\mathrm{Cu}-\mathrm{Al}-\mathrm{Cu}$ structure and can be detected as resonances in the reflectivity (or transmission) of this structure as a function of incident neutron energy. The total reflectivity $R_{t}$ of this structure is given by the following quantum-mechanical Airy formula:

$$
R_{t}=1-T_{t}=1-\frac{T^{2}}{T^{2}+4 R \sin ^{2}\left(k_{z A l} z_{A l}+\phi\right)},
$$

where $\mathrm{R}(\mathrm{T}$ ) is the reflectivity (transmission) of a single copper layer sandwiched between two semi-infinite media of air and aluminum, $\mathrm{k}_{\mathrm{zAl}}$ is the $\mathrm{z}$-component of the wave vector in aluminum, $\mathrm{z}_{\mathrm{Al}}$ is the thickness of the aluminum layer, and $\phi$ denotes the phase shift of a wave propagating in aluminum when it is reflected on the single copper barrier. Quasi-bound states occur for discrete values of the wave number $\mathrm{k}_{\mathrm{zAl}}$ satisfying the following relation:

$$
\mathrm{k}_{\mathrm{zAl}} \mathrm{Z}_{\mathrm{Al}}+\phi=\mathrm{n} \pi
$$

where $\mathrm{n}$ is the order of the quasi-bound state whose energy is below the copper potential. 

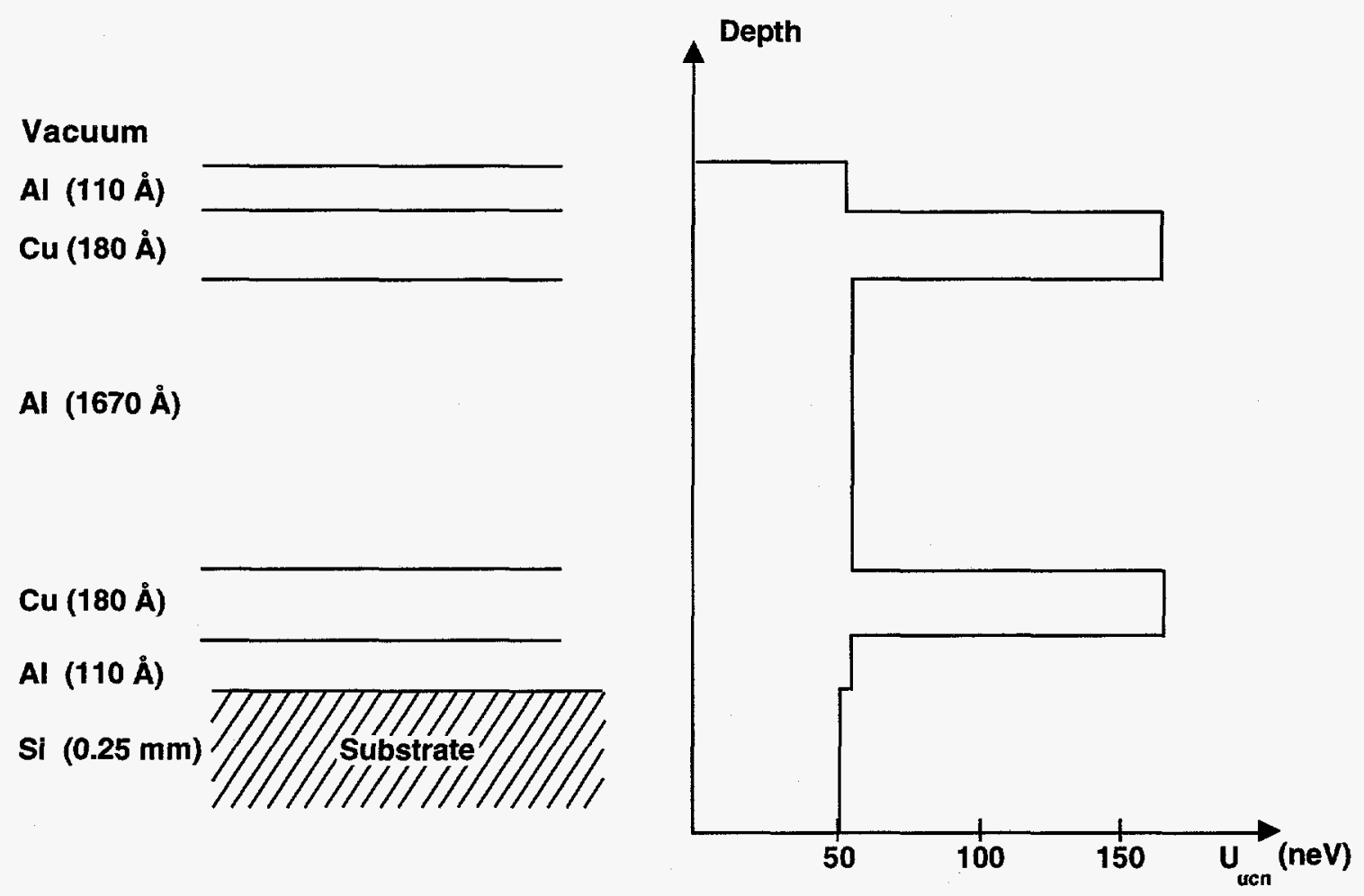

Fig. 3. Diagram of a multilayer sample, which behaves as a double-hump potential for low-energy neutrons and which was used in the study of quasi-bound neutron states (Steinhauser et al. 1980) described in Sec. VI. E.

Only a few quasi-bound states are energetically possible in the $\mathrm{Cu}-\mathrm{Al}-\mathrm{Cu}$ structure below the copper potential. Resonances corresponding to these quasi-bound states appear in the reflectivity (or transmission) curve when the incident neutron energy $\mathrm{E}_{\mathrm{nz}}$ in the perpendicular motion is equal to

$$
\mathrm{E}_{\mathrm{nz}}=\mathrm{U}_{\mathrm{Al}}+\hbar^{2} \mathrm{k}_{\mathrm{zAl}}^{2} / 2 \mathrm{~m},
$$

where $\mathrm{U}_{\mathrm{Al}}$ is the aluminum $\mathrm{UCN}$ potential and $\mathrm{k}_{\mathrm{zAl}}$ takes discrete values according to Eq. (31).

More complicated layer structures can be studied. For example, it is possible to fabricate a structure composed of two identical resonators of the above type coupled by a semitransparent barrier (see Fig. 4). The total reflectivity $R_{t}$ of this structure is given by the following formula:

$$
R_{t}=1-T_{t}=1-\frac{T^{2} T_{1}}{T^{2} T_{1}+\left[(1+R) \sqrt{R_{1}}-2 \sqrt{R} \cos \left(2 k_{z A l} z_{A l}+\phi+\phi_{1}\right)\right]^{2}},
$$

where $R$ and $T\left(R_{1}\right.$ and $\left.T_{1}\right)$ are the reflectivity and transmission, respectively, for the central barrier (lateral barriers) and $\phi$ and $\phi_{1}$ are the phase shifts upon reflection on the central barrier and the lateral barriers, respectively.

Equation (33) gives rise to resonances similar to those of Eq. (30), but for sufficiently strong coupling between the two aluminum wells, each resonance is split into two lines. This type of splitting appears in many other systems of coupled oscillators such as, for example, two coupled pendulums. 


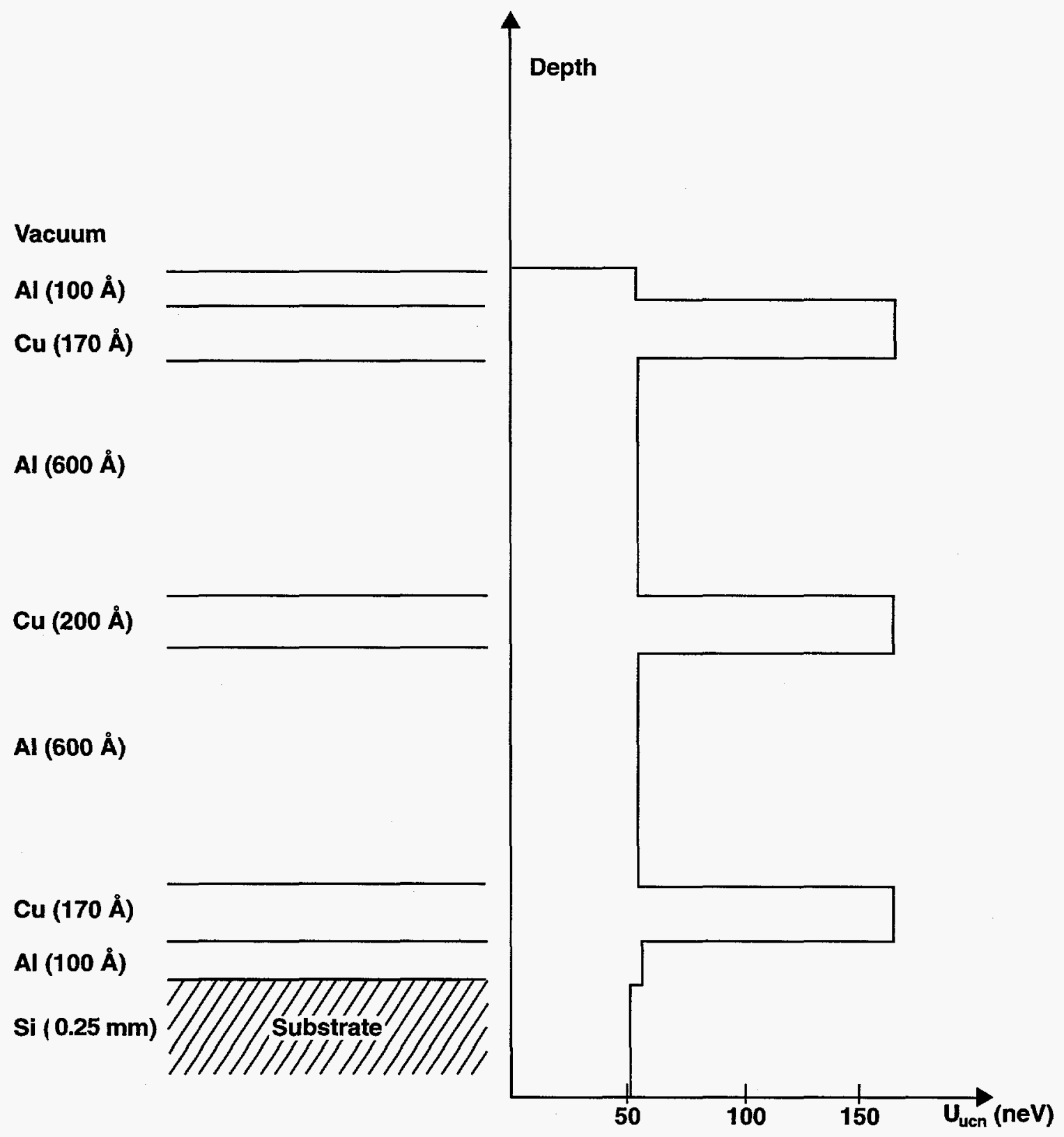

Fig. 4. Diagram of a multilayer system whose potential for low-energy neutrons simulates a coupled-resonator system (Steyerl et al. 1981). This system was used in experiments described in Sec. VI. E.

\section{Neutron Reflectivity from Magnetic Materials}

The interaction potential between neutrons and magnetic materials has the form given by Eq. (6). Because there is no discontinuity in $B_{z}$ (the $z$-component of the magnetic induction B) across the surface of a magnetized material, there is no magnetic effect in neutron reflectivity for samples magnetized perpendicularly to the surface. Neutron reflectivity, however, depends on the surface component of the magnetic induction and on the orientation of this component relative to the neutron spin, which is here supposed to be oriented 


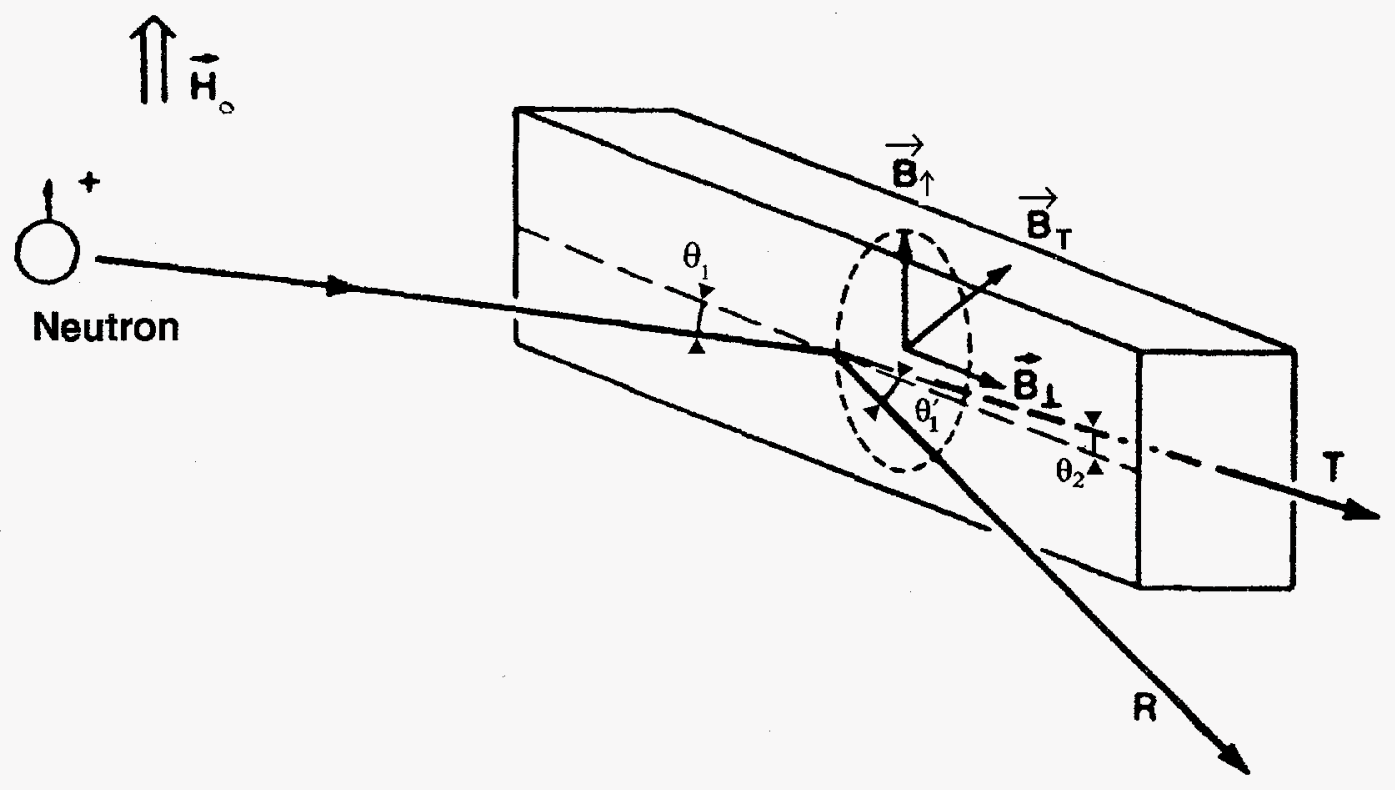

Fig. 5. Scheme of the geometry for a polarized incident neutron beam reflected by a plane magnetized surface. This figure includes the reflected $(R)$ and the refracted $(T)$ neutron beams. Here, the neutron polarization (along an external magnetic field $\boldsymbol{H}_{o}$ ) and the magnetic induction $\boldsymbol{B}$ are restricted to the plane of the surface (Felcher et al. 1987).

parallel to the surface (see Fig. 5). The components of the magnetic induction parallel and normal to the neutron spin (supposed to be vertical here) are called $\mathrm{B}_{\uparrow}$ and $\mathrm{B}_{\perp}$, respectively, with the total magnetic induction $\mathrm{B}_{\mathrm{T}}$ equal to

$$
\mathrm{B}_{\mathrm{T}}^{2}=\mathrm{B}_{\perp}^{2}+\mathrm{B}_{\uparrow}^{2} .
$$

The neutron wave number $\mathrm{k}_{\mathrm{zl}}$ inside the magnetic medium has to take into account the effect of magnetic induction:

$$
\mathrm{k}_{\mathrm{z} 1 \pm}=\sqrt{\mathrm{k}_{\mathrm{z} 0}^{2}-\mathrm{k}_{\mathrm{c} \pm}^{2}},
$$

where

$$
\mathrm{k}_{\mathrm{c} \pm}^{2}=4 \pi\left(\rho \mathrm{b} \pm \mathrm{cB}_{\mathrm{T}}\right) \text {, and } \mathrm{c}=2 \pi \mathrm{m} \mu_{\mathrm{n}} / \mathrm{h}^{2} \text {. }
$$

In these expressions, the indices + or - refer to cases in which the neutron spin is parallel or antiparallel to the magnetic induction vector, respectively. Therefore, the medium behaves as if it had two refractive indices depending on the orientation of the magnetization of the sample relative to the neutron spin.

The intensity of the specularly reflected neutron wave provides information about the magnetic properties of the material under study. The change in neutron polarization, which can also occur during the reflection process, can provide additional information on these magnetic properties. For example, when a polarized neutron beam is reflected by a saturated ferromagnet, there is no change of polarization if the sample magnetization is parallel to the neutron spin, but the neutron polarization vector is rotated when it is not parallel to the sample magnetization. 
The neutron polarization is not changed upon reflection by nonmagnetic materials because there is no spin-dependent term in the interaction potential that can flip the neutron spin. Therefore, polarized UCNs stay polarized when stored in nonmagnetic bottles.

A complete study of the reflectivity of a polarized neutron beam by a magnetic material involves the measurement of both the intensity and the polarization of the reflected neutron beam. Calculations of such reflection properties can become very complicated depending on the case under study. For a general magnetic profile, the reflectivity amplitude cannot be expressed analytically and has to be calculated numerically.

In the simple case of an homogeneous material (i.e., $\rho b$ and $\mathbf{B}$ being constant within the medium) with $\mathrm{B}_{\perp}=0$, the reflectivity amplitude can be simply expressed with the Fresnel formula, Eq. (9), taking into account the possible change in neutron polarization. In this case, the reflectivity amplitude reads

$$
r_{ \pm \pm}=\frac{k_{z}-k_{ \pm}}{k_{z}+k_{ \pm}},
$$

where the index in the first (second) column in the subscripts for $r$ describes the spin state of the incident (reflected) beam. With this particular spin orientation of the neutron spin relative to $B\left(B_{\perp}=0\right)$, there is no spin flip upon neutron reflection $\left(r_{-+}=r_{+}=0\right)$ because the equations for the two neutron spinors are decoupled. For other orientations of the neutron spin relative to $B\left(B_{\perp} \neq 0\right)$, these two equations are coupled making spin flip possible with more complicated expressions for the reflectivity amplitudes.

\section{QUASI-ELASTIC NEUTRON SCATTERING}

\section{A. Introduction}

The above presentation of neutron reflectivity assumes that the atoms in the sample are at rest. This is the static approximation in which the incident neutrons are elastically scattered from the sample atoms (elastic scattering). In real samples, however, atoms are in motion and can exchange energy with the incident neutrons, which can then be scattered at different energies (inelastic scattering). Many dynamical processes can be probed by inelastic neutron scattering. Some of them, like vibrations in a crystal, involve phonons of relatively large energies, which give rise to inelastic neutron peaks clearly separated from the elastic peak. In other cases, however, inelastic scattering occurs with a small neutron energy change, which cannot be seen outside the elastic peak but which can broaden this elastic peak (quasi-elastic scattering).

With UCNs, quasi-elastic scattering can be studied with extremely good resolution for very small values of both the neutron momentum transfer $\hbar \mathrm{Q}$ and the energy exchange $\hbar \omega$. Because the spatial range explored with neutron scattering is of the order of $2 \pi / \mathrm{Q}$, small momentum transfers can probe large distances, as in macromolecules. Small energy transfers $\hbar \omega$ are caused by slow motions associated with long characteristic times $\tau(\omega \tau \leq \pi)$, which are also found in macromolecules. Therefore, quasi-elastic scattering of UCNs looks like a very promising technique to study the dynamics of macromolecules such as various polymers. Because UCNs are available only in very small fluxes, UCN quasi-elastic scattering is restricted to samples that have relatively large surfaces. 
The study of slow dynamics of polymers and of their aggregates is important for a fundamental understanding of polymer shape and interactions. Quasi-elastic light scattering (QELS) of polymer solutions, for example, has been a key element for the study of the size and shape of polymers and polymer aggregates through the determination of the threedimensional diffusion coefficient, $\mathrm{D}$, and the equivalent hydrodynamic radius, $\mathrm{R}_{\mathrm{H}}$. These two quantities are related by the Stokes-Einstein $R_{H}=k T / 6 \pi \eta D$ relationship (where $\eta$ is the solvent viscosity). Typically, QELS makes possible determinations of D over ranges of momentum transfer between $3 \times 10^{-4} \AA^{-1}$ and $5 \times 10^{-3} \AA^{-1}$. With neutrons, the contrast between the target molecule and the solvent can be enhanced by deuteration. Moreover, there is a good match between incident neutron energies and translational energies as well as between the neutron wavelength and the sizes of polymers and polymer aggregates. Neutron-spin-echo (NSE) spectroscopy (described in Sec. VII) has evolved as an important complementary technique for making similar determinations. Typically, NSE measurements can be made with energy transfers from several neV to $\approx 1 \mathrm{meV}$, corresponding to time scales in the range of $1 \mu \mathrm{s}$ to $1 \mathrm{~ns}$, and with momentum transfers down to a few times $0.05 \AA^{-1}$, corresponding to length scales equal to or smaller than $20 \AA$.

There is today a gap in the $\mathrm{Q}$ and $\hbar \omega$ domains of NSE spectroscopy and QELS that could be partially bridged by UCN spectroscopy (Sec. VII). Such a neutron scattering technique could be used to study slow cooperative motions in dense systems and systems where there are strong interactions and/or large structures that are not amenable to light-scattering techniques. Such systems relate to a number of phenomena of practical importance, such as phase separation in blends; sintering processes, such as in drying of coatings; and interactions between polymer melts or solutions and their confining surfaces (Hjelm 1996). The energies and wavelengths of UCNs give the potential of measurements in $Q$ in the $0.01 \AA^{-1}$ range with energy transfers smaller than $1 \mathrm{neV}$ (down to about $0.01 \mathrm{neV}$ ), allowing measurements on time scales larger that $1 \mu$ s with sizes up to $100 \AA$ (Sec. V). The possibility of making measurements in these domains of $Q$ and $\hbar \omega$ with UCNs has already been demonstrated (Sec. VI).

Of special interest for these studies are also biopolymers-proteins and deoxyribonucleic acid (DNA) - which are usually pictured in texts as having a unique and rigid structure. In reality, however, these macromolecules are dynamic systems: they can assume a large number of different structures (conformation substates and fluctuation among these substates). The exploration of the structures and motions of biopolymers, both in equilibrium and nonequilibrium situations, is important for biology and physics. In biology, knowledge of the structures and motions is required for an understanding of the function of biopolymers that is not just descriptive but also predictive. In physics, biomolecules can be considered unique systems that differ from small molecules, liquids, and crystals but are related to glasses and spin glasses. Here the goal is the elucidation of the concepts and laws that govern such complex systems. In particular, knowledge of the energy landscape and the dynamics is desired. The experimental investigation of the structure, energy landscape, and dynamics calls for a wide range of tools, because the number of possible structures is extremely large and motions vary from harmonic to extremely anharmonic and cover times from fs to ks. The energy landscape is rugged and organized hierarchically with a sizable number of tiers. Hence, every conceivable approach, from x-ray scattering to atomic force spectroscopy (AFM), must be involved. Neutrons can play a crucial role in the study of the energy landscape and its dynamics. The time range accessible to UCNs is just right to measure some of the most important motions (Frauenfelder 1996). 
A more quantitative approach to these studies is given below. The general formalism of inelastic-neutron scattering, which includes quasi-elastic scattering, is summarized in Sec. IV.B, and the dynamical processes that can be studied in quasi-elastic scattering are reviewed in Sec. IV.C. More details can be found in Bée (1988).

\section{B. Inelastic-Scattering Formalism}

In contrast to elastic scattering, which provides information about the spatial distribution of the scattering centers supposed to be at rest, inelastic scattering involves analyzing the energy of the scattered neutrons and provides information about the motions of these scattering centers. "Inelastic scattering" applies here to all conditions in which some of the scattered radiation differs in energy from the incident energy $\mathrm{E}_{0}$, however small an amount. With this broad definition, inelastic scattering includes quasi-elastic scattering, in which the energy change of the neutron is small compared with $\mathrm{E}_{0}$. Both coherent and incoherent scattering plays a role in inelastic scattering, as discussed below.

Ignoring the magnetic interaction of the neutron with the sample, the double differential cross section for inelastic-neutron scattering is given by the following relation:

$$
\frac{\mathrm{d} \sigma}{\mathrm{d} \Omega \mathrm{d} \omega}=\frac{\mathrm{k}_{\mathrm{f}}}{\mathrm{k}_{0}}\left[\mathrm{~b}_{\mathrm{coh}}^{2} \mathrm{~S}_{\mathrm{coh}}(\overrightarrow{\mathrm{Q}}, \omega)+\mathrm{b}_{\mathrm{inc}}^{2} \mathrm{~S}_{\mathrm{inc}}(\overrightarrow{\mathrm{Q}}, \omega)\right]
$$

where

- $\mathrm{k}_{0}$ and $\mathrm{k}_{\mathrm{f}}$ are the wave numbers for the incident and scattered neutrons, respectively,

- $b_{\mathrm{coh}}$ and $b_{\text {inc }}$ are the bound coherent and incoherent scattering lengths, respectively,

- $\overrightarrow{\mathrm{Q}}$ and $\omega$ are the momentum and energy transfers (in units of $\hbar$ ), respectively, and

- $\mathrm{S}_{\mathrm{coh}}(\overrightarrow{\mathrm{Q}}, \omega)$ and $\mathrm{S}_{\mathrm{inc}}(\overrightarrow{\mathrm{Q}}, \omega)$ are the dynamic coherent and incoherent structure factors (or scattering laws), respectively.

The quantity in brackets in Eq. (38) is usually called the dynamic structure factor (or scattering law) $S(\vec{Q}, \omega)$, which is simply a weighted sum of the coherent and incoherent dynamic structure factors. Equation (38) strictly applies to monoatomic samples. The formalism for complex compounds is more complicated. Upon integration of $S(\vec{Q}, \omega)$ over $\omega$, one obtains the static structure factor $S(\vec{Q})$ :

$$
\mathrm{S}(\overrightarrow{\mathrm{Q}})=\int_{-\infty}^{+\infty} \mathrm{S}(\overrightarrow{\mathrm{Q}}, \omega) \mathrm{d} \omega \text {. }
$$

This structure factor is also a weighted sum of the components $\operatorname{Scoh}(\overrightarrow{\mathrm{Q}})$ and $\operatorname{Sinc}(\overrightarrow{\mathrm{Q}})$ defined by expressions similar to Eq. (39). The coherent structure factor $S_{c o h}(\vec{Q})$ exhibits an interference pattern, which is used to obtain information about the structure of the sample; the incoherent structure factor $S_{\text {inc }}(\vec{Q})$, which results from a sum of intensities from scattering centers, is Q-independent and just an inconvenient background. Both the coherent and incoherent dynamic structure factors $S_{\operatorname{coh}}(\vec{Q}, \omega)$ and $S_{\text {inc }}(\vec{Q}, \omega)$, however, 
contain valuable information on atomic and molecular motions because there is a quantitative relation between these dynamical structure factors and particle motions.

Let us consider the space-time correlations between nuclei of type $i$ at time $t=0$ and the same nuclei $i$ at time $t \neq 0$ (autocorrelation), on the one hand, and other nuclei $j \neq i$ also at time $t \neq 0$, on the other hand. To this end, let us define the intermediate coherent scattering function $F_{\text {coh }}(\vec{Q}, t)$ as

$$
F_{c o h}(\vec{Q}, t)=\frac{1}{N}\left\langle\sum_{i, j} \exp \left[-i \vec{Q} \cdot \vec{r}_{i}(0)\right] \exp \left[i \vec{Q} \cdot \vec{r}_{j}(t)\right]\right\rangle_{t h},
$$

where $\mathrm{N}$ is the number of nuclei in the sample, the sum is made on all nuclear indices, and the average is made on all the thermodynamic states of the system. For $t=0$, this function reduces itself to the coherent structure factor $S_{\text {coh }}(\vec{Q})$.

$$
\mathrm{F}_{\mathrm{coh}}(\overrightarrow{\mathrm{Q}}, 0)=\mathrm{S}_{\mathrm{coh}}(\overrightarrow{\mathrm{Q}}) \text {. }
$$

For $t \neq 0$, the function $F_{\text {coh }}(\vec{Q}, t)$ gives a statistical description of the way an ensemble of point scatterers evolves in time.

In the same manner, it is possible to define the intermediate incoherent scattering function as

$$
F_{\text {inc }}(\vec{Q}, t)=\left\langle\sum_{i} \exp \left[-i \vec{Q} \cdot \vec{r}_{i}(0)\right] \exp \left[i \vec{Q} \cdot \vec{r}_{i}(t)\right]\right\rangle_{t h}
$$

where the sum in brackets is now on the same nuclear indices $i$. For $t=0$, this function reduces itself to the incoherent structure factor $S_{\text {inc }}(\vec{Q})$, which is actually independent of $Q$ and of no physical interest:

$$
F_{\text {inc }}(\vec{Q}, 0)=S_{\text {inc }}(\vec{Q})
$$

For $t \neq 0$, however, the function $F_{\text {inc }}(\vec{Q}, t)$ gives the correlation in the positions of any same nucleus $i$ at $t=0$ and at any time after $t=0$.

The dynamic structure factors $S_{\text {coh }}(\vec{Q}, \omega)$ and $S_{\text {inc }}(\vec{Q}, \omega)$ are obtained by Fourier transformations with respect to time of the intermediate functions $F_{\text {coh }}(\vec{Q}, t)$ and $F_{\text {inc }}(\vec{Q}, t)$, respectively. The expression for $S_{\text {inc }}(\vec{Q}, \omega)$ reads

$$
S_{\text {inc }}(\vec{Q}, \omega)=\int_{-\infty}^{+\infty} F_{\text {inc }}(\vec{Q}, t) \exp (-\mathrm{i} \omega t) d t,
$$

with a similar expression for $S_{\text {coh }}(\vec{Q}, \omega)$.

The intermediate functions $F$ can also be Fourier-transformed with respect to $\vec{Q}$ to yield the space-time correlation functions $\mathrm{G}(\overrightarrow{\mathrm{r}}, \mathrm{t})$, also known as van Hove correlation functions. The general correlation function $G(\vec{r}, t)$ is obtained as the Fourier transform of the coherent intermediate function $\mathrm{F}_{\text {coh }}(\overrightarrow{\mathrm{Q}}, \mathrm{t})$ :

$$
G(\vec{r}, t)=\int_{-\infty}^{+\infty} F_{c o h}(\vec{Q}, t) \exp (-i \vec{Q} \cdot \vec{r}) d^{3} Q
$$


Formally, $G(\vec{r}, t) d^{3} r$ is the probability of finding particle $i$ at time $t$ in volume element $d^{3} r$ subject to the condition that particle $j$ (which can be the particle $i$ itself or another particle) was at the origin at time $t=0$. It is convenient to separate the function $G(\vec{r}, t)$ into two parts. If nuclei $i$ and $j$ are the same ones, then the part of the function $G(\vec{r}, t)$ that describes the correlation is called the self-correlation function $G_{s}(\vec{r}, t)$. If nuclei $i$ and $j$ are different, then the part of the probability is called the distinct-correlation function $G_{d}(\vec{r}, t)$.

The self-correlation function is the Fourier transform of the incoherent intermediate structure function $\mathrm{F}_{\text {inc }}(\overrightarrow{\mathrm{Q}}, \mathrm{t})$ :

$$
\mathrm{G}_{\mathrm{s}}(\overrightarrow{\mathrm{r}}, \mathrm{t})=\int_{-\infty}^{+\infty} \mathrm{F}_{\mathrm{inc}}(\overrightarrow{\mathrm{Q}}, \mathrm{t}) \exp (-\mathrm{i} \overrightarrow{\mathrm{Q}} \cdot \overrightarrow{\mathrm{r}}) \mathrm{d}^{3} \mathrm{Q} .
$$

Whereas the static incoherent structure factor does not provide any physical information, the dynamic incoherent structure factor can provide information on the correlations in the various positions of any same nucleus as a function of time.

The general correlation function $G(\vec{r}, t)$ is the sum of the self-correlation and the distinct-correlation functions:

$$
G(\vec{r}, t)=G_{d}(\vec{r}, t)+G_{s}(\vec{r}, t)
$$

The functions $G(\vec{r}, t)$ and $G_{s}(\vec{r}, t)$ are the double Fourier transforms of the dynamical coherent and incoherent structure factors $S_{\text {coh }}(\vec{Q}, \omega)$ and $S_{\text {inc }}(\vec{Q}, \omega)$, respectively. These structure factors are usually the quantities directly obtained from measurements. However, some instruments, such as NSE spectrometers, directly give the intermediate structure function (Sec. VII.B).

Examples of atomic and molecular motions in macromolecules and the way they can be detected in dynamical structure factors are given in the next section.

\section{Dynamical Processes Studied in Quasi-Elastic Scattering}

\section{Introduction}

The coherent dynamical structure factor for complex systems is difficult to express and therefore to extract from measurements. For this reason, quasi-elastic neutron-scattering studies have focused on incoherent scattering whose formalism is simpler for complex systems and from which the self-correlation function can be derived. ${ }^{1}$

The motion $\vec{R}(t)$ of a point in a large molecule can result from the combination of vibrational motion $\vec{u}(t)$ relative to a time-averaged position (e.g., as in a crystalline structure), a rotational motion $\vec{\rho}(\mathrm{t})$ relative to the molecular center of mass $(\mathrm{CM})$, and a translational motion of the $\mathrm{CM}$ relative to a laboratory frame. The mathematical description of the overall motion of points in the macromolecule is greatly simplified if these three types of motion are uncoupled, with characteristic times well separated from each other. The scattering law for this combined motion can then be expressed as a convolution product (*) with respect to frequency of the individual scattering laws for vibrational, rotational, and translational motions:

$$
\mathrm{S}_{\mathrm{inc}}(\overrightarrow{\mathrm{Q}}, \omega)=\mathrm{S}_{\mathrm{inc}}^{\mathrm{vibr}}(\overrightarrow{\mathrm{Q}}, \omega) * \mathrm{~S}_{\mathrm{inc}}^{\mathrm{rot}}(\overrightarrow{\mathrm{Q}}, \omega) * \mathrm{~S}_{\mathrm{inc}}^{\mathrm{trans}}(\overrightarrow{\mathrm{Q}}, \omega)
$$

\footnotetext{
${ }^{1}$ Most of this section follows Middendorf (1984).
} 
The intermediate structure function is the straight product of the Fourier transforms of the individual dynamical structure functions for vibration, rotation, and translation. If these three types of motion are coupled, Eq. (48) cannot be applied, and more sophisticated calculations are needed to obtain the global dynamical structure function. Vibrational modes are usually of such amplitudes that their effect on the quasi-elastic peak is reasonably well accounted for by attenuation of this peak by the Debye-Waller factor.

\section{Translational motions}

In a Brownian random-walk path from its initial position at $\mathrm{t}=0$, a particle evolves in a diffusive process that can be described by an "effective" translational diffusion coefficient $\mathrm{D}_{\mathrm{T}}$, which takes into account all the effects that cause diffusion. The mean-square displacement $\left\langle\mathrm{r}^{2}\right\rangle$ of the particle is proportional to $\mathrm{D}_{\mathrm{T}}$ and the time $\mathrm{t}$ :

$$
<\mathrm{r}^{2}>=6 \mathrm{D}_{\mathrm{T}} \mathrm{t} .
$$

For times that are long compared with the average time between two successive collisions, the self-correlation function $G_{s}^{\text {trans }}$ for translational motion obeys Fick's law, which, in spherical coordinates, reads

$$
\frac{\delta}{\delta \mathrm{t}} \mathrm{G}_{\mathrm{s}}^{\text {trans }}=\mathrm{D}_{\mathrm{T}} \mathrm{r}^{-2}\left[\frac{\delta}{\delta \mathrm{r}}\left(\mathrm{r}^{2} \frac{\delta}{\delta \mathrm{r}} \mathrm{G}_{\mathrm{s}}^{\text {trans }}\right)\right] .
$$

When solved, this equation yields the following self-correlation function:

$$
\mathrm{G}_{\mathrm{s}}^{\text {trans }}(\overrightarrow{\mathrm{r}}, \mathrm{t})=\left(4 \pi \mathrm{D}_{\mathrm{T}} \mathrm{t}\right)^{-3 / 2} \exp \left(-\mathrm{r}^{2} / 4 \mathrm{D}_{\mathrm{T}} \mathrm{t}\right)
$$

The scattering law $S_{\text {inc }}^{\text {trans }}(\vec{Q}, \omega)$ for this diffusive process is obtained by a double Fourier transformation of Eq. (51), which leads to the following Lorentzian function:

$$
\mathrm{S}_{\text {inc }}^{\operatorname{trans}}(\overrightarrow{\mathrm{Q}}, \omega)=\frac{1}{\pi} \frac{\mathrm{D}_{\mathrm{T}} \mathrm{Q}^{2}}{\omega^{2}+\mathrm{D}_{\mathrm{T}}{ }^{2} \mathrm{Q}^{4}}
$$

whose total width is

$$
\Delta \mathrm{E}_{\text {inc }}=2 \hbar \mathrm{D}_{\mathrm{T}} \mathrm{Q}^{2} .
$$

Therefore, in the purely Brownian model, there should be a linear dependence of the width of the quasi-elastic peak on $\mathrm{Q}^{2}$. As already stated, this model is valid only for times (or distances) that are long enough compared with the mean time (distance) between two collisions. Consequently, the linear behavior of $\Delta \mathrm{E}_{\text {inc }}$ versus $\mathrm{Q}^{2}$ should be observed only for small $Q$ values. 
Other models, such as those derived from the use of the Langevin equation, also obey a Gaussian approximation of the form given by Eq. (51) but with a width that deviates from the linear time dependence $\mathrm{D}_{\mathrm{T}} \mathrm{t}$. Study of the dependence of $\Delta \mathrm{E}_{\text {inc }}$ on $\mathrm{Q}^{2}$ can therefore provide insight into the nature of diffusive processes.

Translational motions can also occur through jumps between sites, which are shallow potential wells where particles are trapped during times $\tau_{0}$ assumed to be long compared with jump times. Jumps from a given site to its $n_{s}$ nearest-neighbor sites may be described by a set of vectors $\vec{d}_{n}$ extending from the initial site to its $n_{s}$ sites. Assuming isotropy, the incoherent scattering function for this process is also a Lorentzian of width

$$
\Delta E_{\text {inc }}(\vec{Q})=\frac{h}{\pi n_{s} \tau_{0}} \sum_{n=1}^{n_{s}}\left[1-\exp \left(-i \vec{Q} \cdot \overrightarrow{d_{n}}\right)\right]
$$

For jumps involving large $n_{s}$ values and random vectors of the same length $d_{0}$, Eq. (54) reduces to

$$
\Delta \mathrm{E}_{\mathrm{inc}}=\frac{\mathrm{h}}{\pi \tau_{0}}\left(1-\frac{\sin \mathrm{Qd}_{0}}{\mathrm{Qd}_{0}}\right),
$$

which presents an oscillatory behavior typical of jump diffusion models. In the limit $\mathrm{Q} \rightarrow$ 0 , the process appears Brownian-like with a width

$$
\Delta \mathrm{E}_{\text {inc }}=\frac{\mathrm{h}}{\pi}\left(\frac{\mathrm{d}_{0}^{2}}{6 \tau_{0}}\right) \mathrm{Q}^{2},
$$

which is expressed in terms of the microscopic quantities $\tau_{0}$ and $\mathrm{d}_{0}$.

It is also possible to combine several types of translational motions among themselves with possible vibrations in the sites of the jump models.

\section{Rotations}

In the diffusive motions considered above, the particle drifts through a Brownian random-walk path away from its initial position and gradually forgets its initial parameters. The self-correlation function of Eq. (51) actually goes to zero when the time goes to infinity. This behavior is in contrast with that of rotations in which the particle is confined to the same volume, called $\mathrm{V}_{\infty}$, at any time during the motion. The rotational correlation function $G_{s}^{\text {rot }}(\vec{r}, t)$ therefore approaches a limit called $G_{s \infty}^{\text {rot }}(\vec{r})$ when the time goes to infinity. The rotational self-correlation function can then be split off as the sum of two components:

$$
G_{s}^{\text {rot }}(\vec{r}, t)=G_{s \infty}^{\text {rot }}(\vec{r})+G_{s}^{\text {rot' }}(\vec{r}, t)
$$

which, after double Fourier transformation, leads to the following rotational incoherent structure factor, which is also the sum of two components:

$$
\mathrm{S}_{\mathrm{inc}}^{\mathrm{rot}}(\overrightarrow{\mathrm{Q}}, \omega)=A_{0}(\overrightarrow{\mathrm{Q}}) \delta(\omega)+\mathrm{S}_{\text {inc }}^{\mathrm{rot}^{\prime}}(\overrightarrow{\mathrm{Q}}, \omega)
$$

One component is the sharp elastic contribution given by the Dirac function, which is called the elastic incoherent structure factor (EISF). In the ideal case of isotropic Brownian 
rotational motion, the volume $\mathrm{V}_{\infty}$ is occupied with equal probability for long times. But more complex modes are possible in which some parts of $\mathrm{V}_{\infty}$ will be occupied with greater probability than others.

\section{ULTRACOLD-NEUTRON GRAVITY INSTRUMENTS}

\section{A. Introduction}

Because the earth's gravitational potential strongly influences the motion of UCNs, gravity can be used to produce monochromatic UCN beams and to analyze the energy of UCNs scattered from samples. Two UCN gravity instruments have so far been used in condensed-matter studies. One of them, called a UCN gravity diffractometer, uses directly the energy gained or lost in the vertical motion of UCNs (Scheckenhofer and Steyerl 1981). The other one, called a UCN spectrometer or more specifically NESSIE (for Neutronen Schwerkraft Spektrometrie in German or neutron gravity spectrometry in English), uses gravity as a strongly dispersive medium for the motion of UCNs (Steyerl et al. 1983). These instruments were first installed at the UCN source of the Forschungs Reactor Munich (FRM), which has a thermal-neutron flux of $10^{13} \mathrm{n} / \mathrm{cm}^{2} \mathrm{~s}$, and subsequently moved to the more intense UCN source of the ILL with the same neutron turbine to slow cold neutrons down to UCN energies.

\section{B. The UCN Gravity Diffractometer}

A schematic view of a recent version of the UCN gravity diffractometer is given in Fig. 6 (Steyerl et al. 1992). A horizontal UCN beam with a velocity $v_{x}=(4 \pm 0.2) \mathrm{m} / \mathrm{s}$ is fed from the neutron turbine into the entrance beam system having a vertical slit width $\Delta \mathrm{h}=0.4 \mathrm{~cm}$.

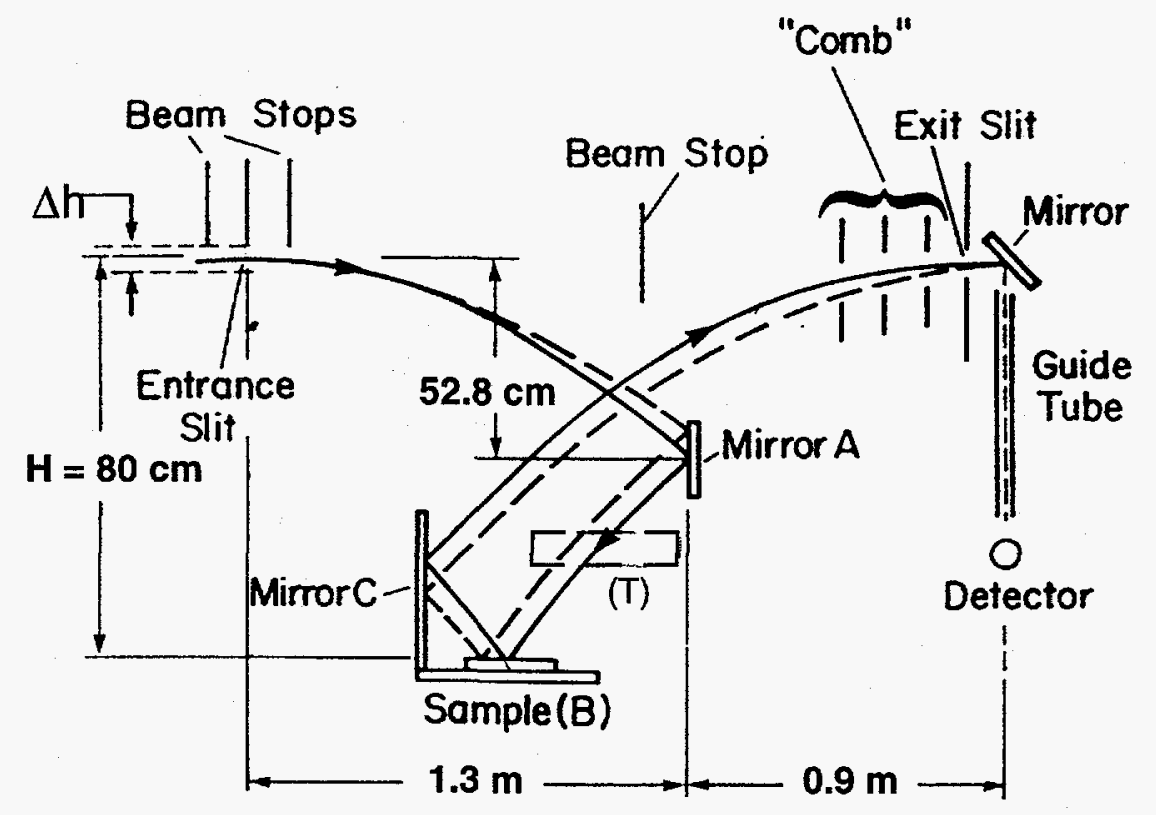

Fig. 6. Schematic view of the UCN gravity diffractometer. This instrument is mainly used in the reflection mode but can also be used in the transmission mode by replacing sample $B$ with a mirror and placing the sample at position $(T)$. 
This slit defines the initial resolution $\Delta \mathrm{E}_{\mathrm{ucn}}=0.4 \mathrm{neV}$ of the $\mathrm{UCN}$ beam before it falls under gravity onto a horizontal sample (or mirror) $\mathrm{B}$, where it is reflected ufwards to an exit slit whose width matches that of the entrance slit. During their motion between the entrance and exit slits, UCNs are reflected and focused on the exit slit by many mirrors. Mirrors A and C are shown in Fig. 6, but there are about 10 to 20 other lateral mirrors (not shown in this figure), which are vertical and perpendicular to mirrors $\mathrm{A}$ and $\mathrm{C}$. After the exit slit, the UCNs are reflected downwards by an inclined mirror into a guide tube, where they are accelerated by gravity to be counted in $\mathrm{aF}_{3}$ detector.

Stringent requirements on the alignment and surface quality of all mirrors placed on the UCN trajectories are mandatory for good operation of the UCN gravity diffractometer. The energy spectrum of the UCN beam at the exit of the diffractometer can be analyzed by vertically moving the exit slit. The overall resolution $\Delta \mathrm{E}_{\mathrm{ucn}}$ depends critically on the alignment of the mirrors of the diffractometer. This resolution is typically around $1 \mathrm{neV}$ and can be as low as $\Delta \mathrm{E}_{\mathrm{ucn}}=0.51 \mathrm{neV}$, as illustrated in Fig. 7. Quasi-elastic scattering can be detected by broadening of the reflectivity line with a sensitivity as good as a few $0.01 \mathrm{neV}$ (Steyerl et al. 1992) but over a limited range of the momentum transfer, from 0.01 to $0.015 \AA^{-1}$. The vertical energy of the neutrons incident on sample (or mirror) B typically varies from 65 to $120 \mathrm{neV}$ as a result of changing the height of sample $B$. This energy can exceed the UCN potential for some materials, which can also be studied with refracted neutrons transmitted through the sample placed between mirrors A and B. The sample size is typically $5 \mathrm{~cm} \times 15 \mathrm{~cm}$.

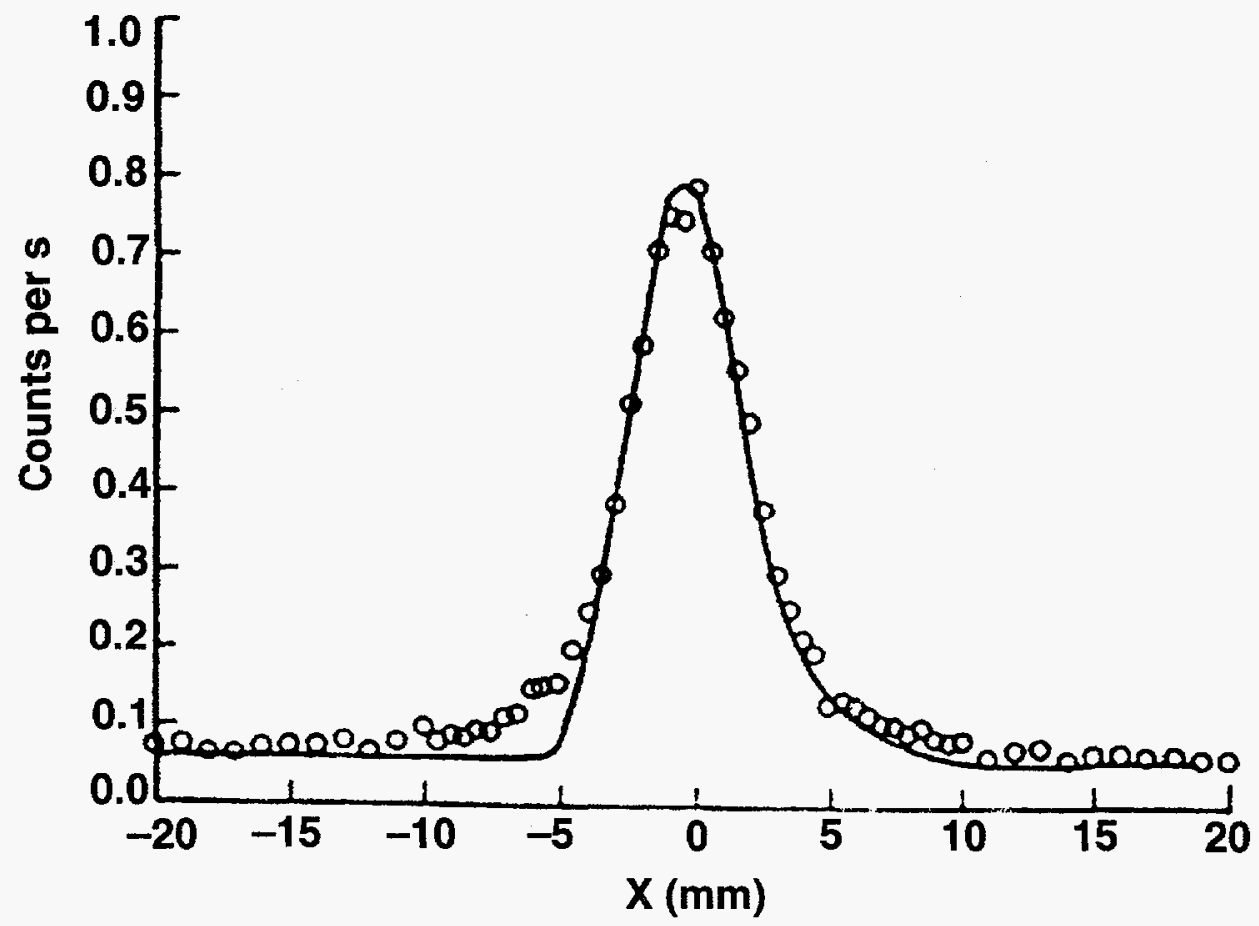

Fig. 7. Illustration of the energy resolution achieved with the UCN gravity diffractometer shown in Fig. 6. The count rate (circles) is plotted as a function of vertical position $x$ of the exit slit. This experimental beam profile is fitted by a Monte Carlo calculation (solid curve). Both measurements and calculations agree with a resolution width-full width at half maximum (FWHM) - of $5.1 \mathrm{~mm}$, i.e., $0.51 \mathrm{neV}$ (Steyerl et al. 1992). 
With perfect total UCN reflection at all mirrors and ideal geometry, the count rate of the gravity diffractometer at the ILL is 10 counts/s with $0.4-\mathrm{cm}$ slits (Steyerl et al. 1992). This count rate may be decreased by imperfections in the alignment and contamination of the mirrors. This count rate is about 250 times that obtained at the FRM ( 140 counts/h) with 2 -cm slits and a UCN source about $10^{4}$ less intense than that of the ILL. Using the same resolution both at the ILL and at the FRM, the count rate would be about $1.25 \times 10^{3}$ higher at the ILL, still less than the $10^{4}$ difference in the intensity of the UCN sources at both places. Reflectivities with count rates of 10 counts/h were measured at the FRM (Scheckenhofer and Steyerl 1977). Assuming that the same sensitivity could be achieved at the ILL, reflectivities down to about $10^{-4}$ could be measured with the UCN gravity diffractometer installed at the ILL and set to a resolution of $2 \mathrm{neV}$. A further improvement by two orders of magnitude in the intensity of UCN sources would push the sensitivity of reflectivity measurements down to $10^{-6}$. However, the limited $\mathrm{Q}$ range $\left(10^{-2} \AA^{-1} \leq \mathrm{Q} \leq\right.$ $1.5 \times 10^{-2} \AA^{-1}$ ) of the UCN gravity diffractometer would severely restrict the interest of such small-reflectivity measurements because there is a close relationship between the reflectivity, the $\mathrm{Q}$ value, and the energy of the diffractometer. For example, the $\mathrm{Q}$ and energy values are related by the following simple expression:

$$
\mathrm{Q}=2 \mathrm{k}_{\mathrm{zo}}=0.0014 \sqrt{\mathrm{E}_{\mathrm{dif}}(\mathrm{neV})}\left(\AA^{-1}\right),
$$

where $E_{\text {dif }}$ is the neutron-fall energy of the diffractometer. The present maximum energy of the diffractometer is $120 \mathrm{neV}$, which restricts $\mathrm{Q}$ measurements to values below $0.015 \AA^{-1}$. Even doubling the height of the instrument, which is quite big already, would only increase the maximum accessible $\mathrm{Q}$ value by $40 \%$. The dependence of the reflectivity on $\mathrm{Q}$ (or $\mathrm{k}_{\mathrm{zo}}$ ) above the critical energy depends on the sample, but a rough estimate can be derived from the Fresnel formula of Eq. (9):

$$
\mathrm{R}=\left(\frac{\mathrm{k}_{\mathrm{c}}}{\mathrm{k}_{\mathrm{zo}}}\right)^{4}=\left(\frac{\mathrm{U}_{\mathrm{ucn}}}{\mathrm{E}_{\mathrm{Dif}}}\right)^{2} .
$$

Therefore, reflectivities of $10^{-4}$ already attainable today would be of interest only for samples having a very small positive UCN potential (about $1 \mathrm{neV}$ ) or a negative UCN potential.

\section{The UCN Gravity Spectrometer}

The other instrument, NESSIE, uses the strongly dispersive properties of the gravitational field for UCNs in a geometry shown in Fig. 8 (Steyerl 1978). A neutron of total kinetic energy $E_{0}$ emitted at an angle $\alpha$ relative to the horizontal direction follows a parabolic trajectory in the gravitational field until this particle reaches the point of impact. The horizontal distance $\mathrm{R}$ traveled by the particle during its flight is given by the formula

$$
\mathrm{R}=\frac{4 \mathrm{E}_{0}}{\mathrm{mg}} \cos ^{2} \alpha(\tan \alpha-\tan \beta),
$$




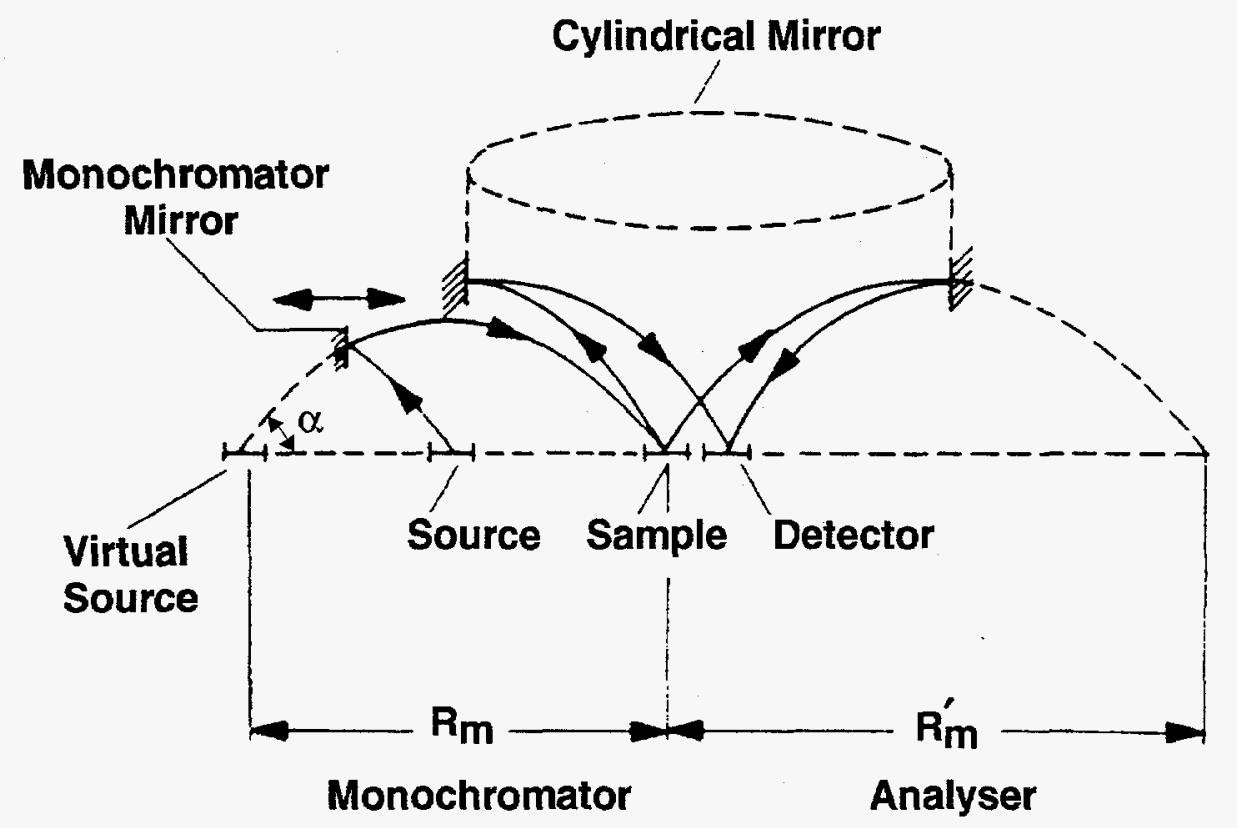

Fig. 8. Principle of the gravity spectrometer NESSIE (Steyerl 1978).

where $\beta$ is the angle that the straight line joining the launching point and the point of impact makes with the horizontal direction and $\mathrm{g}$ is the earth's gravitational field. For a given energy $E_{0}$, the maximum reach $R_{m}$ will be achieved for an angle of emission $\alpha_{s}$ given by the formula

$$
\alpha_{\mathrm{s}}=\tan ^{-1}\left(\frac{1+\sin \beta}{\cos \beta}\right)
$$

for which the value of $R_{m}$ is

$$
R_{m}=R\left(\alpha_{s}\right)=\frac{2 E_{0} \cos \beta}{m g(1+\sin \beta)}
$$

Note that this value of $R_{m}$ is stationary with respect to $\alpha$, which makes it possible to focus at this distance a fairly divergent beam of monochromatic neutrons. The divergence $\Delta \alpha$ of the incident beam is, however, limited by the second-order correction to a value given by

$$
\Delta \alpha \leq\left(\Delta \mathrm{R} / 2 \mathrm{R}_{\mathrm{m}}\right)^{1 / 2}
$$

where $\Delta R$ is the uncertainty in $R_{m}$ caused by the sizes of the source and of the sample.

For small values of $\beta$, the sensitivity of the maximum reach $R_{m}$ to the incident energy $E_{0}$ is obtained by differentiating Eq. (63), which gives the relation

$$
\frac{\Delta \mathrm{R}_{\mathrm{m}}}{\Delta \mathrm{E}_{0}} \approx 2 \mathrm{~cm} / \mathrm{neV}
$$

independent of incident energy. This high value illustrates the strongly dispersive nature of the earth's gravitational field for neutrons and demonstrates that the maximum-reach method can yield a very good neutron-energy resolution. 
Although the maximum-reach method could be used, in principle, for any neutron energy, constraints on the sizes of spectrometers restrict it to very low energy neutrons such as UCNs. The distance $R_{m}$ is proportional to $E_{0}$ as can be seen from Eq. (61) and is already equal to $8 \mathrm{~m}$ for $\mathrm{E}_{0}=0.4 \mu \mathrm{eV}$. Moreover, the spectrometer requires large-angle deflection mirrors, which can be realized only for very low energy neutrons.

NESSIE is designed to focus on the detector a fairly large angular range of the neutrons scattered by the sample. This is achieved by the use of a cylindrical mirror of slightly elliptical shape, with one focal point at the sample and the other at the detector. The neutron energy after scattering is determined by the horizontal flight $R_{m}^{\prime}$.

In its actual design (Fig. 9), the incident energy at the sample can be varied from $0.4 \mu \mathrm{eV}$ to $0.6 \mu \mathrm{eV}$, which are energies substantially above those of the gravity diffractometer. NESSIE is optimized for a sample size of $5 \times 5 \mathrm{~cm}^{2}$. Experiments have, however, been carried out with larger samples measuring $5 \times 15 \mathrm{~cm}^{2}$ (Pfeiffer et al. 1988) but likely incurred a loss of resolution, as a consequence of Eq. (65). The momentum transfer is limited to a narrow range between 0.02 and $0.03 \AA^{-1}$, about a factor of 2 above that of the gravity diffractometer. The resolution is typically around $16 \mathrm{neV}$, much broader than that of the gravity diffractometer (Fig. 10). A line broadening of $2 \mathrm{neV}$ can be observed in quasielastic scattering experiments (Pfeiffer et al. 1988). The flux of monochromated neutrons at the sample position is about $2.5 \mathrm{n} / \mathrm{cm}^{2} / \mathrm{min}$, nearly independent of incident neutron energy, with the FRM source of UCNs (Steyerl et al. 1983). The count rate is very low: about 24 counts $/ \mathrm{h}$ for a $5 \times 15 \mathrm{~cm}^{2}$ sample (Steyerl et al. 1983, Pfeiffer et al. 1988).

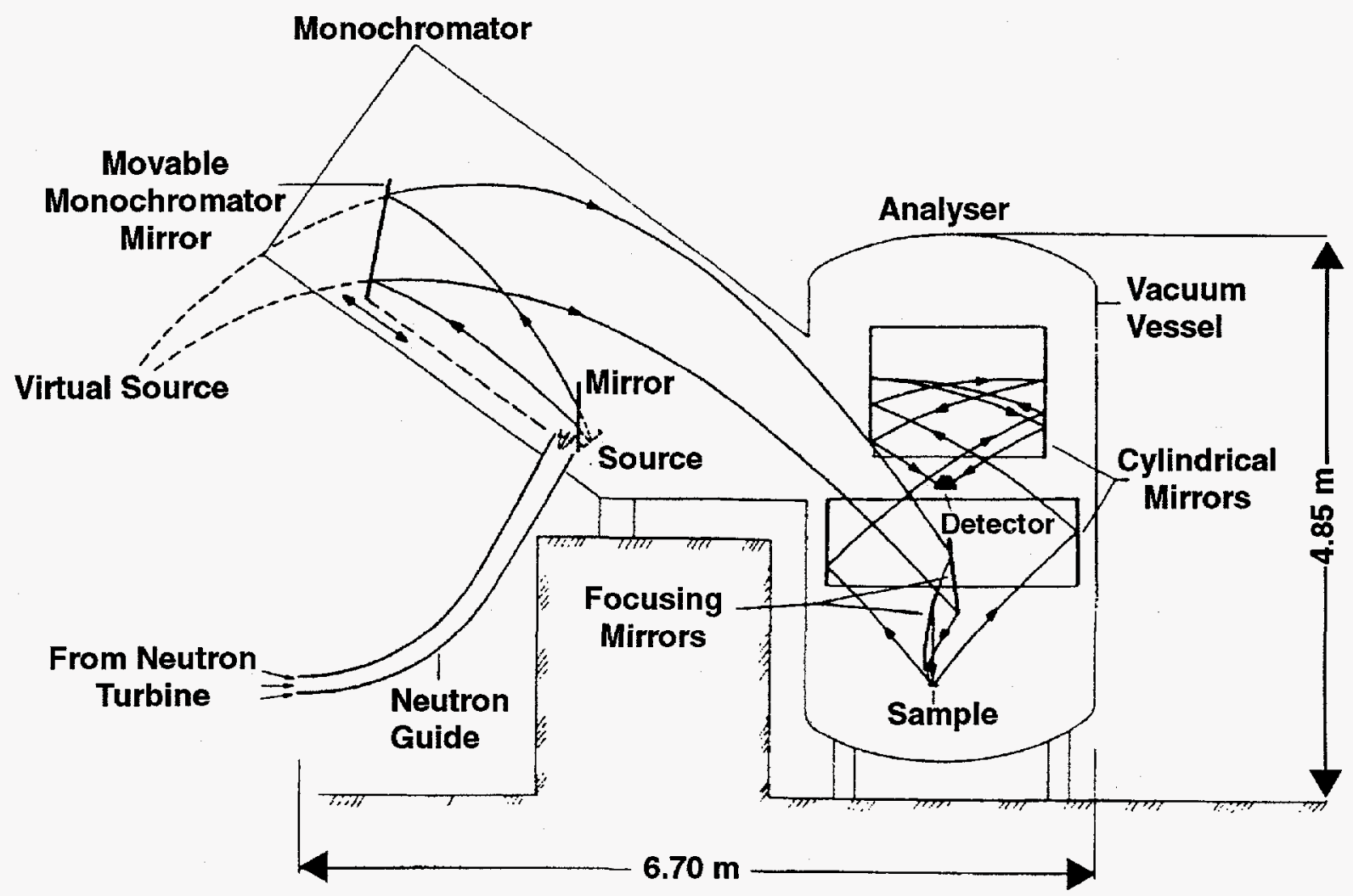

Fig. 9. Sketch of the arrangement of the gravity spectrometer NESSIE at its design stage (Steyerl 1978). This instrument is described in more detail elsewhere (Steyerl et al. 1983). 


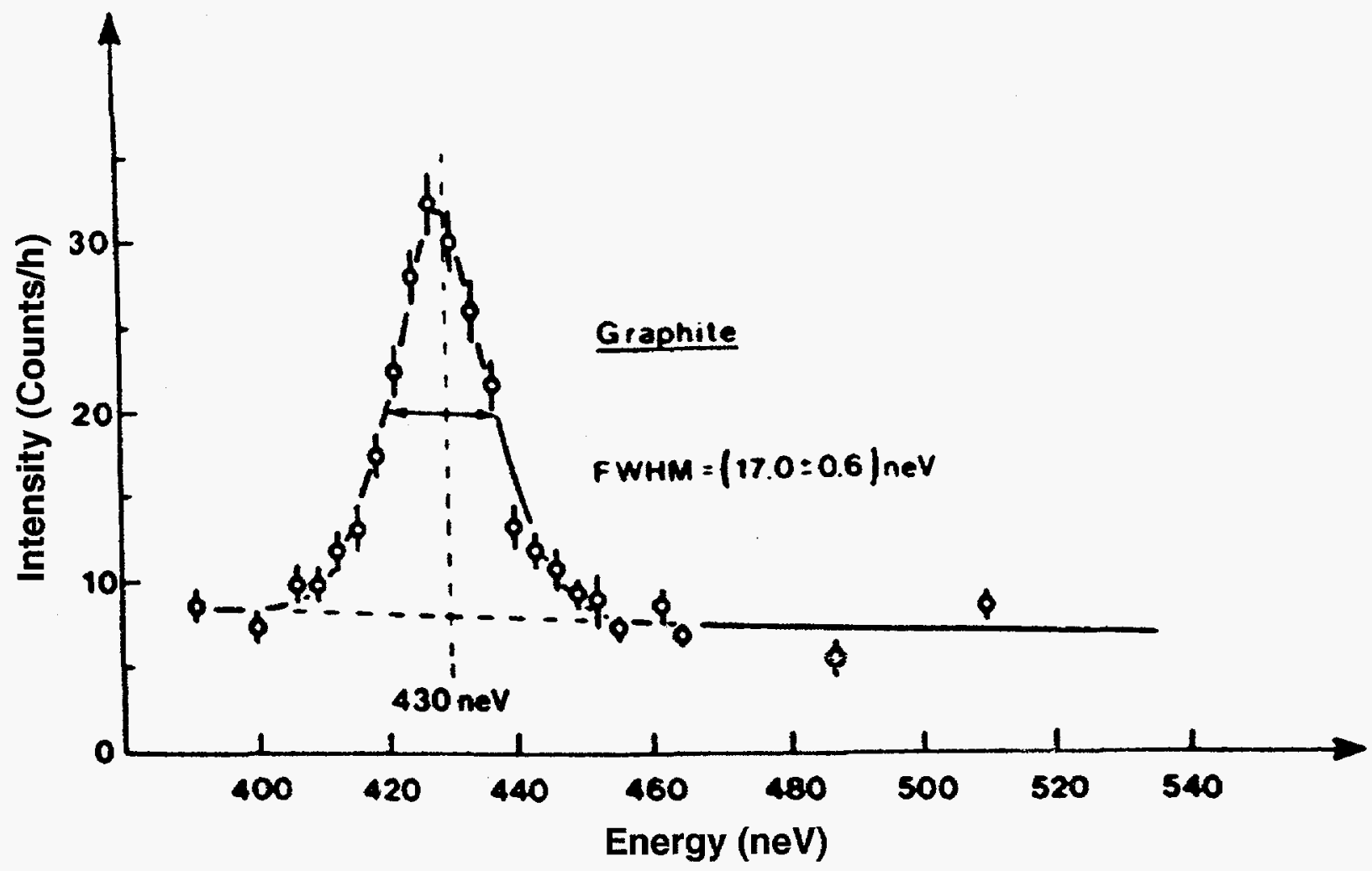

Fig. 10. The measured energy curve of NESSIE, as obtained by elastic scattering from a graphite sample. The resolution curve is plotted as a function of incident-neutron energy and is centered on the energy of $430 \mathrm{neV}$. The FWHM of the resolution curve is (17.0 \pm 0.6) neV (Steyerl et al. 1983).

\section{Conclusions}

These two instruments have been used in many condensed-matter studies, most of the time in the reflectivity mode described above but sometimes in the transmission mode. Note that NESSIE selects neutrons according to their total kinetic energy $E_{0}$, in contrast to the gravity diffractometer, in which the vertical neutron energy (the energy for vertical motion) is defined. For both instruments, there is, however, a close relation between the total and the vertical energies for each setting and mode of operation.

Both instruments can make measurements only in a limited range of small $Q$ values. Illustrations of these studies are given in Sec. VI. The gravity diffractometer seems better suited than NESSIE for most future studies. This diffractometer provides a better resolution ( $0.5 \mathrm{neV}$ FWHM compared with $16 \mathrm{neV}$ for NESSIE) and a higher count rate (140 counts/h compared with 24 counts/h for NESSIE at the FRM). It is also easier to operate than NESSIE, which is a huge and complex instrument. The relatively high count rate of the gravity diffractometer compared with that of NESSIE comes from the fact that the diffractometer can be used with a comparatively wide interval of horizontal neutron velocities and therefore with a significant beam divergence without deterioration of resolution. 


\section{SOME EXPERIMENTAL RESULTS IN NEUTRON REFLECTOMETRY}

Some experimental results obtained in neutron reflectometry with several instruments are illustrated below. In some cases, these results are supplemented by transmission measurements.

\section{A. Reflection from a Smooth Planar Surface}

The use of the gravity diffractometer to measure neutron reflectivity from planar surfaces at neutron energies across the critical energy is illustrated in the two following experiments carried out at the FRM.

In the first experiment, neutrons are incident on the planar surface of liquid lead (Fig. 11). Above the critical energy, the reflectivity drops sharply as a function of neutron vertical energy, which is determined by the height of neutron fall. This rapid decrease in reflectivity is well reproduced with the Fresnel formula, Eq. (9), used with an abrupt potential. This measurement yields a very precise value of the critical energy, from which an equally precise value (to $0.02 \%$ ) of the lead neutron scattering length can be obtained (Koester et al. 1986). This excellent precision explains why this instrument was widely used for very precise scattering-length measurements.

In the second experiment, neutrons are incident on a low-alumina boron-free float glass mirror (Fig. 12). The resolution is $3 \mathrm{neV}$, and the count rate at total reflection is about 145 counts/h (background subtracted). A steep edge is observed at the critical height of $93.6 \mathrm{~cm}$, but the drop in reflectivity beyond the critical energy is even more rapid than predicted by an abrupt potential. The data are, however, well reproduced with a soft potential of the type given by Eq. (11) using a thickness $d=73 \pm 3 \AA$ for the transition region. This result can be interpreted as having been caused by a physisorbed hydrogenous surface layer whose concentration in $\mathrm{H}_{2} \mathrm{O}$ decreases with increasing depth (Scheckenhofer and Steyerl 1977). The amount of hydrogen derived from these data is the right amount to explain the relatively high UCN losses observed in bottled UCNs in early ILL measurements.

\section{B. Effect of Roughness on the Reflectivity from and Transmission through Planar Surfaces}

A simple example of the influence of roughness on reflectivity is given in Fig. 13, where cold neutrons are incident at a grazing angle, therefore with a very small vertical energy, on a rough 2-mm-thick lead film that was grown on an optically flat silicon support (Felcher et al. 1987). The incident cold neutrons are emitted from a cold moderator of solid methane (at $\mathrm{T}=10 \mathrm{~K}$ ) that is irradiated by the fast neutrons produced with the intense pulsed neutron source (IPNS) of the Argonne National Laboratory (ANL). The fixed angle of incidence is $\theta_{1}=0.355^{\circ} \pm 0.020^{\circ}$, and the wavelength reflectivity dependence is measured by the time-of-flight (TOF) method. The reflectivity at short wavelengths drops more drastically than that given by the Fresnel formula for a smooth surface. Modifying the Fresnel formula by a roughness attenuation factor given by Eq. (14) gives, however, a good fit to the data with a surface roughness $\sqrt{\left\langle\zeta_{z}^{2}\right\rangle}=65 \AA$ (Felcher et al. 1987). 


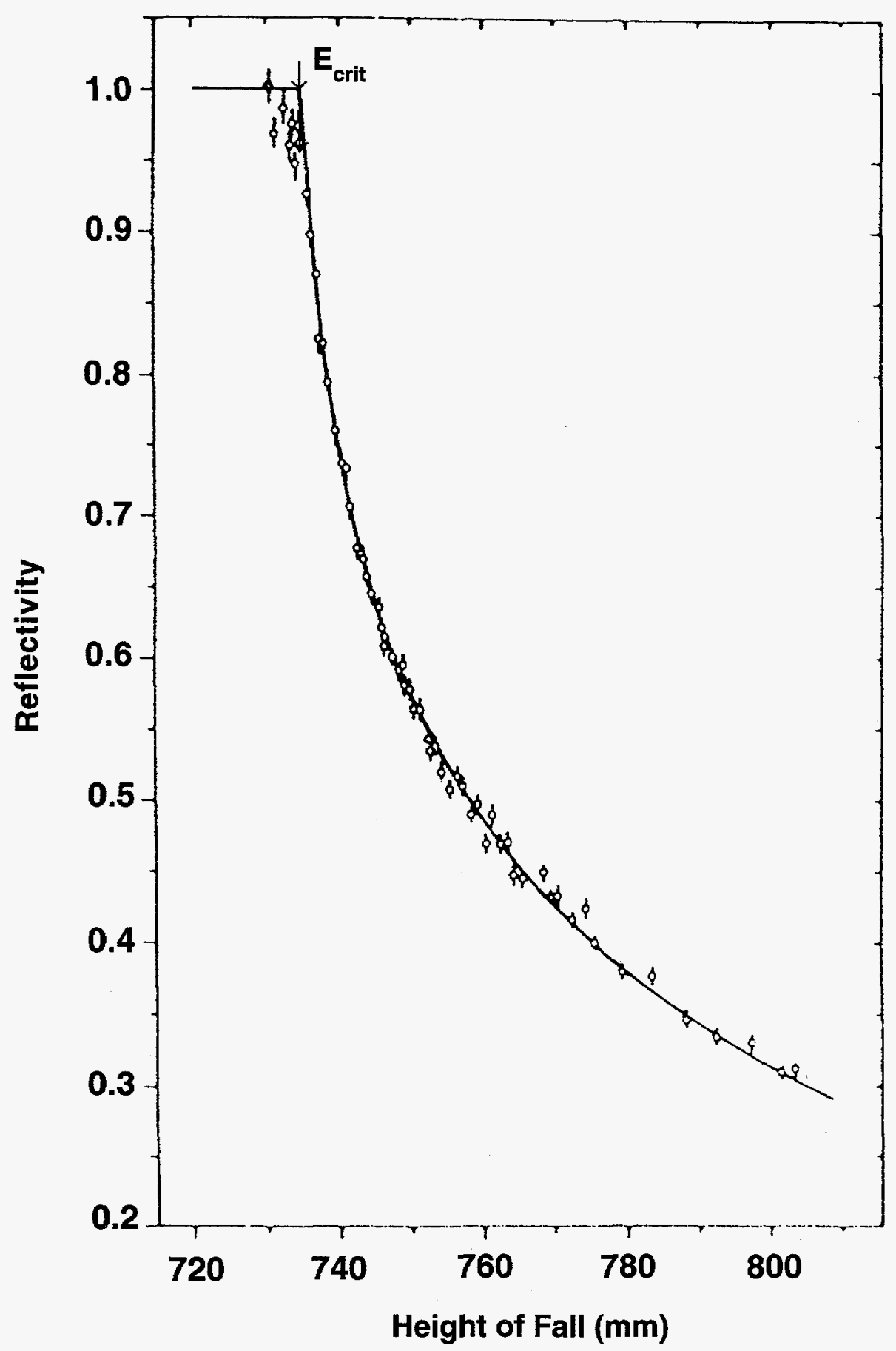

Fig. 11. Reflectivity of liquid lead as a function of the height of fall of the incident neutrons, as measured with the neutron gravity diffractometer (Koester et al. 1986.) Solid circles are experimental data, and the solid line is a fit to these data calculated for an undisturbed surface of the liquid lead. This figure is reprinted with kind permission from Elsevier Science - NL, Sara Burgerhartstraat 25, 1055 KV Amsterdam, The Netherlands. 


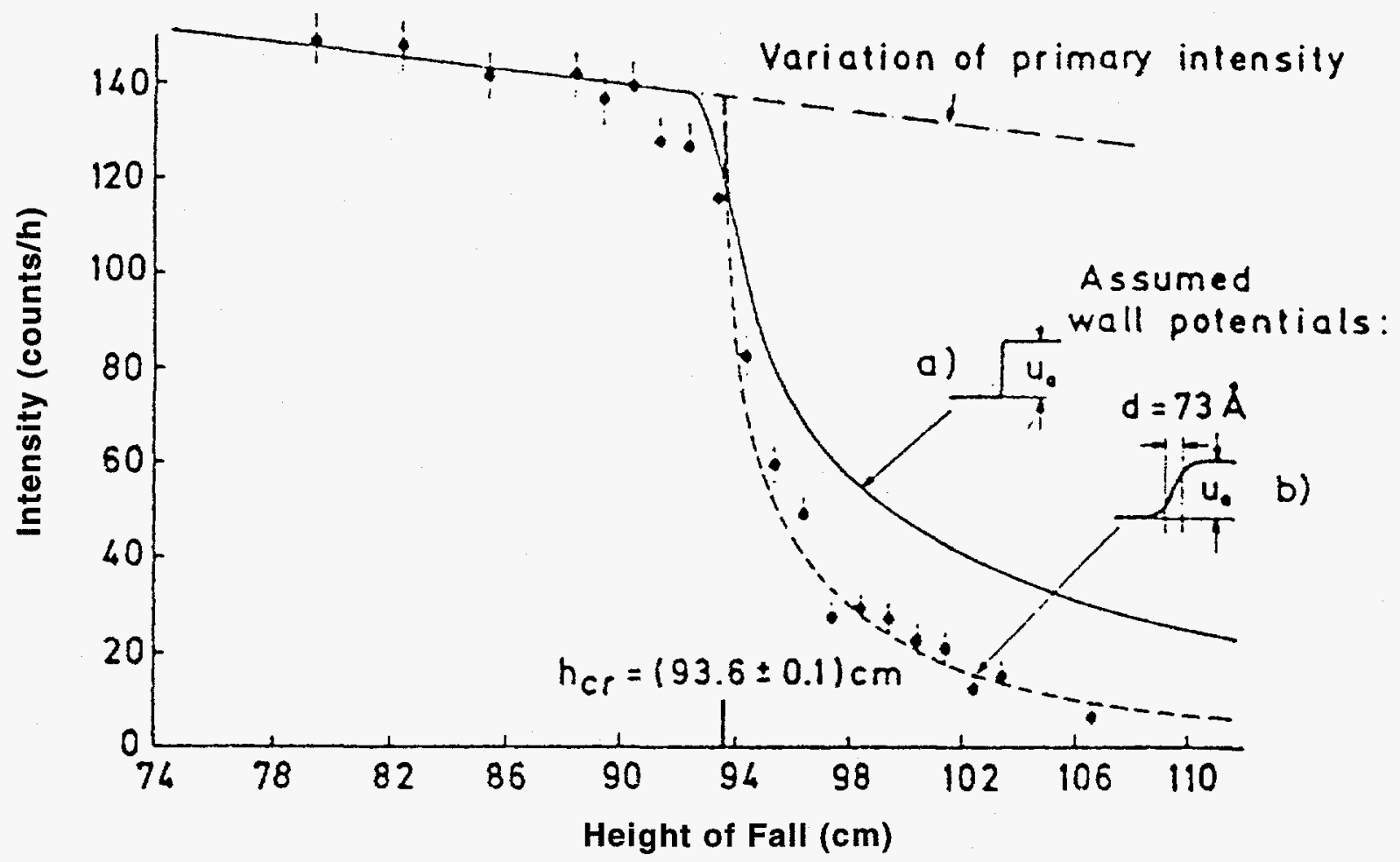

Fig. 12. Intensity of neutrons reflected from a glass mirror measured with the UCN gravity diffractometer as a function of the height of fall of the incident neutrons. The experimental data (solid circles) are compared with theoretical calculations by means of a wall scattering potential, which is either a sharp step function [solid line (a)] or a smoothed step function [dashed line (b)] (Scheckenhofer and Steyerl 1977).

The influence of roughness can also be seen in the transmission of very cold neutrons through samples, as illustrated in Fig. 14, which shows the total cross section $\sigma_{\mathrm{T}}$ obtained from neutron transmission through a 0.12 -mm-thick polycrystalline copper sheet. The measurements were carried out with the source of very cold neutrons of the FRM using the TOF method (Steyerl 1972a, Steyerl 1972b). These measurements were made at room temperature, before and after mechanical polishing of the surfaces, for incident neutron velocities $\mathrm{v}_{0}$ above the critical velocity for copper (which is $\mathrm{v}_{\mathrm{c}}=5.62 \mathrm{~m} / \mathrm{s}$ ). Because of roughness, a fraction of the incident neutrons is scattered away from the incident beam, causing a loss in the transmitted neutron beam and resulting in an apparent increase in $\sigma_{\mathrm{T}}$. In the absence of roughness, the measurement should give the true cross section at velocity $v^{\prime}$ of the neutrons inside the sample, which has to take into account the loss of kinetic energy of the incident neutrons upon crossing the Fermi potential at the front surface of the sample $\left(\mathrm{v}^{\prime}=\sqrt{\mathrm{v}_{0}^{2}-\mathrm{v}_{\mathrm{c}}^{2}}\right)$. This is actually observed with the sample that has polished surfaces, which yields a measured $\sigma_{\mathrm{T}}$ obeying a $1 / \mathrm{v}^{\prime}$ behavior, as expected. But the measurements with a sample having rough surfaces yield a $\sigma_{\mathrm{T}}$ which deviates from the $1 / \mathrm{v}^{\prime}$ law. The apparent increase in $\sigma_{\mathrm{T}}$ that is observed with rough surfaces can be accounted for with a Gaussian lateral correlation function given by Eq. (16) and the following parameters: $\sqrt{\left\langle\zeta_{z}^{2}\right\rangle}=100 \AA$, and $\Delta=500 \AA$. 


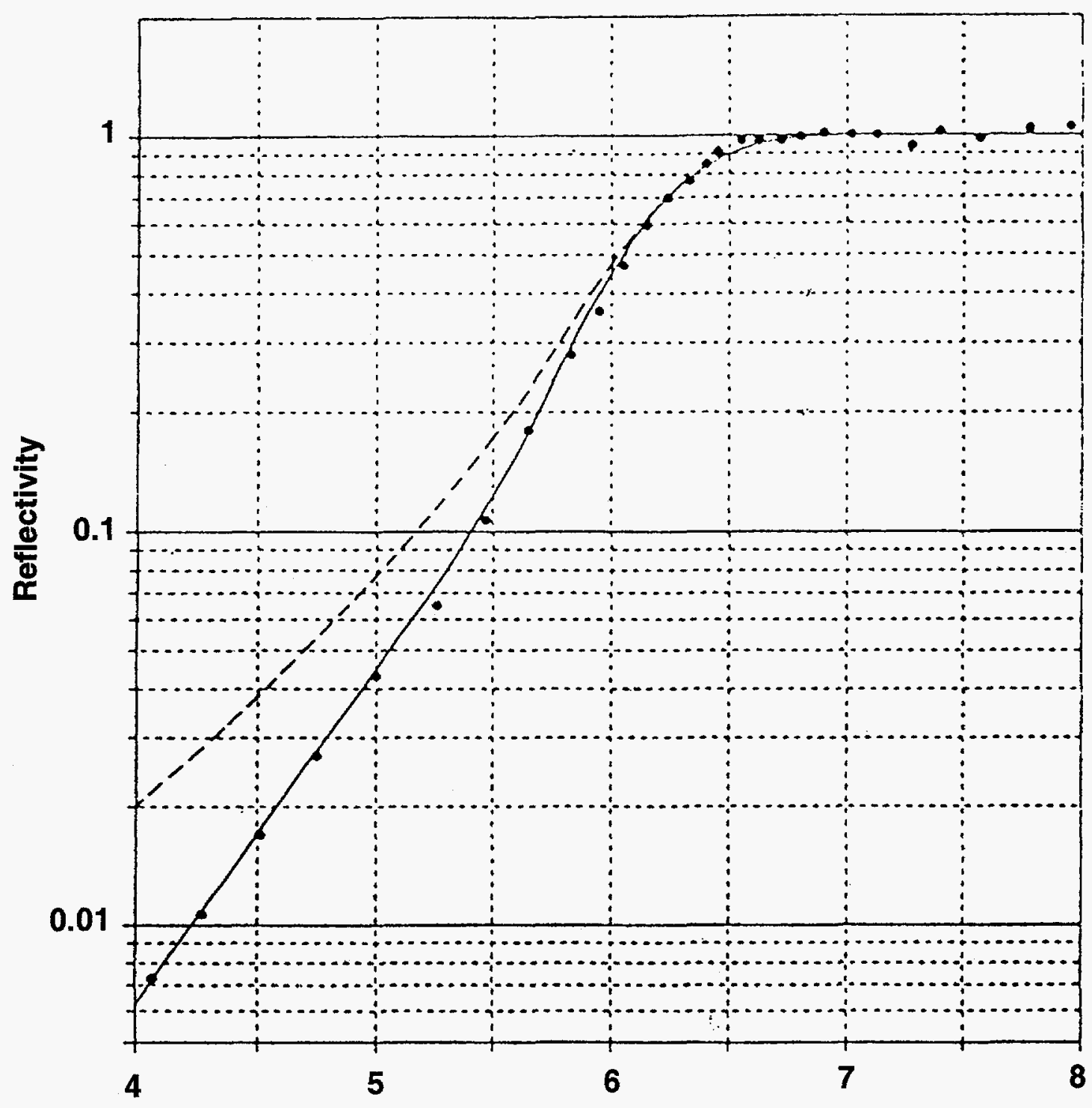

Neutron Wavelength (Angstroms)

Fig. 13. Measured neutron reflectivity of a lead film deposited on a silicon support as a function of the incident neutron wavelength (solid circles). The measurements were made with the grazing-angle neutron reflectometer installed at the IPNS. The calculated reflectivity for an ideal plane surface is represented by a dashed line. A surface roughness of $65 \AA$ has been added in the calculations shown on the solid line (Felcher et al. 1987). 


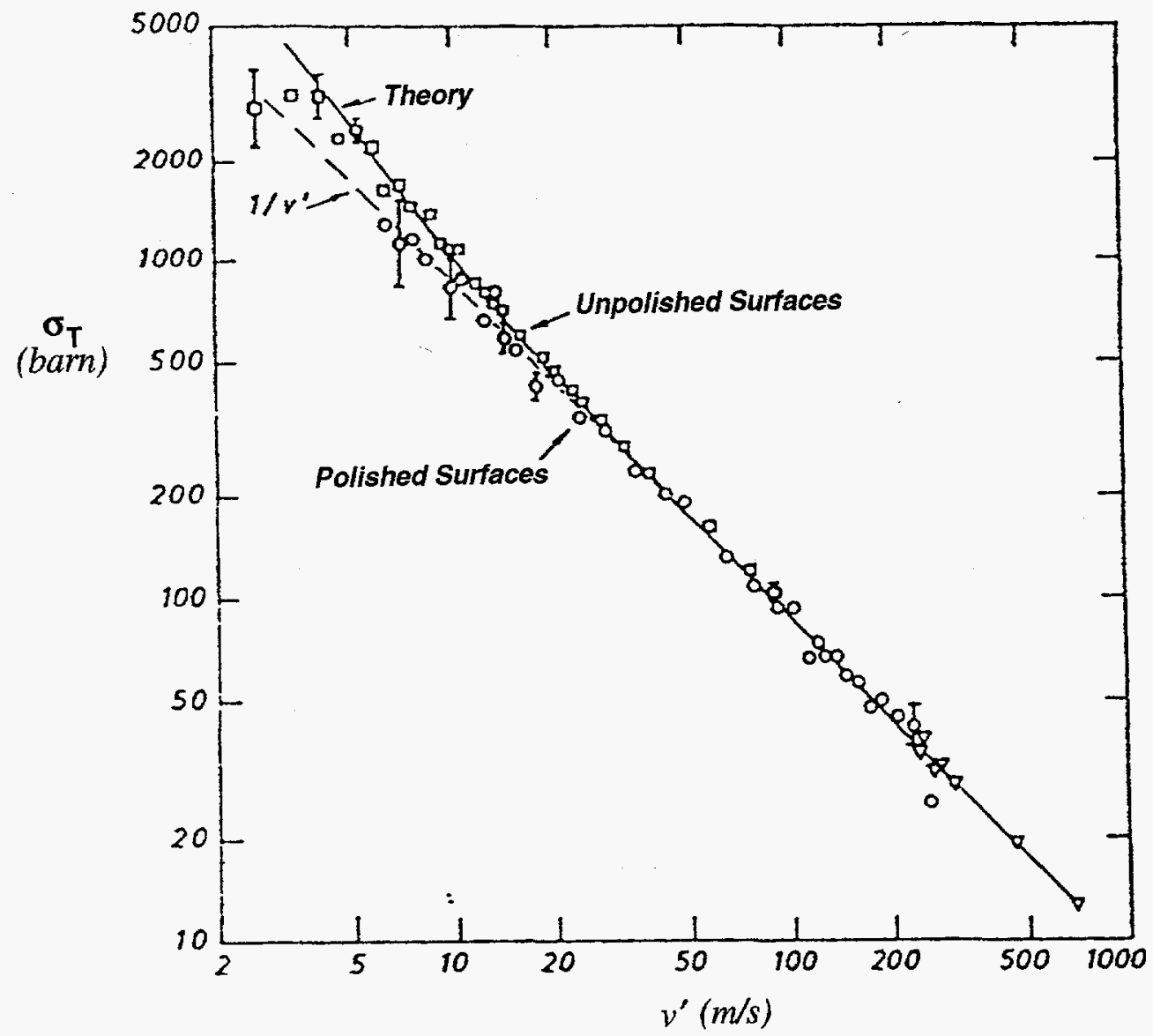

Fig. 14. Measurements of the total cross section $\sigma_{T}$ of copper at room temperature for very cold neutrons as a function of the neutron velocity $v^{\prime}$ within the sample. The data points were obtained by measuring the transmission of a copper foil before and after mechanical polishing (open squares and open circles, respectively), using the neutron TOF method (Steyerl 1969, Steyerl $1972 \mathrm{~b}$ ). The cross section obtained from the polished sample obeys the expected $1 / v^{\prime}$ dependence (dashed curve) whereas the data obtained from the unpolished copper sample deviate from this behavior because a significant fraction of the incident neutrons are scattered away by surface roughness. The experimental data obtained before polishing are in good agreement with calculations (solid curve) that take into account roughness with parameters given in the text (Steyerl 1972a).

\section{Reflectivity from Gratings}

Gratings were studied at high resolution with the neutron gravity diffractometer installed at the ILL (Steyerl et al. 1992).

Reflection from gratings was studied in the $0^{\text {th }}$-order diffraction mode, which gives the classical specular reflection, and the first-order modes $(n=+1$ and -1$)$. For the study of the $0^{\text {th }}$-order mode, the sample with gratings was located at the horizontal position $B$ (see Fig. 6). Specular reflectivity was studied as a function of $\mathrm{k}_{\mathrm{z} 0}$ by varying the vertical position of the sample and as a function of $\mathrm{k}_{\mathrm{x} 0}$ (the horizontal component of the wave number) by varying the position of mirror A (Fig. 6) and adjusting the positions of the other mirrors. For the study of reflections of order $n=-1,0,+1$, the sample with gratings was placed at the location of mirror A (Fig. 6). 
The variation of the measured specular reflectivity from a grating as a function of $\mathrm{k}_{\mathrm{z} 0}$ and $\mathrm{k}_{\mathrm{x} 0}$ is plotted in Fig. 15, which includes the groove profile of this grating. The data are compared with calculations made assuming the Rayleigh criterion, Eq. (13), and using the Rayleigh approximation, which supposes that waves diffracted from elevated parts of the groove surface into the groove depth can be neglected. The dependence of the reflectivity on $d / D$ (not shown on the diagram) is very strong. A reasonably good fit to the data is obtained for $d / D=0.22$. For this value of $d / D$, the dependence of the reflectivity on $k_{z 0}$ is fairly strong whereas that on $\mathrm{k}_{\mathrm{xo}}$ is very weak.

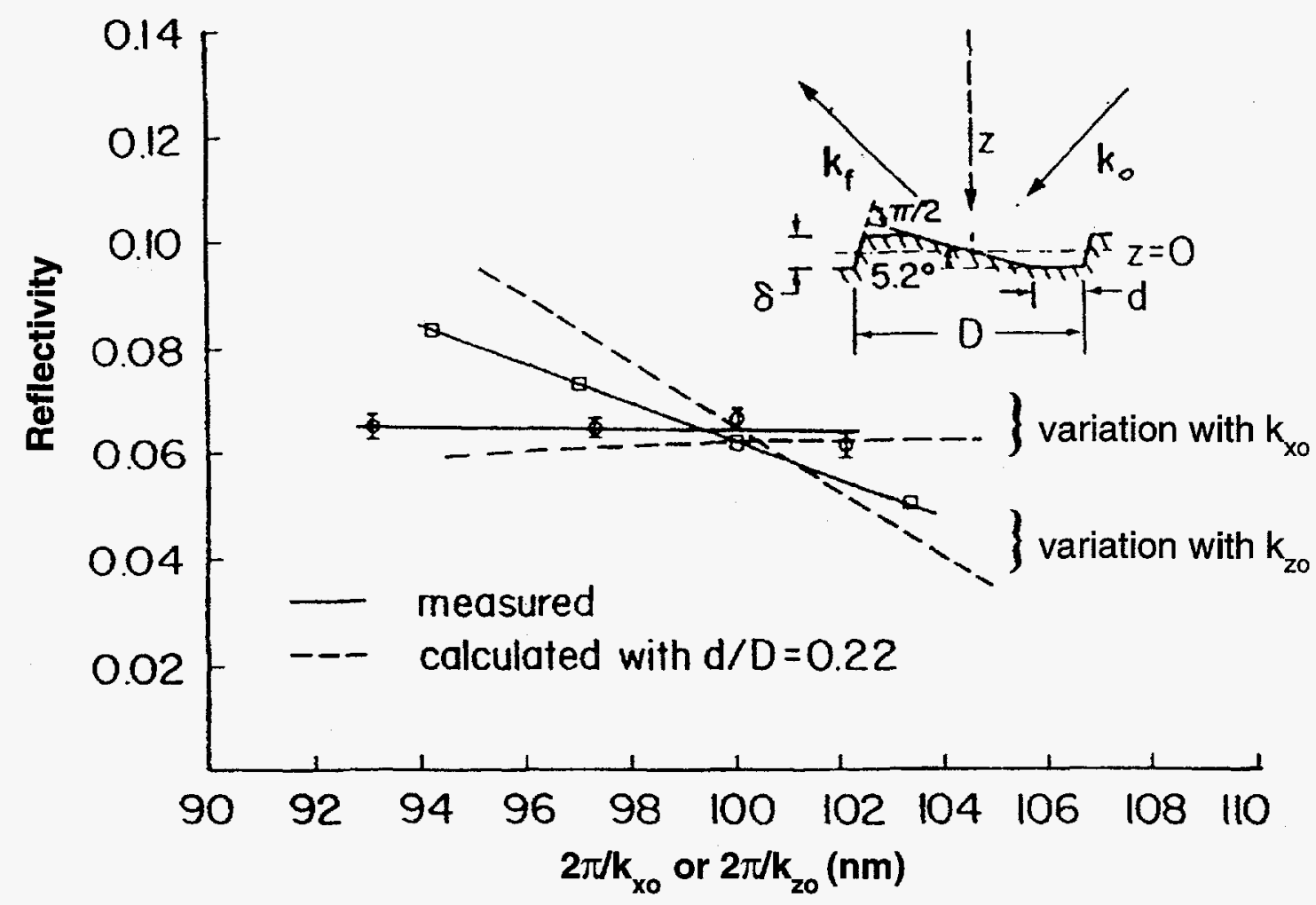

Fig. 15. Measured specular reflectivity of a grating whose groove profile is given on the top right of the figure. The reflectivity was studied with the gravity diffractometer as a function of $k_{z 0}$ and $k_{x 0}$. Experimental data points are represented as open circles (variation with $k_{x 0}$ ) and open squares (variation with $k_{z 0}$ ) and solid lines are drawn through the data points for each case. Calculations using the Rayleigh approximation with $d / D=0.22$ are shown as dashed lines (Steyerl et al. 1992).

First-order diffraction lines were studied with a nickel-coated grating (effective size $10 \times 20 \mathrm{~cm}^{2}$ ) with 1200 grooves $/ \mathrm{mm}$ blazed for a wavelength of $1200 \AA$. The grooves have a slightly asymmetrical rectangular saw-tooth-like profile with a face angle of $5.2^{\circ}$ relative to the surface plane. The results are shown on Fig. 16, where it can be seen that the diffraction lines $\mathrm{n}=-1,0$, and +1 are clearly separated. The intensity of the $0^{\text {th }}$-order line (about $6 \%$ that for total reflection) is in agreement with calculations. The intensities for both the $\mathrm{n}$ $=+1$ and $\mathrm{n}=-1$ lines are, however, a factor of 3 below the expected values. No satisfactory explanation has been provided for this discrepancy. 


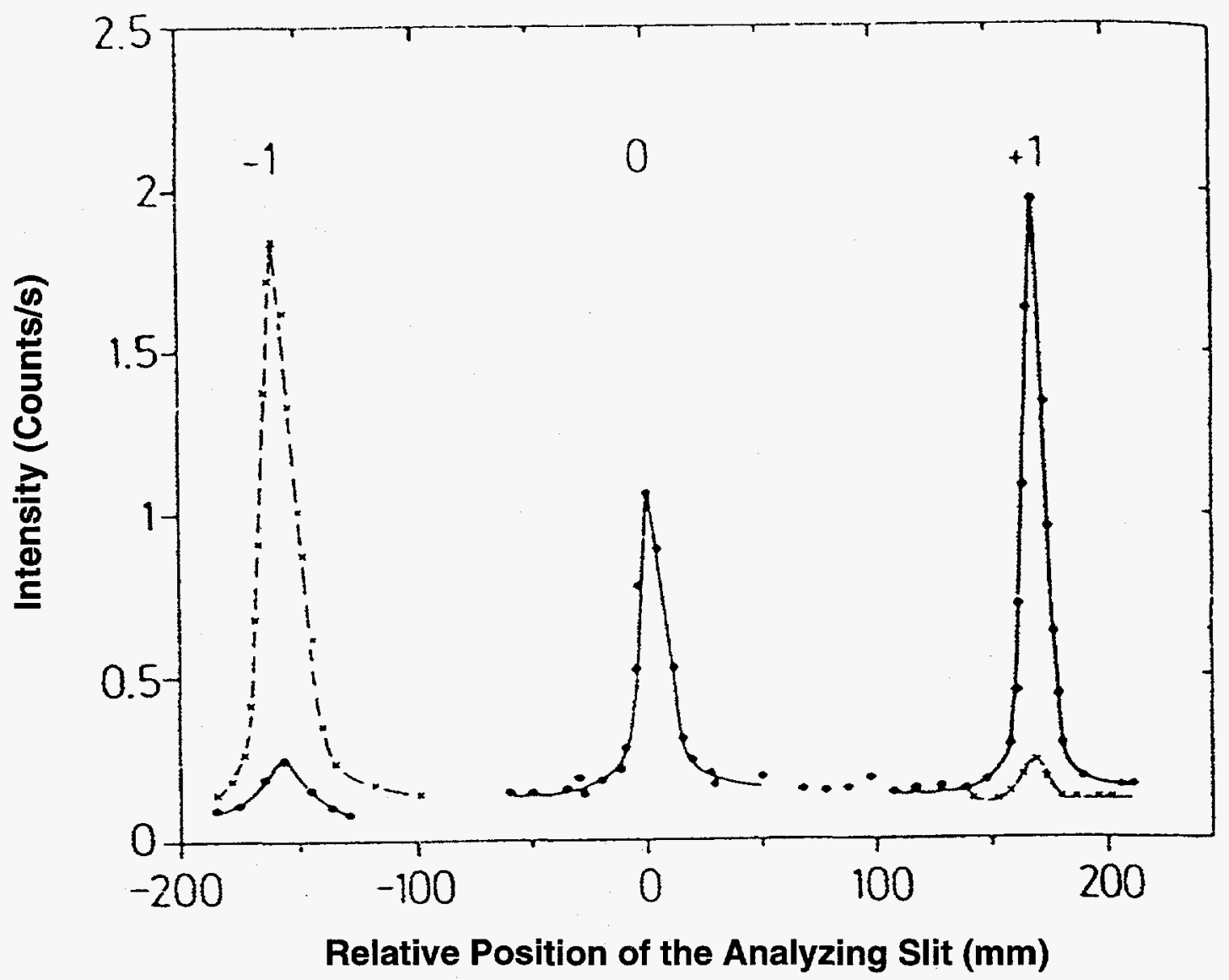

Fig. 16. Diffraction lines of order $n=-1,0$, and +1 for UCNs diffracted by a nickel-coated reflection grating. The measurements were made with the neutron gravity diffractometer installed at the ILL. The FWHM of the diffraction lines is about 1 neV of gravitational energy. The solid and dashed lines are for normal and reversed grating orientation, respectively (Steyerl 1989). This figure is reprinted with kind permission from Elsevier Science - NL, Sara Burgerhartstraat 25, $1055 \mathrm{KV}$ Amsterdam, The Netherlands.

\section{Reflectivity from Thin Films}

Reflectivity from thin films can be studied in the same manner as from thick samples. Whereas reflectivity from thick samples exhibits the simple monotonic behavior shown in Fig. 11, reflectivity from thin films shows a more complicated pattern coming from interference of the neutron waves reflected by the two surfaces of the films. An example of such behavior is given in Fig. 17 for the reflectivity of a thin film of gold evaporated on glass. The interference pattern is sensitive to the film thickness, as predicted by Eq. (25). The data of Fig. 17 can be fitted with Eq. (25) using a film thickness of $d=2680 \pm 60 \AA$. The reflectivity that would be obtained with a thick sample of gold is also plotted in Fig. 17 for comparison. As can be seen from these data, reflectivities below about 0.1 are very difficult to obtain with this instrument when it is installed at the FRM. A better sensitivity to reflectivity would be obtained at the ILL and with future, more intense UCN sources. 


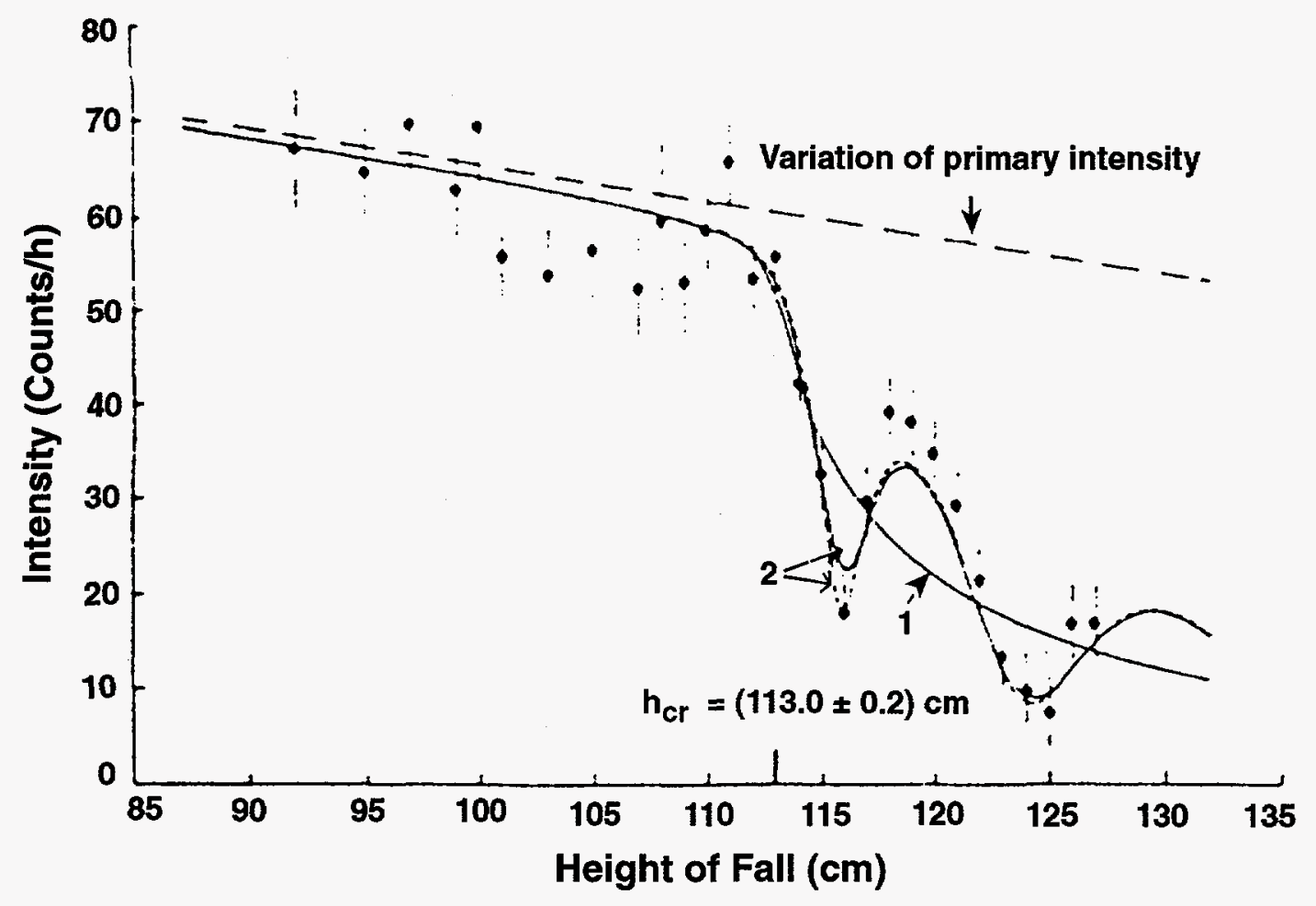

Fig. 17. Measured intensity of UCNs reflected from a glass mirror with a thin gold coating (solid circles with error bars) as a function of the height of fall of the incident neutrons. The measurements were made with the neutron gravity diffractometer installed at the FRM. Solid curve 1 shows calculations of the reflected intensity for a thick gold sample. Curves 2 (dashed and solid) show calculations of the reflected intensity for a thin gold film deposited on a glass mirror. Whereas dashed curve 2 is for monoenergetic neutrons, solid curve 2 is corrected for instrumental resolution (Scheckenhofer and Steyerl 1977).

\section{E. Reflectivity from and Transmission through Multilayer Systems}

Reflectivity from and transmission through composite films have been studied with the UCN gravity spectrometer at the FRM.

Multilayer systems were studied that were composed of a thin film of aluminum sandwiched between two copper films. These three films were evaporated on substrates of glass (reflectivity measurement) and silicon (transmission measurement). Each system was protected by an additional layer of aluminum that was evaporated at the top of the system. Because the UCN potential is larger for copper than for aluminum, the $\mathrm{Cu}-\mathrm{Al}-\mathrm{Cu}$ system behaved as a double-hump potential barrier inside which quasi-bound neutron states could exist (Sec. III.B.7). In these measurements, the resolution was $3 \mathrm{neV}$ (FWHM), and the count rate obtained with a $10 \times 20 \mathrm{~cm}^{2}$ nickel-coated glass mirror was 45 counts $/ \mathrm{h}$, about half the value of the best rates obtained in previous experiments. 


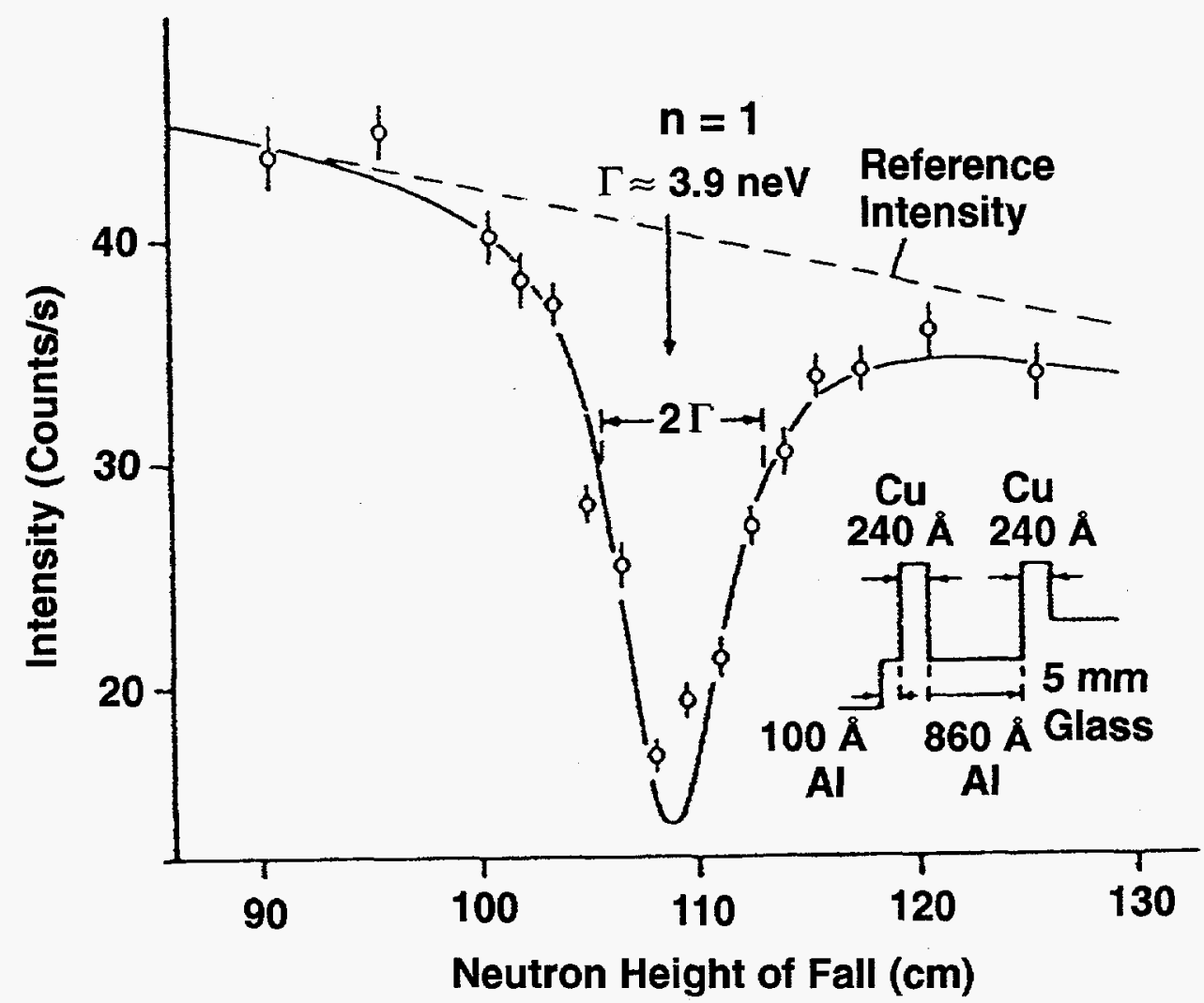

Fig. 18. Measurement of the reflected UCN intensity for a multilayer system as a function of the height of fall of the incident neutrons (open circles with error bars). The measurements were made with the neutron gravity diffractometer installed at the FRM. The solid curve is an exact solution of the one-dimensional Schrödinger equation for the multistep neutron potential of the system shown in the insert.

This curve includes corrections for the effect of instrumental resolution (Steinhauser et al. 1980).

The sample studied in the reflectivity mode was composed of the following layers from top to bottom: $\mathrm{Al}(100 \AA), \mathrm{Cu}(240 \AA), \mathrm{Al}(860 \AA)$, and $\mathrm{Cu}(240 \AA)$ on a glass substrate (see insert in Fig. 18). The reflectivity of this system as a function of neutron vertical energy (or height of neutron fall) is plotted in Fig. 18. The reflectivity curve clearly shows a dip at $108.5 \mathrm{~cm}$, which corresponds to a stationary state in the potential well. This dip is fairly well reproduced by the solid curve, which represents a solution for quantum number $\mathrm{n}=1$ of the one-dimensional Schrödinger equation for this potential. The observed total width of $\approx 7.8 \mathrm{neV}$ corresponds to a lifetime for this quasi-bound state of $\approx 2 \times 10^{-7} \mathrm{~s}$.

The sample studied in the transmission mode is described in Fig. 3 and shown as the insert in Fig. 19. The layer thicknesses (especially the thickness of the central Al layer) make it possible to observe the $n=1$ and the $n=2$ quasi-bound states. The silicon substrate is fairly transparent so as to make transmission measurements possible. The experimental results (Fig. 19) clearly show the peaks corresponding to the $n=1$ and $n=2$ quasi-bound states. The solid curve in Fig. 19 is calculated for a multistep potential. 


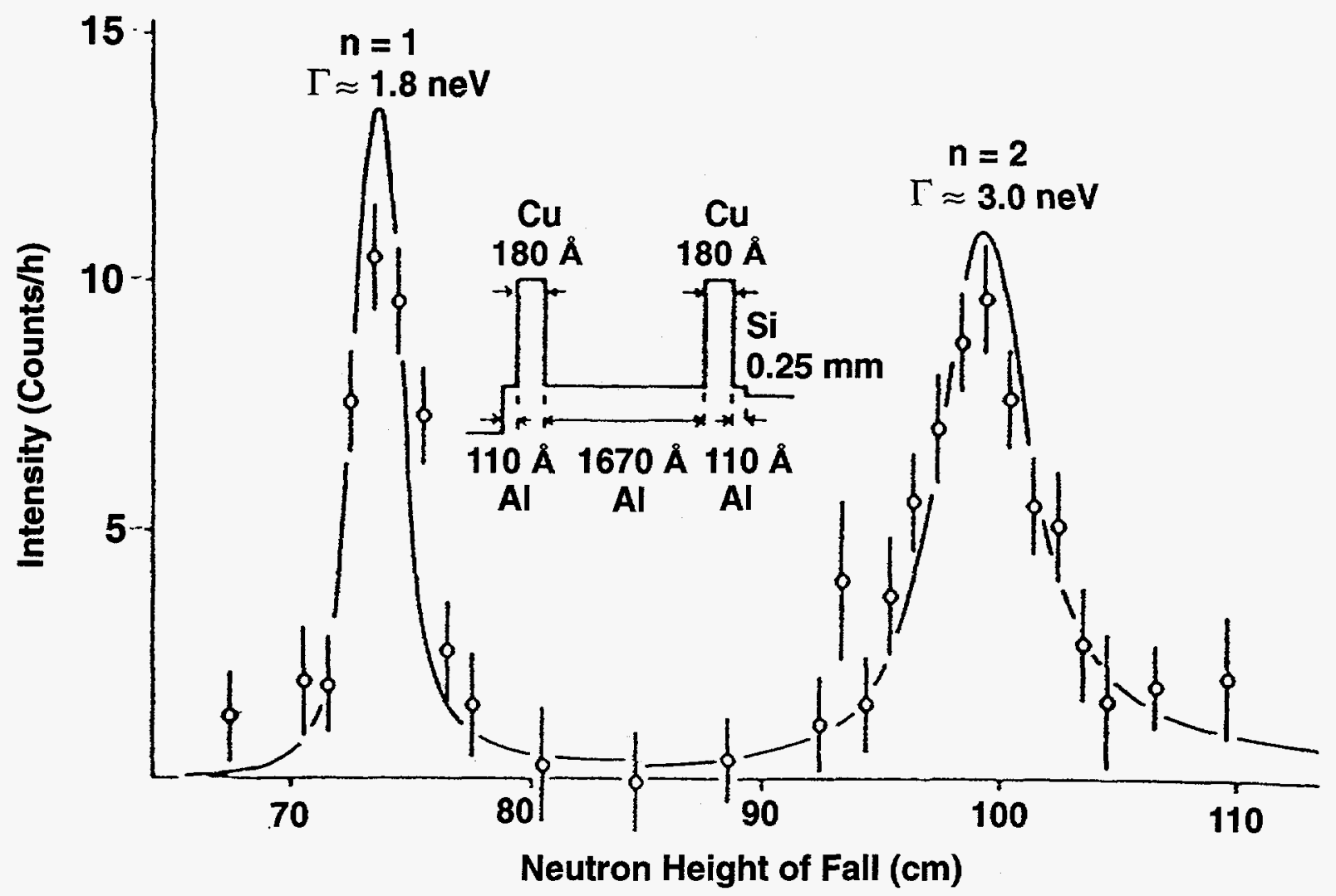

Fig. 19. Measured neutron transmission for a multilayer system as a function of the height of fall of the incident neutrons (open circles with error bars). The measurements were made with the neutron gravity diffractometer installed at the FRM. Two resonances occur at neutron heights of fall of 74 and $99 \mathrm{~cm}$. The resonance widths are equal to $2 \Gamma$ with values of $\Gamma$ given on the figure for each resonance. The solid curve results from calculations using the multistep potential shown in the insert (Steinhauser et al. 1980).

The optical analog of this neutron double-hump-potential device is the classical FabryPerot interferometer. The neutron version of this interferometer could feasibly have a resolution of about $1 \mathrm{neV}$.

More complex systems were studied, such as coupled neutron resonators with multilayers represented by double potential wells. For example, a system studied in the transmission mode with the UCN gravity diffractometer had the following layer sequence from top to bottom: $\mathrm{Al}(100 \AA), \mathrm{Cu}(170 \AA), \mathrm{Al}(600 \AA), \mathrm{Cu}(200 \AA), \mathrm{Al}(600 \AA), \mathrm{Cu}(170$ $\AA$ ), and $\mathrm{Al}(100 \AA$ ) on a silicon substrate (see insert in Fig. 20). The measured transmission of this sample is plotted as a function of neutron energy in Fig. 20. The $n=0$ stationary state, which would appear as a single resonance for a single resonator, now appears split because of the coupling between the two identical resonators through the $200-\AA$ central $\mathrm{Cu}$ layer. The observed split is $\Delta=6.3 \mathrm{neV}$. The exact solution of the one-dimensional Schrödinger equation for this multistep potential is plotted as a dashed line in Fig. 20. The experimental data have the same shape but are significantly below the exact solution because of experimental effects. 


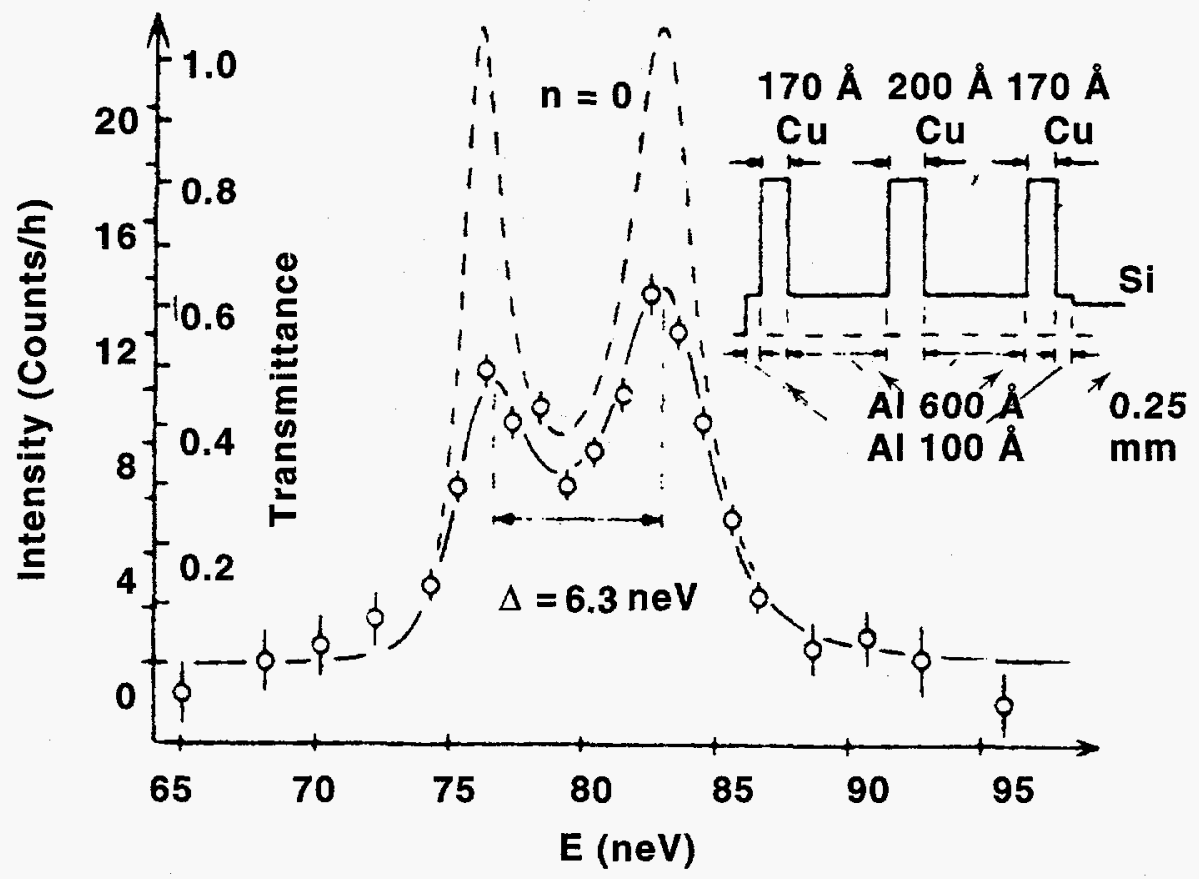

Fig. 20. Measured neutron transmission through a multilayer system whose neutron potential is shown in the insert. The data points (open circles with error bars) are plotted as a function of the vertical energy of the incident neutrons. The measurements were made with the neutron gravity diffractometer installed at the FRM. The solid curve is a phenomenological fit to the data. The dashed curve is an exact solution to the Schrödinger equation used with the potential shown in the insert. The experimental data are lower than the calculations because of instrumental resolution and scattering from rough surfaces (Steyerl et al. 1981).

\section{F. Reflectivity from Magnetic Samples}

Because of the neutron magnetic moment, polarized neutron reflectivity (PNR) can be very useful to measure the magnetic profile near the surface of magnetic materials. Although UCNs could be used for such studies, very few UCN measurements have been reported in this field. Instead, thermal or cold neutron beams were used for PNR at grazing angles.

PNR measurements at grazing angles are illustrated in Fig. 21 for a ferromagnetic sample, which is a single crystal of nickel whose surface that is exposed to the neutron beam is in the (110) plane. Magnetization inside the sample is saturated in the (111) direction in the plane of the surface by a small external magnetic field. Reflectivity is plotted as a function of neutron wavelength for an angle of incidence $\theta_{1} \approx 0.7^{\circ}$ for neutron polarizations parallel and antiparallel to the sample magnetization (Felcher et al. 1987). Such data, possibly supplemented by other measurements made under different physical conditions (temperature, magnetic field), can be used to determine both the magnetic and chemical profiles of a sample.

Another PNR example is given in Fig. 22; the sample is a $300-\AA$ magnetized film of permalloy on top of an antiferromagnetic film of $\mathrm{Ni}_{0.5} \mathrm{Co}_{0.5} \mathrm{O}$. The permalloy sample at a temperature of $20 \mathrm{~K}$ is magnetized parallel to an external magnetic field. PNR measurements were made with the neutron spin parallel and antiparallel to this external field. 


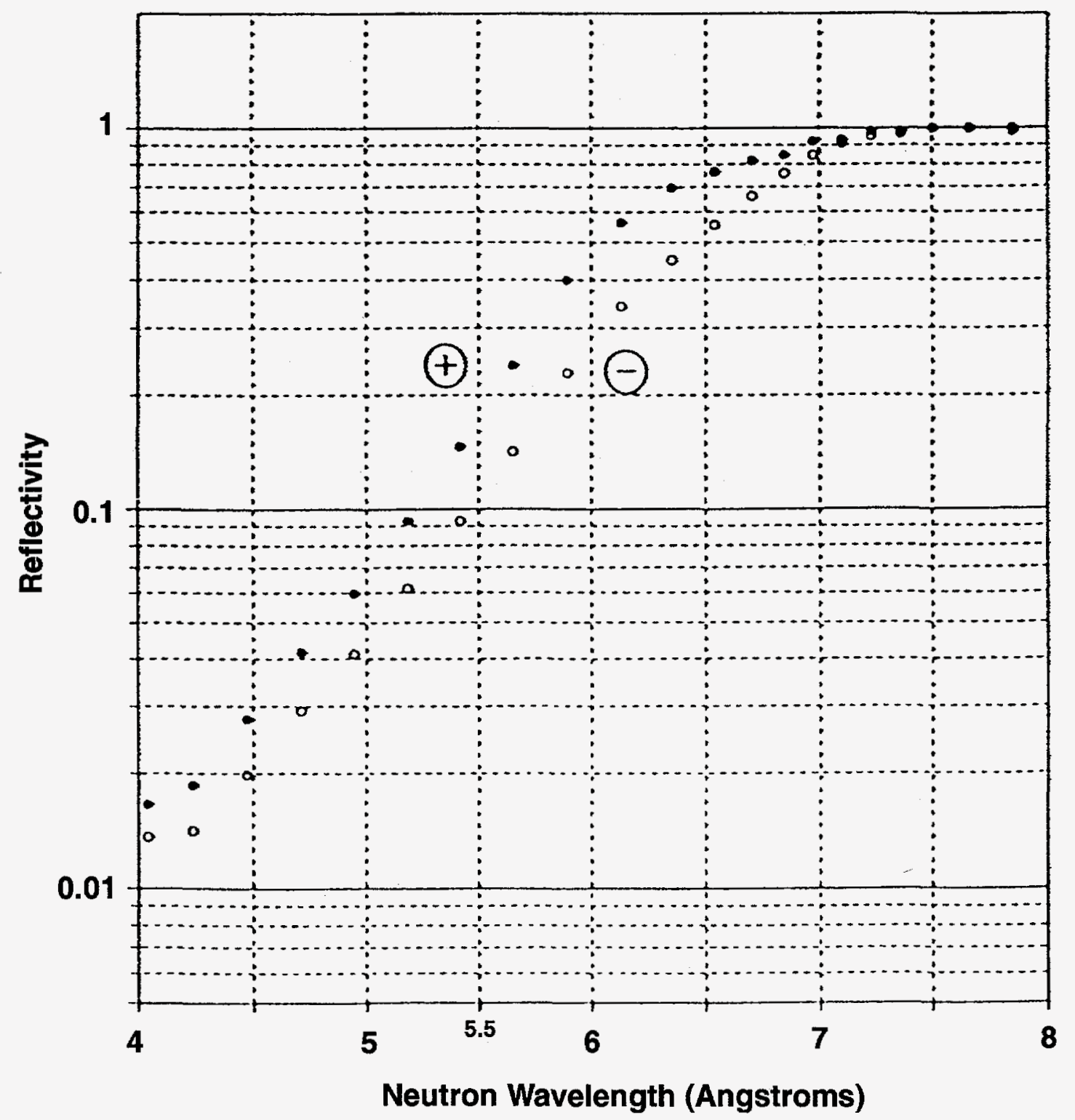

Fig. 21. Measured reflectivity, as a function of neutron wavelength, of polarized neutrons incident on a nickel single crystal. The measurements were made with a grazing-angle neutron reflectometer installed at the IPNS. The spin-dependent reflectivities were measured with the spin of the incident neutrons parallel (solid circles) and antiparallel (open circles) to an external magnetic field of 20 Oe (Felcher et al. 1987). 


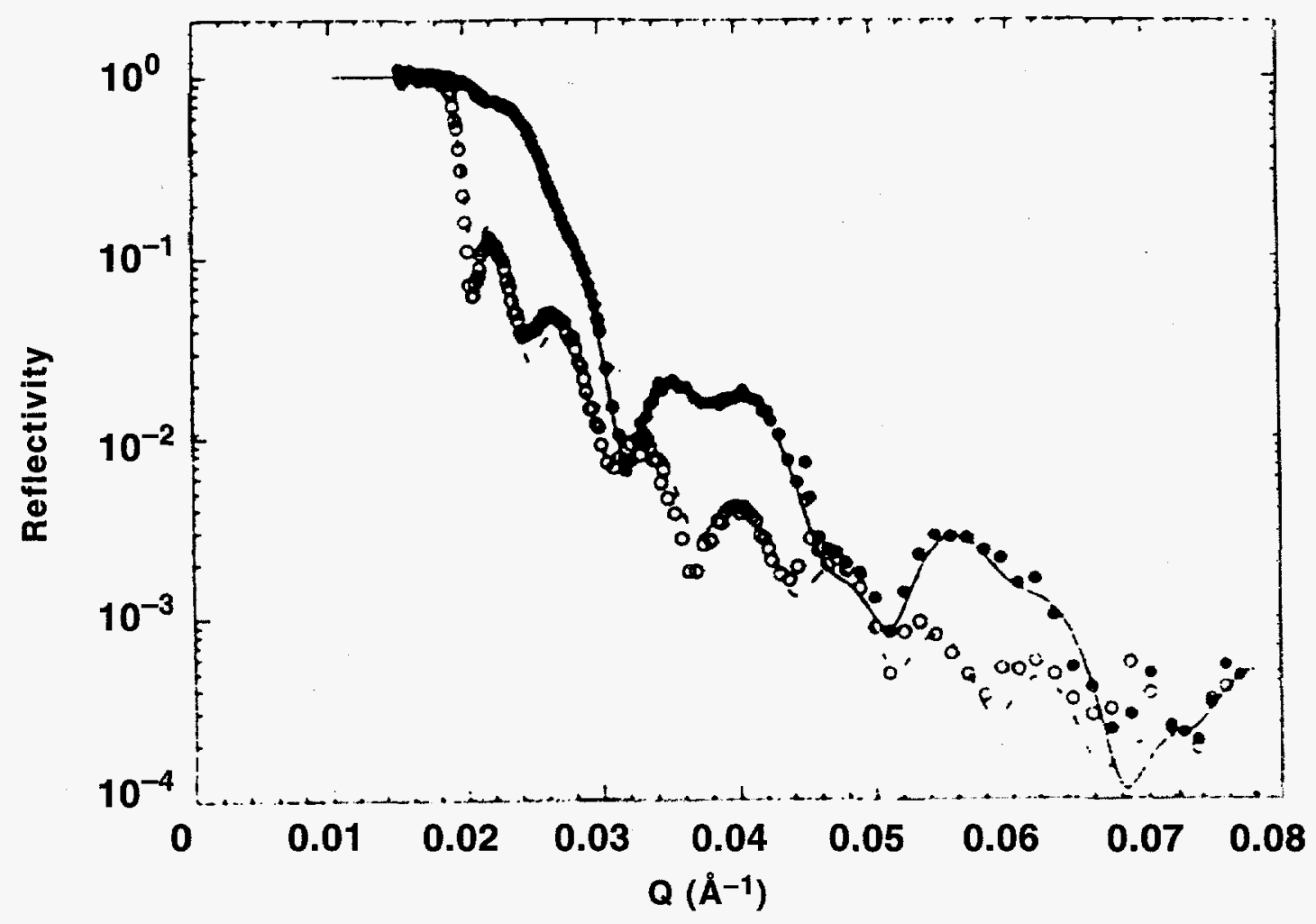

Fig. 22. Measured reflectivity, as a function of momentum transfer, of polarized neutrons incident on a magnetized film of permalloy on top of an antiferromagnetic film of $\mathrm{Ni}_{0.5} \mathrm{Co}_{0.5} \mathrm{O}$. The permalloy is magnetized in the direction of an external magnetic field. The measurements were made with a grazing-angle neutron reflectometer installed at the IPNS. The spin-dependent reflectivities were measured with the spin of the incident neutrons parallel (solid circles) and antiparallel (open circles) to the external magnetic field (Felcher 1993). This figure is reprinted with kind permission from Elsevier Science - NL, Sara Burgerhartstraat 25, 1055 KV Amsterdam, The Netherlands.

A third PNR example was obtained with the surface profile analysis reflectometer (SPEAR) described in Sec. VII. The measurements were made with a $267-\AA$-thick film of iron grown on a polished single crystal of $\mathrm{MgO}$, with the neutron spin either parallel or antiparallel to the magnetic field applied to the sample. The data obtained in these measurements are plotted in Fig. 23. Analysis of these data showed that the iron film had a 25- $\AA$-thick surface oxide of $\mathrm{Fe}_{3} \mathrm{O}_{4}$ or $\gamma-\mathrm{Fe}_{2} \mathrm{O}_{3}$ with an average magnetic moment of $1.6 \mu_{\mathrm{B}}$, a 208 -Å-thick Fe region with an average magnetic moment of $1.8 \mu_{\mathrm{B}}$ (compared with $2.2 \mu_{\mathrm{B}}$ for bulk $\mathrm{Fe}$ ), and a phase boundary region (which was $66 \%$ dense) with an enhanced average magnetic moment of $2.5 \mu_{\mathrm{B}}$. These values of the average magnetic moment are partially related to the changes of the Fe densities in these various layers.

With these types of studies, it is possible to determine the magnetic profiles of bulk samples with a resolution of $50 \mathrm{G}$ and to detect the magnetization of a ferromagnetic monolayer at the level of $0.5 \mu_{\mathrm{B}}$ per atom. Only a few exploratory magnetic measurements have been made with polarized UCNs using the neutron gravity diffractometer at the ILL (Herrmann 1990). 


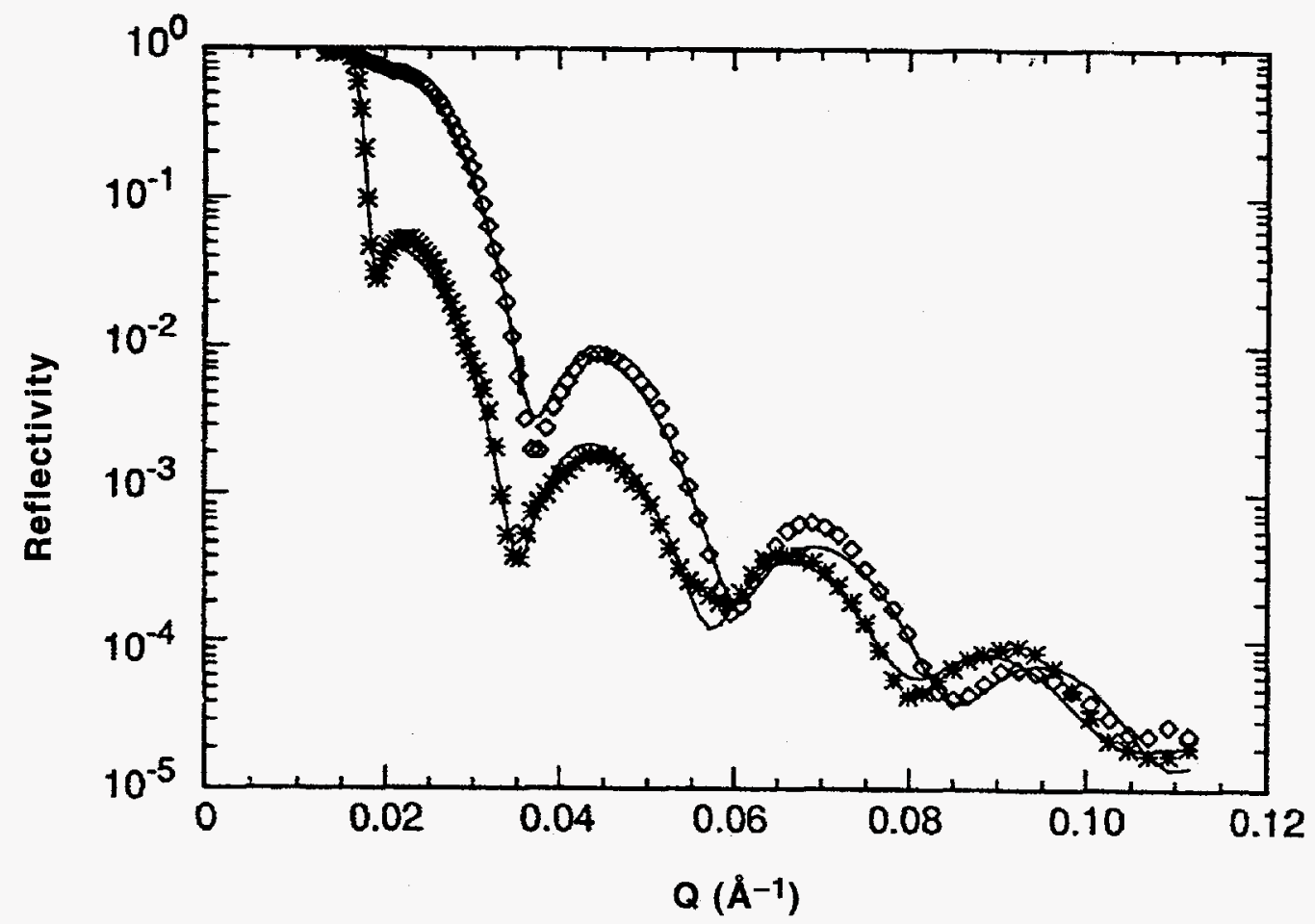

Fig. 23. Measured reflectivity, as a function of momentum transfer, of polarized neutrons incident on an iron film grown on a crystal of magnesium oxide. The measurements were made with SPEAR. The spin-dependent reflectivities were measured with the spin of the incident neutrons parallel (stars) and antiparallel (open diamonds) to the external magnetic field applied to the sample. Analysis of the data gives the magnetic profile of the iron film. Solid lines represent model calculations (From the Tip of the LANSCE 1993).

\section{G. Quasi-Elastic Scattering}

As mentioned in Sec. IV, neutron quasi-elastic scattering is a powerful and promising technique to study the dynamics of macromolecules. The use of UCNs in these studies can supplement other similar studies carried out with high-resolution neutron instruments such as backscattering and NSE spectrometers (Sec. VII). With UCNs it is possible to make measurements at very small momentum transfers ranging from 0.01 to $0.03 \AA^{-1}$ (corresponding to spatial dimensions of a few $100 \AA$ ) and very small energy transfers in the $0.01-n e V$ range. An example of the study of molecular dynamics of lipid bilayers with NESSIE is given next.

The first sample used in this experiment was a bilayer of the lipid dipalmitoyl-phosphatidyl-choline (DPPC) in $\mathrm{D}_{2} \mathrm{O}$ solution with an area of $5 \times 15 \mathrm{~cm}^{2}$ and a thickness of $0.1 \mathrm{~cm}$. In this experiment, NESSIE was used with a resolution of $16.4 \pm 0.5 \mathrm{neV}$. A typical quasielastic peak obtained in these measurements is shown in Fig. 24 for the DPPC sample at a temperature of $41^{\circ} \mathrm{C}$ at which the bilayer is in a liquid crystalline phase. The data can be explained by a broadening of the elastic peak by a Lorentzian having a total width of $\Delta \mathrm{E}=2.1 \pm 1.0 \mathrm{neV}$. Several measurements were made at different temperatures to study 


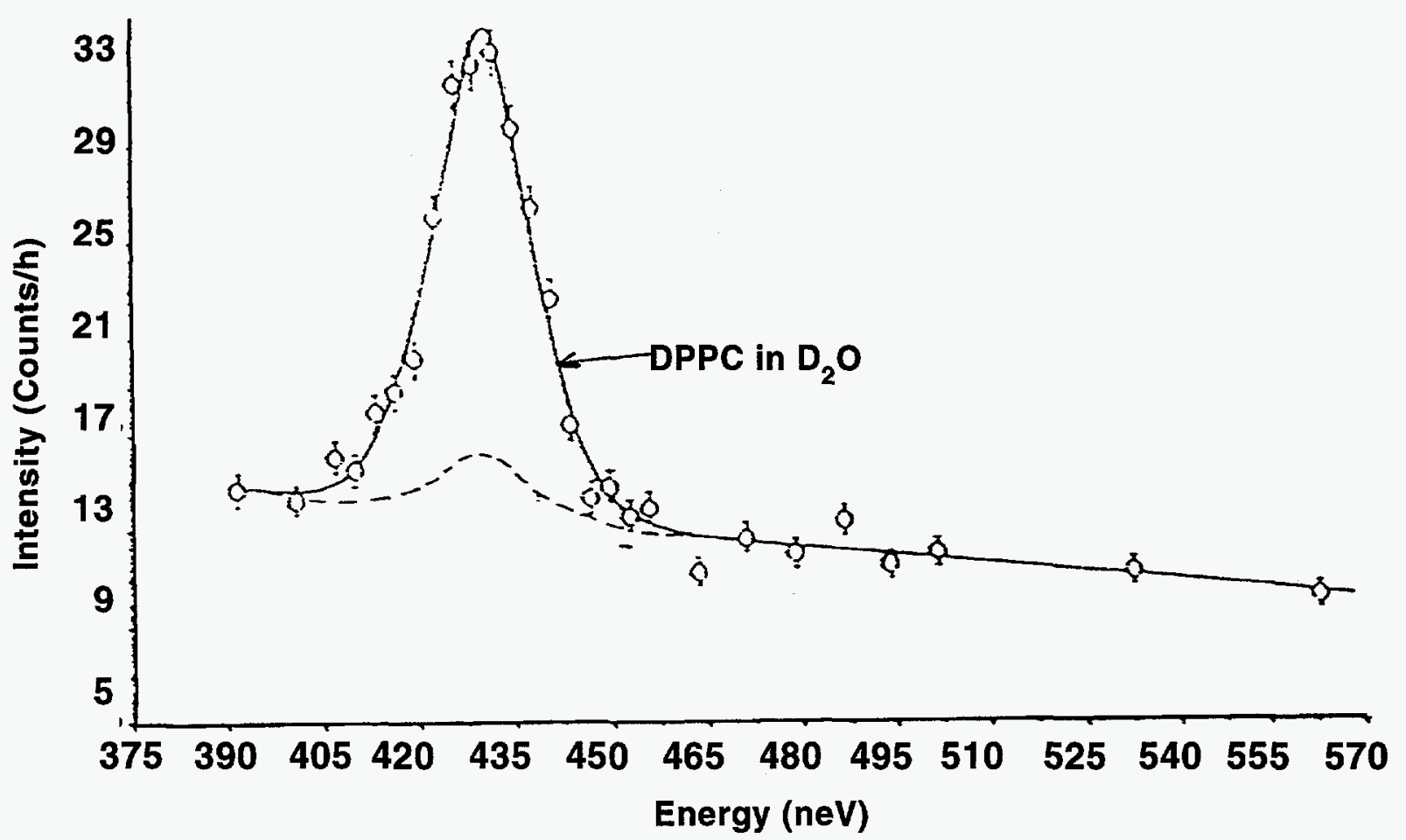

Fig. 24. Spectrum of quasi-elastic scattering of UCNs by a bilayer of DPPC at $41^{\circ} \mathrm{C}$ (open circles with error bars). The dashed line corresponds to the background. The data were obtained with the UCN spectrometer NESSIE installed at the FRM. The solid line is a Lorentzian fit to the experimental quasi-elastic peak (Pfeiffer et al. 1988).

the broadening of the quasi-elastic peak as a function of temperature. No broadening is observed at room temperature, but a significant broadening is observed at temperatures above $40^{\circ} \mathrm{C}$.

Measurements of the broadening of the quasi-elastic peak as a function of momentum transfer were made with bilayer dispersions of a 1:1 mixture of dimyristoyl-phosphatidylcholine (DMPC) and the photopolymerized butadiene phospholipid in pure $\mathrm{D}_{2} \mathrm{O}$, as shown in Fig. 25. Combined with similar data obtained with an NSE spectrometer for relatively high momentum transfers, the UCN data obtained for lower Q values show a linear behavior of the width $\Delta \mathrm{E}$ as a function of $\mathrm{Q}^{2}$. Several dynamical processes were envisaged to explain this broadening and its $\mathrm{Q}^{2}$ dependence. The most likely process seems to be lateral diffusion of the lipid molecules in the plane of the membrane. Such diffusive processes actually produce a Lorentzian broadening with a width that varies linearly with the square of the momentum transfer (see Sec. IV.C.2). By analyzing the results in terms of a twodimensional diffusion model, one can obtain the diffusion coefficient $\mathrm{D}_{\mathrm{T}}$ at various temperatures. At $\mathrm{T}=45^{\circ} \mathrm{C}$, one thus obtains $\mathrm{D}_{\mathrm{T}}=2.6 \times 10^{-7} \mathrm{~cm}^{2} \mathrm{~s}^{-1}$. At the same temperature, a similar value $D_{T}=(2 \pm 1) \times 10^{-7} \mathrm{~cm}^{2} \mathrm{~s}^{-1}$ was obtained with the excimer probe technique, but a much lower value $\mathrm{D}_{\mathrm{T}}=(9 \pm 1) \times 10^{-8} \mathrm{~cm}^{2} \mathrm{~s}^{-1}$ was oḅtained for DPPC multilayers with the photobleaching (FRAP) technique. The difference may come from the fact that the FRAP technique measures the $\mathrm{D}_{\mathrm{T}}$ coefficient over distances of several $\mu \mathrm{m}$, whereas both the UCN and excimer techniques have access to the lipid lateral motion over distances of several lattice spacings (about $100 \AA$ ), which are ten times shorter.

In spite of its much better sensitivity to any broadening of the quasi-elastic peak, the UCN gravity diffractometer does not seem to have been used for quasi-elastic-scattering studies. 


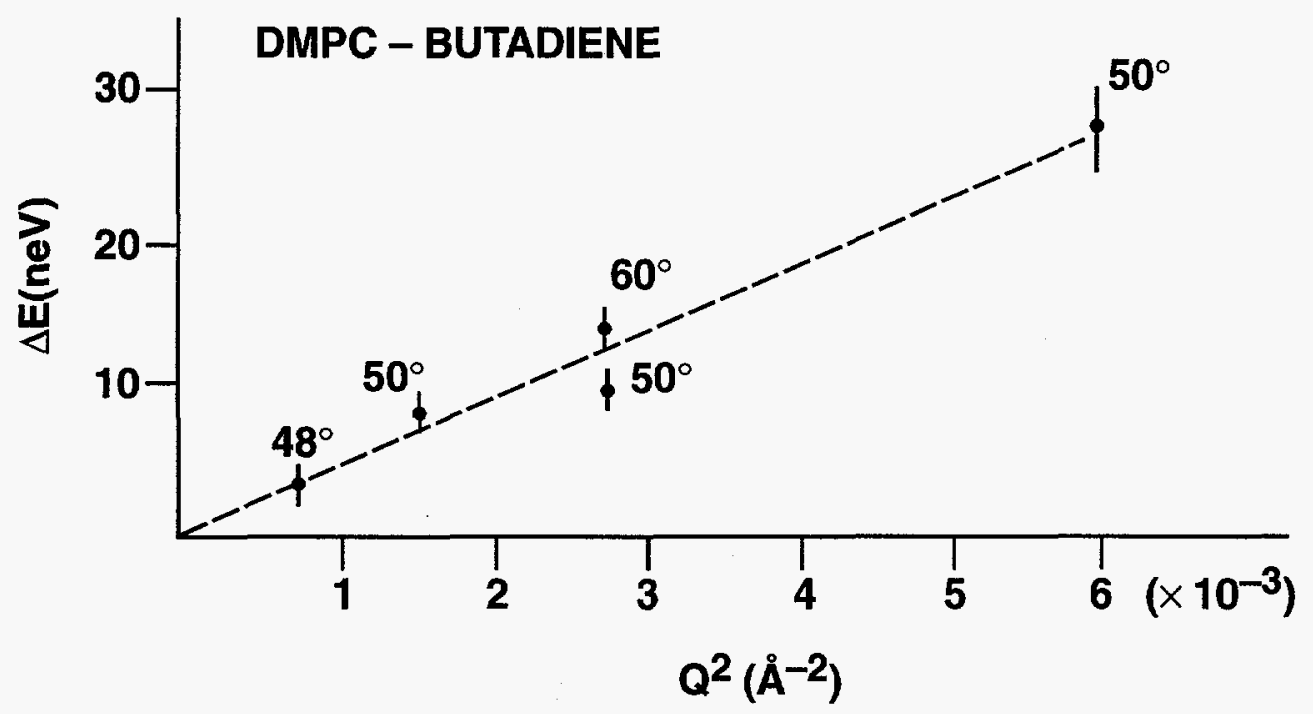

Fig. 25. Measurement of the broadening width $\Delta E$ of the quasi-elastic peak as a function of the square $Q^{2}$ of the momentum transfer for a sample described in the text. The value of $\Delta E$ for the lowest momentum transfer was obtained with NESSIE. The $\triangle E$ values for higher momentum transfers were obtained with the NSE technique. The temperature of the sample is indicated in ${ }^{\circ} \mathrm{C}$ for each measurement (Pfeiffer et al. 1988).

\section{COMPARISON OF ULTRACOLD-NEUTRON INSTRUMENTS WITH OTHER HIGH-RESOLUTION REFLECTOMETERS}

Although a comparison between instruments is very difficult because it depends greatly on the nature and the conditions of the experiments, it is possible to compare some broad features of instruments used to measure similar physical quantities. In this respect, it is interesting to compare the performance of UCN instruments with that of grazing-angle neutron reflectometers, NSE spectrometers, and optical spectrometers. These last three instruments are described in Secs. VII.A, B, and C, respectively, and the performance of these instruments compared with that of UCN spectrometers is summarized in Sec. VII.D. 


\section{A. Grazing-Angle Neutron Reflectometers}

Neutron reflectometers are usually operated with thermal or cold neutrons and, for this reason, must be used at a very small grazing angle to achieve total reflection (see Sec. III.A). As an example, let us consider SPEAR used with cold neutrons and the TOF method at LANSCE. A schematic view of SPEAR is given in Fig. 26. Some results obtained with SPEAR on magnetic materials were illustrated in Fig. 23. The longest-wavelength band (selected by a frame-overlap chopper) that can be used is from 16 to $32 \AA$. At the sample position, the beam cross section is $5 \mathrm{~mm}$ high $\times 25 \mathrm{~mm}$ wide, and the flux for neutrons, whose wavelengths range between 16 and $32 \AA$, is $2 \times 10^{5} \mathrm{n} / \mathrm{cm}^{2}$ s. Reflectivities down to $10^{-6}$ can be measured (Goldstone 1995). Over the whole wavelength range from 1 to $32 \AA$, the momentum transfer varies from 0.007 to $0.3 \AA^{-1}$. The sensitivity to reflectivity and the $\mathrm{Q}$ range of SPEAR are far better than those of UCN instruments. For long wavelengths, however, the count rate with SPEAR decreases very rapidly with increasing wavelength.

A comparison of the count rate versus resolution width for several wavelengths (or energies) is made in Fig. 27 for SPEAR and the UCN gravity diffractometer described in Sec. V.B. Data for SPEAR were provided by Smith (1996). Although SPEAR has a much higher count rate than the UCN diffractometer for resolutions above 5 to $10 \mathrm{neV}$, the UCN diffractometer can attain resolutions in the neV range, down to about $0.5 \mathrm{neV}$, for a wide range of incident vertical energies. Such high resolutions are beyond the reach of SPEAR and similar grazing-angle neutron reflectometers.

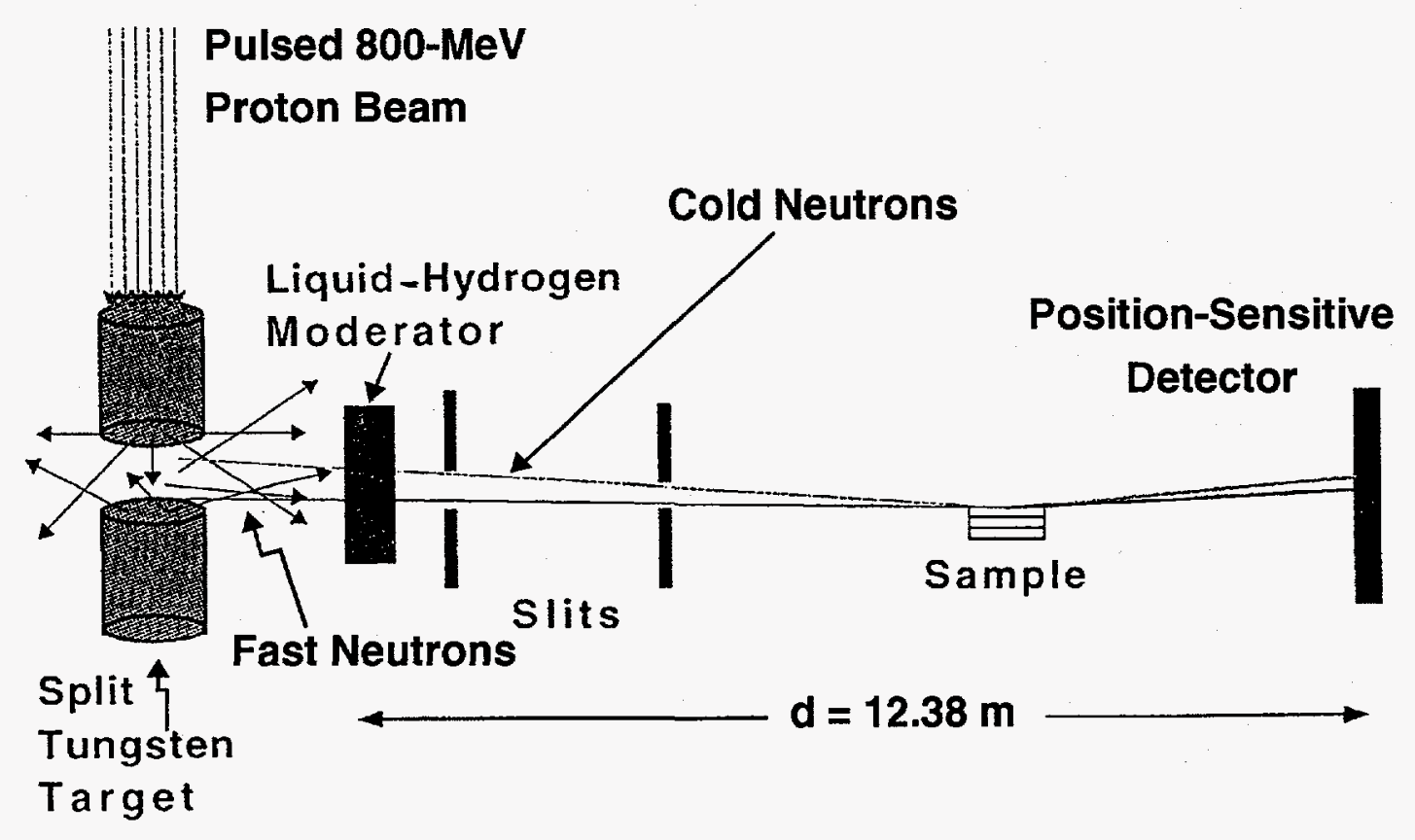

Fig. 26. Schematic of the SPEAR grazing-angle neutron spectrometer (From the Tip of the LANSCE 1993) installed at the LANSCE short-pulse spallation source (SPSS). A collimated beam of cold neutrons from a liquid-hydrogen moderator is incident at grazing angle on a horizontal sample. The angle of the neutrons scattered by the sample is analyzed with a position-sensitive detector; the energy of the neutrons is analyzed by the TOF method. 


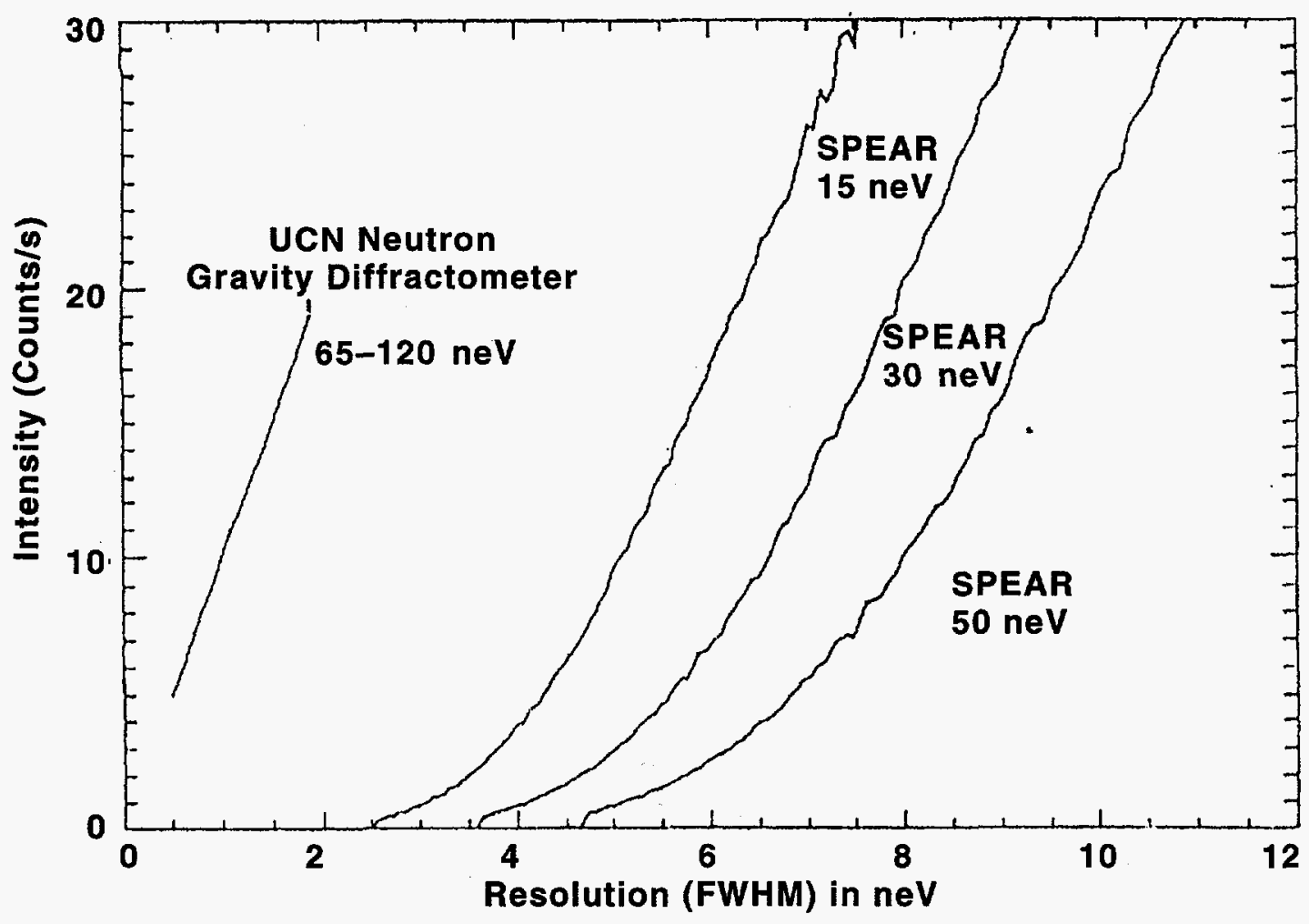

Fig. 27. Count rate versus resolution width (FWHM) for the UCN gravity diffractometer installed at the ILL and SPEAR (Smith 1996). Resolution widths and energies are given for the vertical motion of the incident neutrons. The sample size is $5 \times 15 \mathrm{~cm}^{2}$ for the UCN gravity diffractometer and $25 \mathrm{~cm}^{2}$ for SPEAR.

Because SPEAR is used with the TOF method and with a broad energy band of incident neutrons, no quasi-elastic measurements are possible with this instrument (or any other similar TOF instrument).

A high-resolution grazing-angle neutron reflectometer is being considered at the ILL, which would use a vertical sample and would have a much improved resolution compared with that of SPEAR. This new instrument is still in the design stage, and its exact performance is not yet known (Timmins and Lauter 1996).

\section{B. NSE Spectrometers}

The NSE technique is widely used in high-resolution quasi-elastic scattering measurements (Mezei 1980, Bée 1988). In the NSE technique, the neutron velocities are directly compared before and after scattering, as illustrated in Fig. 28. The incident neutrons are first monochromatized with a fairly broad resolution and then polarized before they enter a region having a longitudinal magnetic field $\mathbf{H}_{0}$. These incident neutrons, whose polarization is perpendicular to $\mathbf{H}_{0}$, undergo Larmor precessions along this magnetic field before reaching the sample. After scattering, the neutrons precess in the opposite sense along another magnetic field $\mathbf{H}_{1}$. The difference between the two precession angles for each neutron results in a change in polarization, which is analyzed at the detector. 


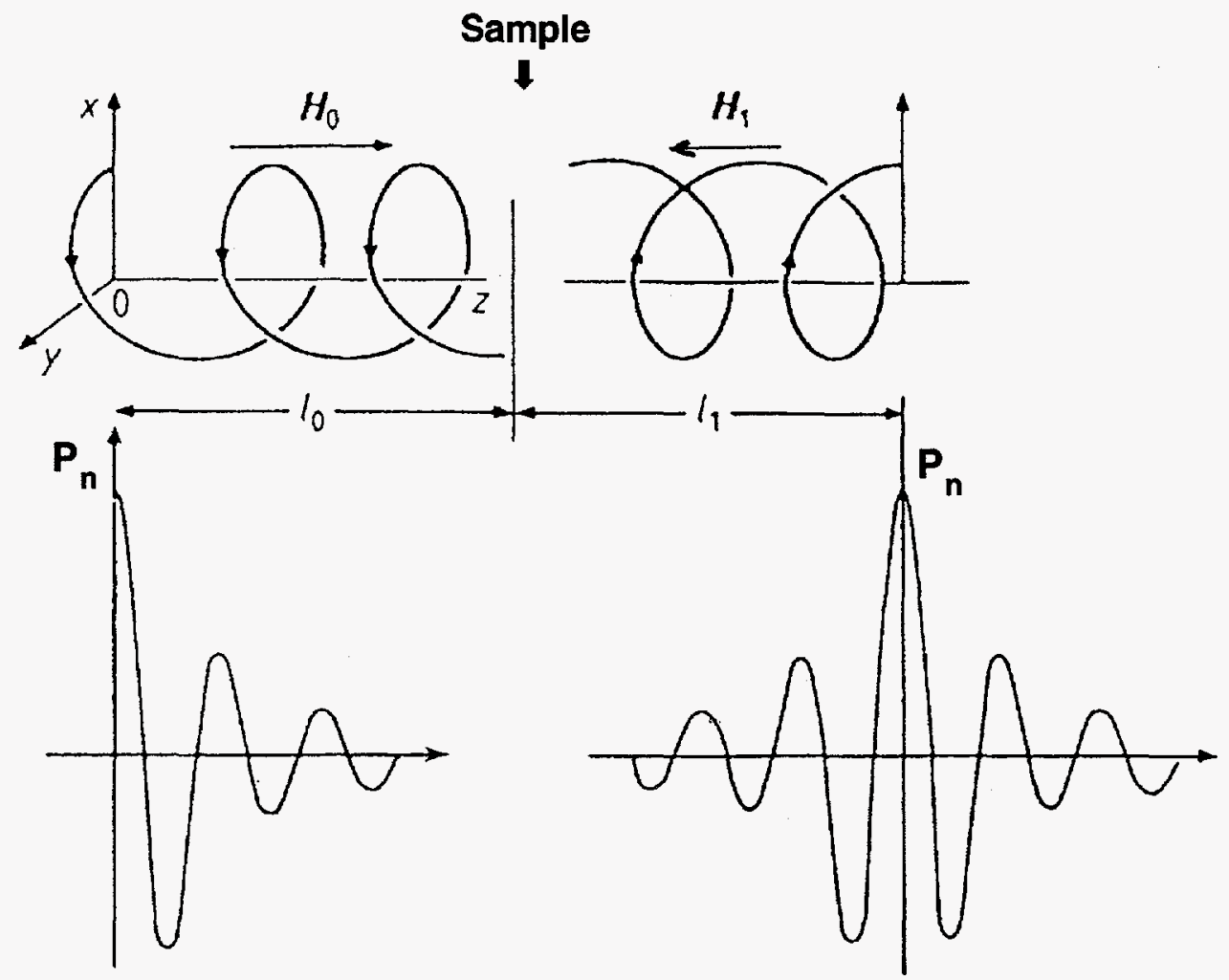

Fig. 28. Larmor spin precession of polarized neutrons along a longitudinal magnetic field before and after scattering by a sample. The neutron polarization $P_{n}$ along the initial polarization axis, which is perpendicular to the magnetic field, is plotted as a function of the distance traveled by the neutrons in the lower part of the figure (Bée 1988).

The precession angle $\varphi$ for a neutron of velocity $\mathrm{v}$ along a distance 1 around a magnetic field $\mathbf{H}_{0}$ is given by the following formula:

$$
\varphi=\gamma_{\mathrm{n}} \frac{1 \mathrm{H}_{0}}{\mathrm{v}},
$$

where $\gamma_{n}$ is the neutron gyromagnetic ratio. The total phase $\phi$ acquired by an incident neutron of velocity $\mathbf{v}_{0}$ traveling a distance $\mathrm{l}_{0}$ in magnetic field $\mathbf{H}_{0}$ and then scattered at velocity $\mathbf{v}_{1}$ before traveling a distance $l_{1}$ in magnetic field $\mathbf{H}_{1}$ (whose direction is opposite to that of $\mathrm{H}_{0}$ ) is the difference between the phase $\varphi_{0}$ acquired along the path of length $1_{0}$ and the phase $\varphi_{1}$ acquired along the path of length $l_{1}$, as given by the following formula:

$$
\phi=\varphi_{0}-\varphi_{1}=\gamma_{\mathrm{n}}\left[\frac{1_{0} \mathrm{H}_{0}}{v_{0}}-\frac{1_{1} \mathrm{H}_{1}}{v_{1}}\right]=\phi\left(\mathrm{v}_{0}, \mathrm{v}_{1}\right) .
$$

The energy transfer $\hbar \omega$ during the scattering process is equal to

$$
\hbar \omega=\mathrm{E}_{1}-\mathrm{E}_{0}=\frac{1}{2} \mathrm{~m}\left(\mathrm{v}_{1}^{2}-\mathrm{v}_{0}^{2}\right)=\hbar \omega\left(\mathrm{v}_{0}, \mathrm{v}_{1}\right)
$$


The phase difference $\phi$ and the energy transfer $\hbar \omega$ are therefore related to each other through Eqs. (67) and (68). The neutron polarization $P_{n}$ at the end of the whole process is

$$
P_{n}=\cos \phi \text {. }
$$

The NSE spectrometer can be set to $\phi=0$ for elastic scattering $\left(v_{0}=v_{1}\right)$. This implies that $1_{0} \mathrm{H}_{0}=1_{1} \mathrm{H}_{1}$. In the case of a small energy exchange $\hbar \omega$ in a process like quasi-elastic scattering, a phase change $\Delta \phi$ will occur as a result of a change in velocity after scattering. By differentiating Eqs. (67) and (68) with respect to $v_{1}$, assuming that $\mathrm{v}_{0}$ is fixed, one obtains the following relation between $\Delta \phi$ and $\omega$ :

$$
\Delta \phi=\frac{\mathrm{h} \gamma_{\mathrm{n}} \mathrm{l}_{0} \mathrm{H}_{0}}{2 \pi \mathrm{mv}_{0}^{3}} \omega=\tau_{\mathrm{NSE}} \omega
$$

with

$$
\tau_{\mathrm{NSE}}=\frac{\mathrm{h} \gamma_{\mathrm{n}} \mathrm{l}_{0} \mathrm{H}_{0}}{2 \pi \mathrm{mv} v_{0}^{3}}
$$

The polarization of the neutron beam at the end of the spectrometer is

$$
P_{n}=\cos \Delta \phi .
$$

For a sample having a scattering function $S(\vec{Q}, \omega)$, which gives the probability that neutrons are scattered with an energy change $\hbar \omega$, for a given momentum transfer $\vec{Q}$, the neutron polarization at the end of the spectrometer is

$$
P_{n}=P_{N S E}=\frac{\int S(\vec{Q}, \omega) \cos \left(\tau_{N S E} \omega\right) d \omega}{\int S(\vec{Q}, \omega) d \omega}
$$

Therefore, the measurement of the final neutron polarization obtained with the NSE technique gives the Fourier transform $\mathrm{P}_{\mathrm{NSE}}$ of the scattering function, i.e., the intermediate scattering function $F(\vec{Q}, t)$ defined in Sec. IV.B. This intermediate scattering function is obtained from several measurements made with different values of the parameter $\tau_{\mathrm{NSE}}$. In the case of pure elastic scattering and no broadening by experimental effects, $P_{n}$ is constant for all values of $\tau_{\mathrm{NSE}}$. In the case of pure elastic scattering measured with a nonzero experimental resolution $R(\Delta \omega), P_{n}$ will show a variation with $\tau_{N S E}$. If $R(\Delta \omega)$ is a Lorentzian

$$
\mathrm{R}(\Delta \omega)=\frac{\mathrm{c}}{\left(\frac{1}{2} \gamma_{\exp }\right)^{2}+(\Delta \omega)^{2}}
$$


of total width $\gamma_{\text {exp }}$, the Fourier transform of this function is an exponential called $\mathrm{P}_{\mathrm{NSE}}^{0}$, which is equal to

$$
\mathrm{P}_{\mathrm{NSE}}^{0}=\exp \left(-\gamma_{\exp } \mathrm{t}\right)
$$

Therefore, the slope of $\log \mathrm{P}_{\mathrm{NSE}}^{0}$ as a function of $t=\tau_{\mathrm{NSE}}$ gives the width of the resolution function. If the scattering function for quasi-elastic scattering is also a Lorentzian of total width $\Gamma$ given by

$$
S(Q, \omega)=\frac{c^{\prime}}{\left(\frac{1}{2} \Gamma\right)^{2}+(\Delta \omega)^{2}}
$$

the Fourier transform of this function is also an exponential $\exp (-\Gamma \mathrm{t})$, which would be directly obtained from $\mathrm{P}_{\mathrm{NSE}}$ measurements made with perfect resolution.

$$
\mathrm{P}_{\mathrm{NSE}}=\exp (-\Gamma \mathrm{t})
$$

The experimental quantity $\mathrm{P}_{\mathrm{NSE}}^{\mathrm{exp}}$ obtained from a measurement of the scattering function given by Eq. (76) with an NSE spectrometer having a resolution function given by Eq. (74) is the product of the exponentials $\exp \left(-\gamma_{\exp } t\right)$ and $\exp (-\Gamma t)$ :

$$
P_{N S E}^{\exp }=P_{N S E}^{0} \times P_{N S E}=\exp \left(-\gamma_{\exp } t\right) \times \exp (-\Gamma t)=\exp \left[-\left(\gamma_{\exp }+\Gamma\right) t\right]
$$

Spectroscopists using the NSE technique often use the concept of spectral resolution, which is defined as the width $\gamma_{\mathrm{sr}}$ of a fictitious narrow quasi-elastic peak which would cause a small broadening of the experimental resolution $R(\Delta \omega)$. Somewhat arbitrarily, this spectral resolution is defined as causing a 5\% decrease of $\mathrm{P}_{\mathrm{NSE}}^{0}$ in the NSE results at the maximum value $\tau_{\max }$ of the time parameter $\tau_{\mathrm{NSE}}$, as illustrated in Fig. 29. Under these conditions,

$$
\begin{aligned}
\mathrm{P}_{\mathrm{NSE}}^{\exp } / \mathrm{P}_{\mathrm{NSE}}^{0} & \left.=\exp \left[-\gamma_{\exp }+\gamma_{\mathrm{sr}}\right) \tau_{\max }\right] / \exp \left(-\gamma_{\exp } \tau_{\max }\right) \\
& =\exp \left(-\gamma_{\mathrm{sr}} \tau_{\max }\right)=0.95
\end{aligned}
$$

This means that the spectral resolution is then defined as

$$
\gamma_{\mathrm{sr}} \times \tau_{\max }=0.05
$$

independent from the width $\gamma_{\exp }$ of the resolution function (Gähler 1995). 


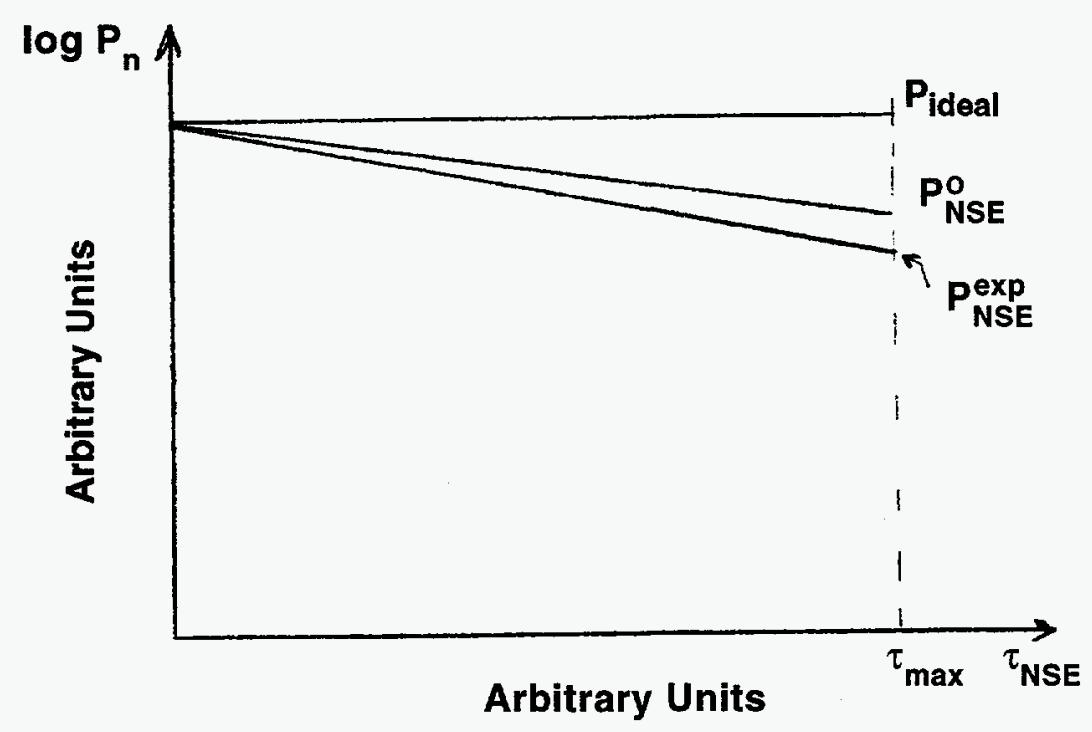

Fig. 29. Usual definition of the spectral resolution of an NSE spectrometer. For pure elastic scattering measured with perfect resolution, the neutron polarization $P_{n}$ versus $\tau_{N S E}$ is constant (called $P_{\text {ideal }}$ on this figure) up to the maximum experimental value $\tau_{\max }$ of $\tau_{\text {NSE. }}$ For pure elastic scattering measured with nonzero experimental resolution, the measured neutron polarization $P_{N S E}^{o}$ decreases as a function of $\tau_{N S E .}$. For quasi-elastic scattering measured with the same experimental resolution, the measured polarization $P_{N S E}^{\text {exp }}$ decreases faster than $P_{N S E}^{O}$ as a function of $\tau_{N S E}$.

Most of the results on quasi-elastic scattering using the NSE technique were obtained with the IN11 NSE spectrometer at the ILL. A schematic of this instrument is given in Fig. 30. The incident neutrons coming from a cold source have wavelengths between $4.5 \AA$ and $12 \AA$. These neutrons are monochromatized (with a wavelength resolution of about $20 \%$ ) with a velocity selector and longitudinally polarized with Fe-Ag supermirrors. A first $\pi / 2$-flipper coil turns the neutron polarization perpendicular to the magnetic field $\mathbf{H}_{0}$. After precessing around the field $\mathbf{H}_{0}$ and scattering from the sample, the neutrons are reversed in polarization by a $\pi$ flipper before they precess in the opposite direction around the field $\mathbf{H}_{1}$. At the end of this precession, the neutron polarization is again turned by a $\pi / 2$ flipper before being analyzed by Co-Ti supermirrors.

The IN1 1 instrument allows a spectral resolution ranging from $3.3 \times 10^{-7} \mathrm{eV}$ at $\lambda=4.5 \AA$ to $1.5 \times 10^{-8} \mathrm{eV}$ at $\lambda=12 \AA$. The momentum transfer ranges from $0.03 \AA^{-1}$ to $2.7 \AA^{-1}$ at $4 \AA$ and from 0.015 to $1.35 \AA^{-1}$ at $8 \AA$. In favorable cases, a quasi-elastic peak broadening down to a few neV has been claimed. The resolution is usually quoted for a small neutronbeam divergence $\left(0.5^{\circ} \times 0.5^{\circ}\right)$, but the resolution deteriorates as the divergence is increased. At the maximum divergence $\left(1.2^{\circ} \times 1.2^{\circ}\right)$, the sample size is $30 \mathrm{~mm}$ in diameter, and the resolution is about $30 \%$ broader than with a small collimation. The count rate is maximum for wavelengths between $4 \AA$ and $5 \AA$ and is then of the order of a few counts per second for a 5\% isotropic scatterer sample (Dagleish et al. 1980). At $9 \AA$, the count rate is reduced by a factor of about 8 . An example of the numerous results obtained with IN11 is given in Fig. 31 where the width of the quasi-elastic peak was measured as a function of momentum transfer for a sample of immunoglobulin in $\mathrm{D}_{2} \mathrm{O}$ solvent. The width is found to vary linearly with $\mathrm{Q}^{2}$, which is typical of a diffusive process for which the diffusion constant $\mathrm{D}_{\mathrm{T}}$ can be obtained (Sec. IV.C). 


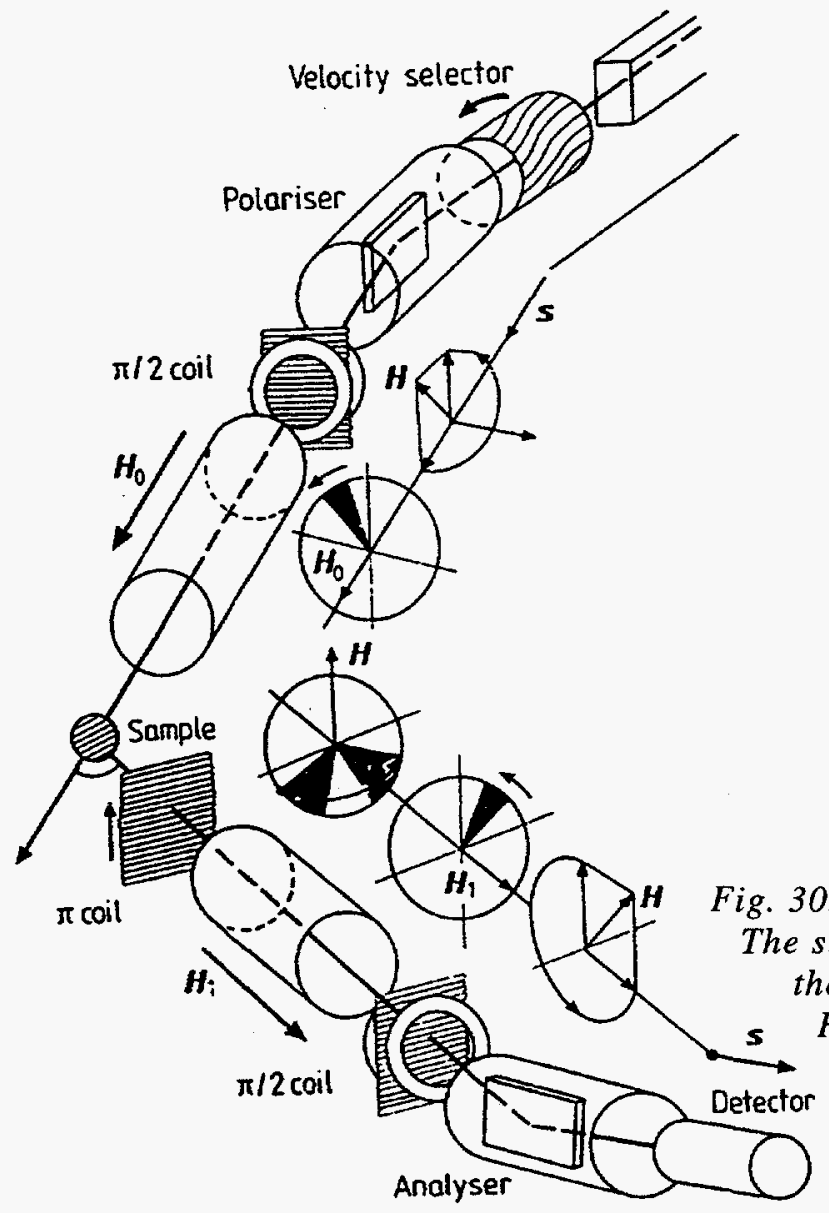

Schematic view of the INII NSE spectrometer. successive transitions of the neutron spin along the instrument are indicated. In contrast to Fig. 28, a $\pi$ neutron flipper is inserted after the sample, and the magnetic field $\mathrm{H}_{1}$ is parallel to $\boldsymbol{H}_{0}$. These modifications do not change the principle of operation of the spectrometer (Bée 1988).

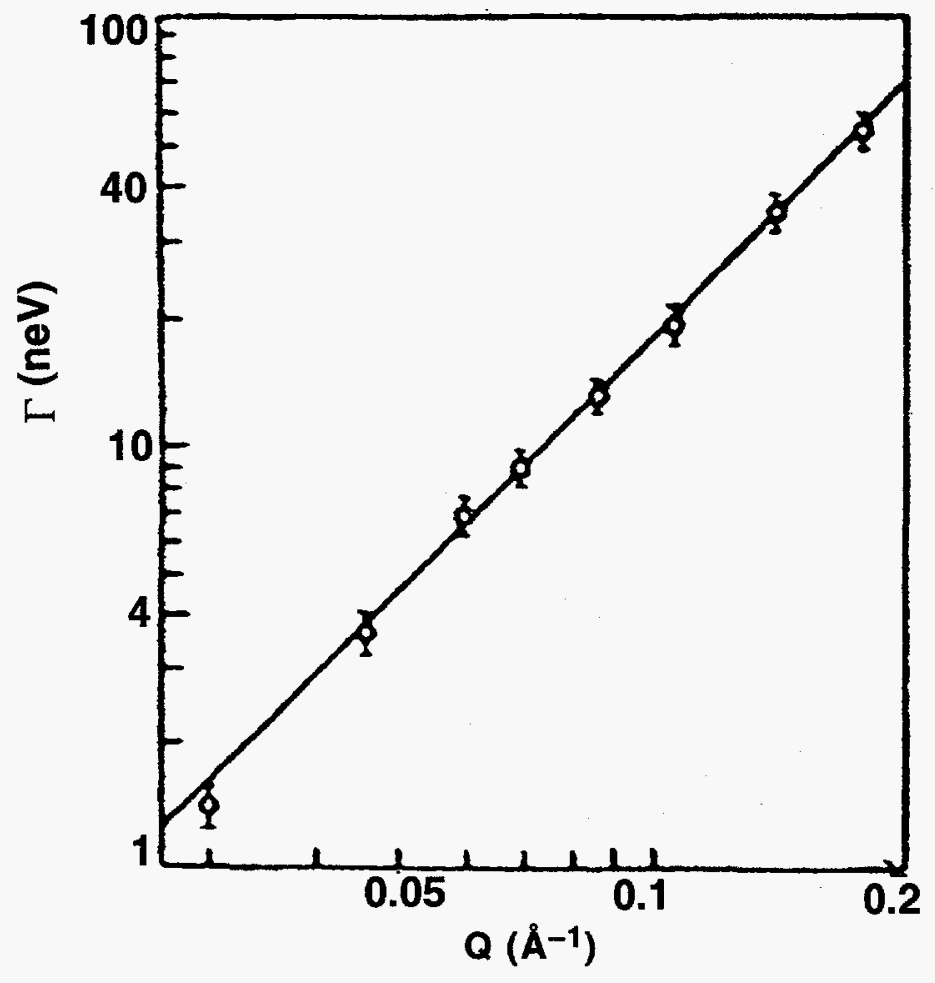

Fig. 31. Total width $\Gamma$ of the quasielastic peak plotted as a function of momentum transfer $Q$, for a sample of immunoglobulin $\mathrm{G}$ in $\mathrm{D}_{2} \mathrm{O}$ solvent. The measurement points (open circles with error bars) were obtained with the IN11 NSE spectrometer. The solid line is a fit to these data (Ibel 1994). 
Another NSE spectrometer was recently constructed at the Laboratoire Léon Brillouin in France with spatial and spectral resolutions improved by about a factor of 2 compared with those of IN11. But a major improvement to the NSE technique will come with a new NSE spectrometer, called IN15, in construction at the ILL to optimize the IN11 design for neutron wavelengths between $8 \AA$ and $20 \AA$. With IN15, which is expected to be operational around 1998, measurements with momentum transfers between $0.001 \AA^{-1}$ and $0.4 \AA^{-1}$ will be possible. Spectral resolutions down to $0.1 \mathrm{neV}$, which is about 20 times better compared with the resolution of IN11, are anticipated. At $8 \AA$, the count rate of IN15 is anticipated to be about twice that of IN11.

The NSE technique has so far proved to be very powerful and is expected to provide resolutions improved by about one order of magnitude in the next few years.

\section{Optical Spectroscopy}

Quasi-elastic light scattering makes possible the observation of translational and rotational motions over scale lengths of the order of the wavelength of light or near-ultraviolet radiation with very small momentum transfers (below $5 \times 10^{-3} \AA^{-1}$ ). Therefore, there is a gap of one order of magnitude between the momentum transfer by optical spectroscopy and that of the low-Q limit of the best existing neutron spectrometers (IN11 and the UCN gravity diffractometer). The lowest momentum transfer attained by the new generation of NSE spectrometers (IN15) will, however, approach that of optical spectroscopy.

Neutrons and light do not probe the same sample properties. With the exception of magnetic scattering, neutrons probe nuclear motions. In contrast, infrared and Raman spectroscopy relates to motion-induced reorientations and deformations of electron shells, i.e., to changes in molecular dipole moment and polarizability. Models, therefore, have to be developed for the coupling between the physical quantities studied by neutrons and light.

\section{Summary of the Performance of High-Resolution Instruments}

From the technical information provided about UCN instruments (Sec. V) and other instruments such as neutron reflectometers (Sec. VII.A), NSE spectrometers (Sec. VII.B), and optical devices (Sec. VII.C), one may attempt to compare the performance of these instruments.

For purely elastic reflectivity studies, grazing-angle neutron reflectometers have a higher sensitivity over a wider Q range than UCN instruments. On the other hand, UCN instruments provide reflectivity measurements with a resolution better than $1 \mathrm{neV}$, which is today beyond the reach of grazing-angle reflectometers. The advent of future UCN sources that could be more intense by two orders of magnitude than existing ones would improve the sensitivity of the UCN gravity diffractometer to reflectivity measurements down to about $10^{-6}$, which is the present sensitivity of SPEAR. But the very small $Q$ range of the UCN gravity diffractometer $\left(0.01 \AA^{-1}<\mathrm{Q}<0.015 \AA^{-1}\right)$ would permit the use of this increased sensitivity over a limited range of reflectivities and only for a limited number of samples: for example, those having a very small positive or a negative UCN potential. Also, the use of the TOF method gives an overwhelming advantage to the TOF grazingangle reflectometers compared with the UCN gravity diffractometer, which is used in point-by-point measurements. But TOF grazing-angle neutron reflectometers are not suited for quasi-elastic scattering studies. 
The instruments that can be used in quasi-elastic scattering studies can be compared in the Q- $\omega$ plane, as in Fig. 32. The lowest values of momentum transfers are now obtained with optical spectroscopy $\left(\mathrm{Q}<5 \times 10^{-3} \AA^{-1}\right)$. The existing NSE spectrometers can achieve resolutions down to about $8 \mathrm{neV}$ at $8 \AA$ over $Q$ values ranging from $0.015 \AA^{-1}$ to $1.35 \AA^{-1}$, but they can achieve resolutions of only $330 \mathrm{neV}$ at $4 \AA$ over $\mathrm{Q}$ values ranging from $0.03 \AA^{-1}$ to $2.7 \AA^{-1}$ (these figures are for the NSE instrument IN11.) At the present time there is a gap between $0.015 \AA^{-1}$ and $5 \times 10^{-3} \AA^{-1}$ for the Q ranges covered by NSE and optical spectrometers. This gap is partially filled by UCN instruments, which have excellent resolution (especially the gravity diffractometer) but over a very limited $Q$ range. This gap may also be filled in the next few years by the new generation of NSE spectrometers (IN15) whose $\mathrm{Q}$ range ( $\mathrm{Q}>10^{-3} \AA^{-1}$ ) may overlap with that of optical techniques. However, the resolution of the UCN gravity diffractometer will still remain far better than that of any other neutron instrument. As far as the count rate is concerned, the UCN diffractometer used with existing UCN sources has a count rate about one order of magnitude below that of IN11 in spite of the fact that this diffractometer can be used with much larger samples without deterioration of the resolution. With more intense UCN sources, a UCN diffractometer could be operated with a count rate comparable with that of IN11 and perhaps of IN15.

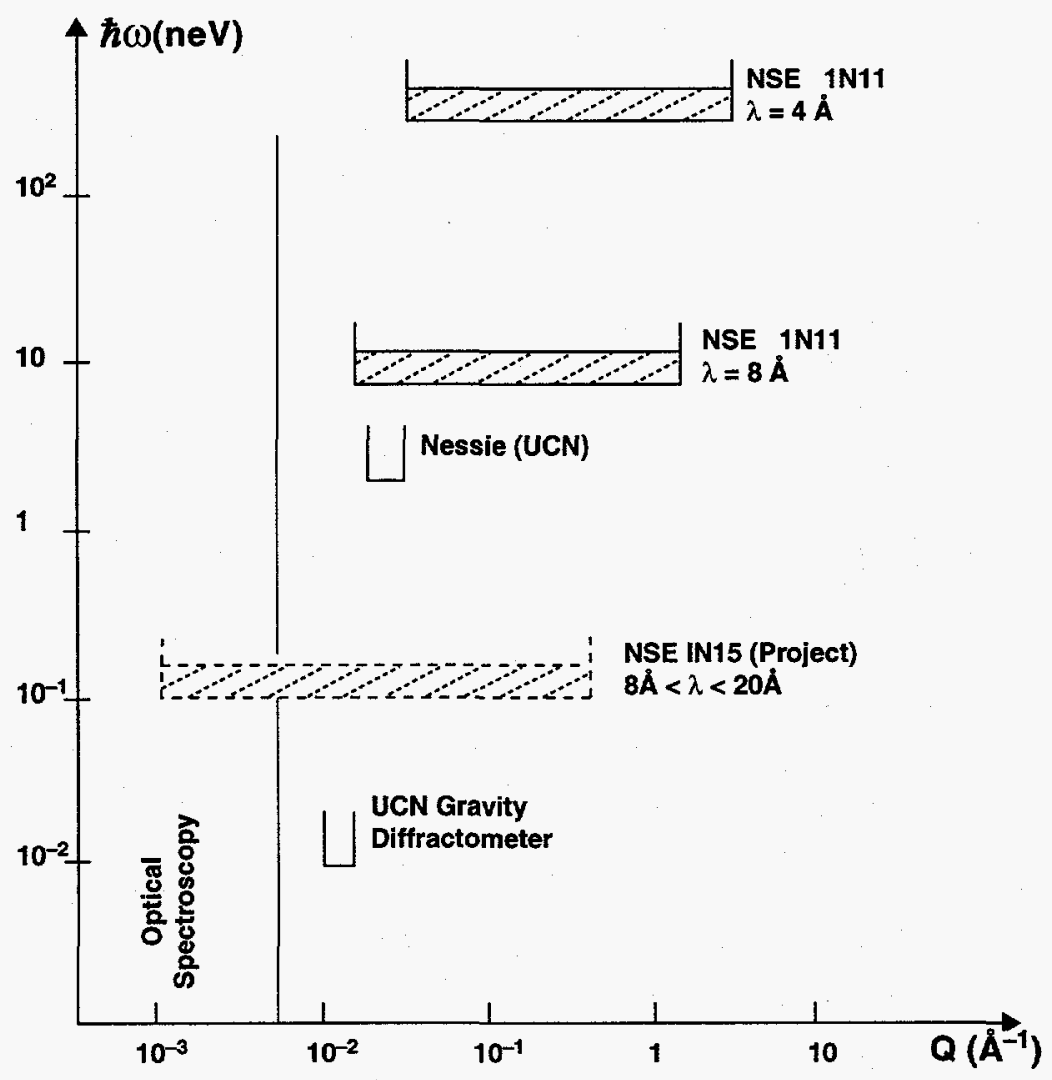

Fig. 32. Comparison of some high-resolution instruments in the $Q-\hbar \omega$ plane. The range $Q<5 \times 10^{-3} \AA^{-1}$ is covered by optical spectroscopy. The best possible spectral resolution achieved with INII is shown as a function of $Q$ for $\lambda=4 \AA$ and $\lambda=8 \AA$. Shaded areas correspond to samples of different sizes. The performance of the instrument project IN15 is shown as a dashed line for $8 \AA<\lambda<20 \AA$. The performance of NESSIE and of the neutron gravity diffractometer is also shown for comparison. The resolutions of these two UCN instruments are directly comparable with the spectral resolutions of the NSE instruments. 


\section{NEUTRON MICROSCOPES}

UCNs are also used in neutron microscopes. The idea of a neutron microscope was first put forward in the early 1970s (Frank 1972). Several arrangements of mirror-optics systems have been designed and tested for performing a three-dimensional focusing of very cold neutrons and UCNs (Frank 1992). Most of the tests were made with slits and sharp edges, but in a few cases some macroscopic neutron images of specimens have also been obtained. This discussion is restricted here to UCN microscopes.

In contrast to all other probes used in microscopy, the neutrons used in a UCN microscope interact with the nuclei in the specimen under study, except for magnetic samples. The neutron contrast obtained in a UCN microscope, therefore, reflects the nuclear composition of the sample and is different from that obtained with other radiations. Because UCN trajectories are strongly affected by the earth's gravitational field, a UCN microscope will be subject to specific gravitational aberrations, which have to be corrected in an achromatic instrument. The simplest type of UCN microscope is composed of two mirrors. The most recent results with UCN microscopes were obtained at the two most intense UCN sources in the world installed at the ILL (France) and Gatchina (Russia).

A schematic of the UCN microscope used at the ILL appears in Fig. 33 (Steyerl and Malik 1986, Steyerl et al. 1988, Herrmann et al. 1985). The microscope is composed of a primary large, concave, parabolic mirror (radius of curvature $R_{1}=585 \mathrm{~mm}$, diameter $\phi=200 \mathrm{~mm}$, coated with ${ }^{28} \mathrm{Ni}$ ) and a second smaller, convex, spherical mirror (radius of curvature $R_{2}$ ranging from $0.58 \mathrm{~m}$ to $1.6 \mathrm{~m}$, diameter $\phi=45 \mathrm{~mm}$, coated with natural $\mathrm{Ni}$ ). These mirrors are situated atop each other. They have the same vertical axis, and their focal points are at the same location (confocal arrangement). After reflection by these two mirrors, the UCNs are focused onto a small image mirror $\left(17 \times 17 \mathrm{~mm}^{2}\right)$, which reflects the UCNs to a $\mathrm{BF}_{3}$ detector through an inclined guide tube. Scanning the image is achieved by moving the image mirror in the image plane. The design, including a suitable choice of dimensions and the use of the highest point of the parabolic trajectory, makes possible the compensation of the two lowest-order chromatic aberrations. Higher-order chromatic aberrations cannot be eliminated, but they can be reduced by a coarse monochromatization of the UCN beam at the entrance of the microscope. Geometrical aberrations were reduced by the use of a parabolic shape for the primary mirror, which is responsible for most of the magnification.

Magnification factors of up to 240 with a spatial resolution of about $10 \mu \mathrm{m}$ were obtained with this instrument installed at the ILL. The magnification factor can be adjusted by varying the radius $R_{2}$ of the second mirror. An illustration of these results is given in Fig. 34 for a $0.4 \times 2 \mathrm{~mm}^{2}$ slit scanned by the image mirror. There were no images of more realistic objects made with this instrument.

Another two-mirror UCN microscope was developed in Russia and was used at the UCN beam of the Gatchina Institute of Nuclear Physics. This beam has a flux that is comparable with that of the ILL (Arzumanov et al. 1986). The Gatchina system is composed of two concave mirrors with radii of curvature $R_{1}$ and $R_{2}$ of about 20 and $30 \mathrm{~cm}$, respectively (Fig. 35). A thin layer of a ${ }^{48} \mathrm{Ni}-\mathrm{Mo}$ alloy is vacuum-deposited on these two mirrors, which makes total reflection possible for all neutrons with velocities below $8 \mathrm{~m} / \mathrm{s}$. The sample under study is located at the focal point of the first mirror. The distance $\mathrm{d}$ between the two mirrors satisfies the following condition:

$$
R_{1}+R_{2}=6 d
$$




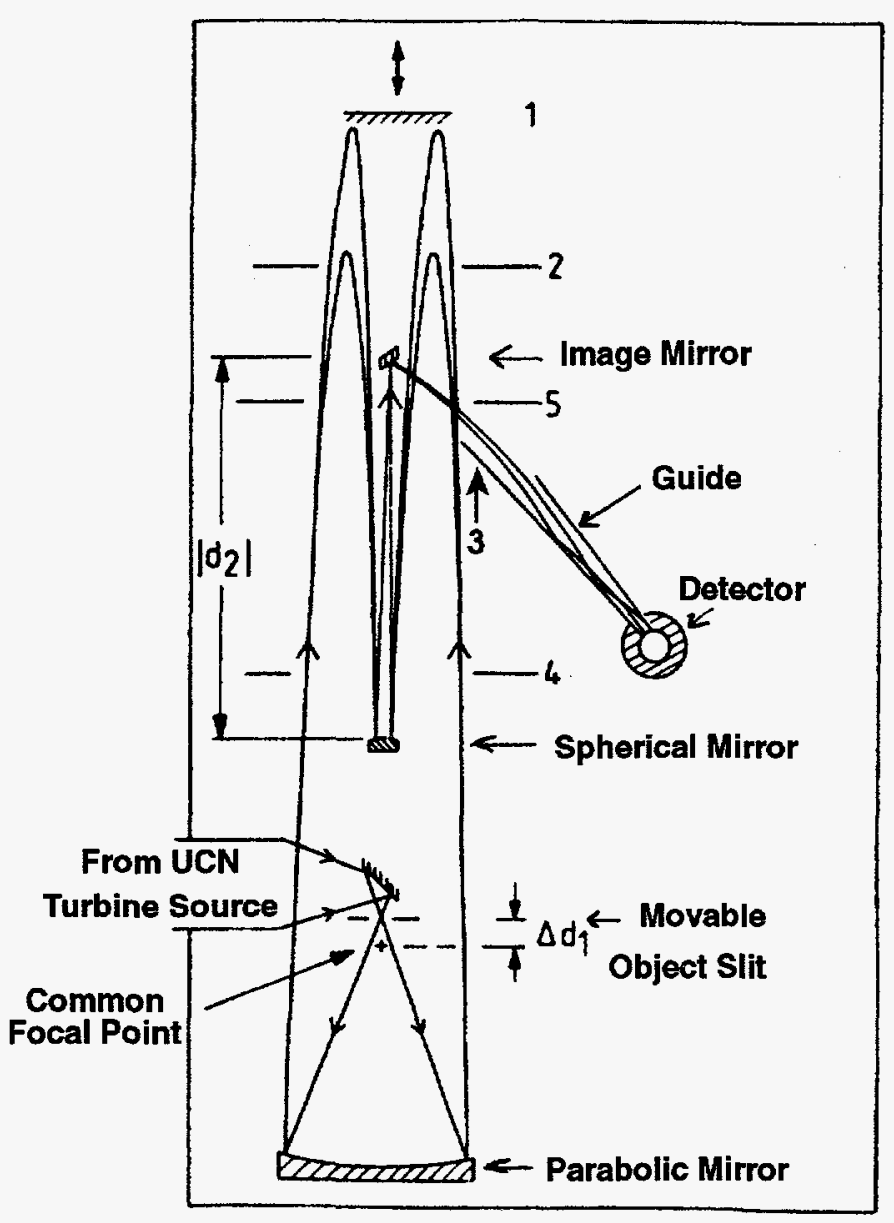

Fig. 33. Schematic of the two-mirror UCN microscope installed at the ILL. Beam tailoring and reflection of erratic neutrons are achieved with beam stops, numbered 1 to 5 (Steyerl et al. 1988). In this figure, the object is a movable slit.

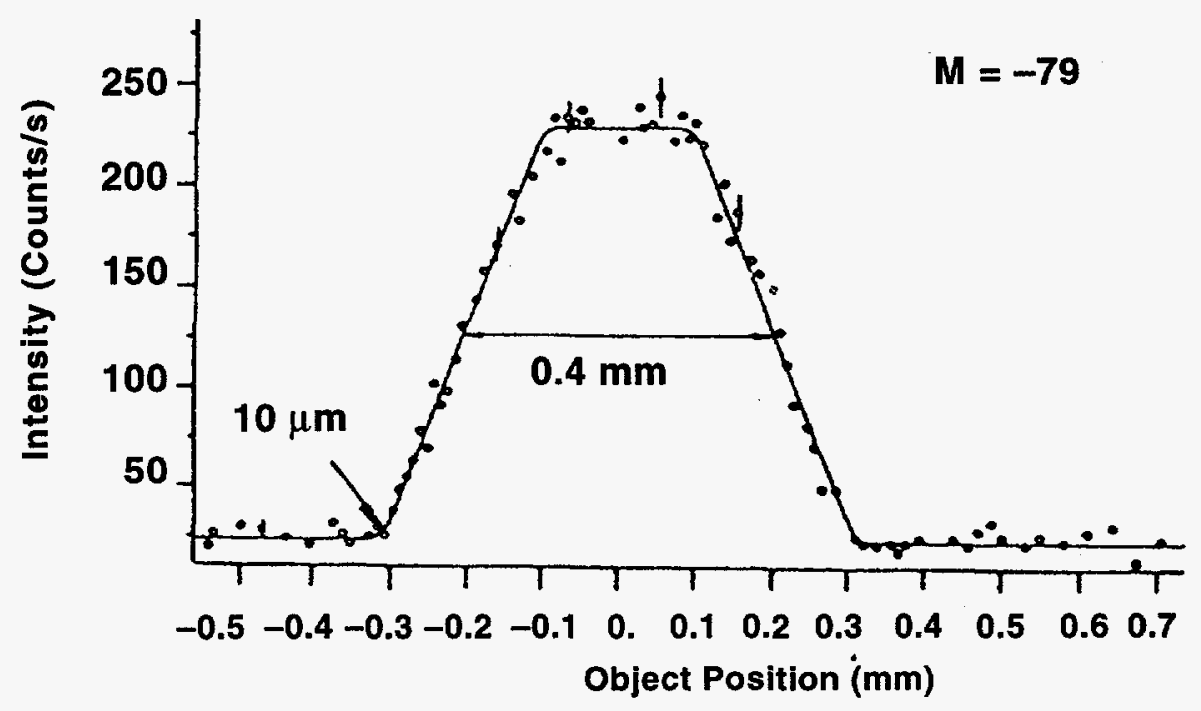

Fig. 34. Example of the results obtained with the UCN microscope shown in Fig. 33. A slit of $0.4 \mathrm{~mm}$ in width and $2 \mathrm{~mm}$ in length is scanned by the microscope. The setting of the instrument in this measurement was the following: $R_{2}=1.6079 \mathrm{~m}, \Delta d_{1}=0.29 \mathrm{~mm}, d_{2}=924.2 \mathrm{~mm}, M=-79$, and a UCN characteristic velocity of $(6.68 \pm 0.5) \mathrm{m} / \mathrm{s}$. The edge of the observed trapezoid is determined by the effective image mirror size $q=16.2 \mathrm{~mm}$ and the magnification factor $M=-79$. The aberration limit of $10 \mu \mathrm{m}$ to the resolution is derived from the corner blurring (Steyerl et al. 1988). 


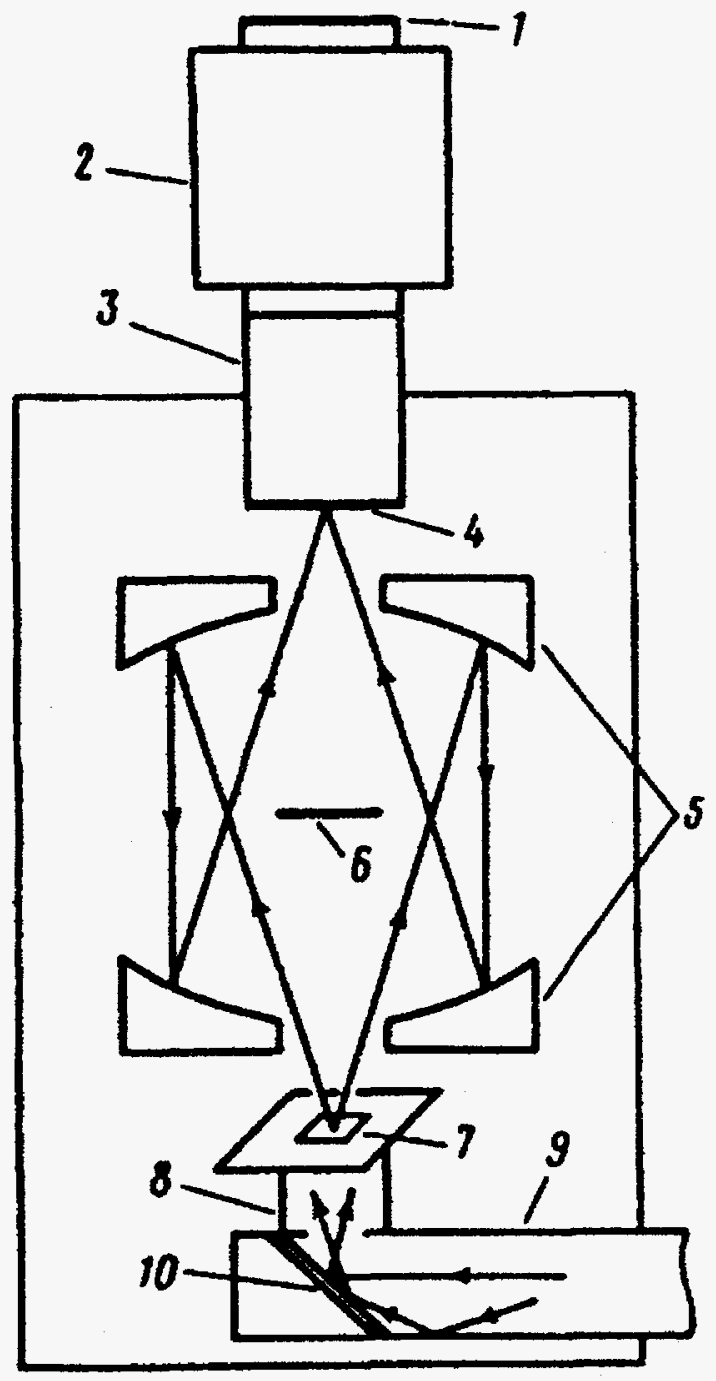

Fig. 35. Schematic of the two-mirror UCN microscope installed at the UCN source of the Gatchina Institute of Nuclear Physics (Russia):

(1) photographic film, (2) image converter,

(3) optical fiber, (4) scintillator sensitive to UCNs,

(5) concave mirrors of the optical system,

(6) shield against direct radiation, (7) sample, $(8,9)$ neutron ducts, and (10) auxiliary system (Arzumanov et al. 1986).

which provides first-order achromatization in both magnification and position. A UCN detector is placed in the image plane where the optical magnification $M$ is about $M=1.4$. The UCNs are detected in a thin coating of $\mathrm{LiF}$ through the ${ }^{6} \mathrm{Li}(\mathrm{n}, \alpha)$ reaction. The UCNs can easily penetrate the $\mathrm{LiF}$ coating because it contains about equal amounts of the two ${ }^{6} \mathrm{Li}$ and ${ }^{7} \mathrm{Li}$ isotopes, which have scattering lengths of comparable amplitude but of opposite signs. The $\alpha$-particles from the ${ }^{6} \mathrm{Li}(\mathrm{n}, \alpha)$ reaction are detected in a thin layer of a $\mathrm{ZnS}$ scintillator placed against the LiF coating. The light from the scintillations is extracted with an optical-fiber bundle and intensified by an image converter before being recorded on film.

An illustration of the results obtained with this microscope is given in Fig. 36. These results were obtained from a sample made of nickel (which has a high reflection for UCNs) formed by photolithography on a silicon wafer (which has a low reflection for UCNs). The spatial resolution was about $200 \mu \mathrm{m}$ after an exposure time of $2.5 \mathrm{~h}$. This first UCN image of a two-dimensional structure demonstrates the existence of a nuclear contrast in UCN optics (Arzumanov et al. 1986). 

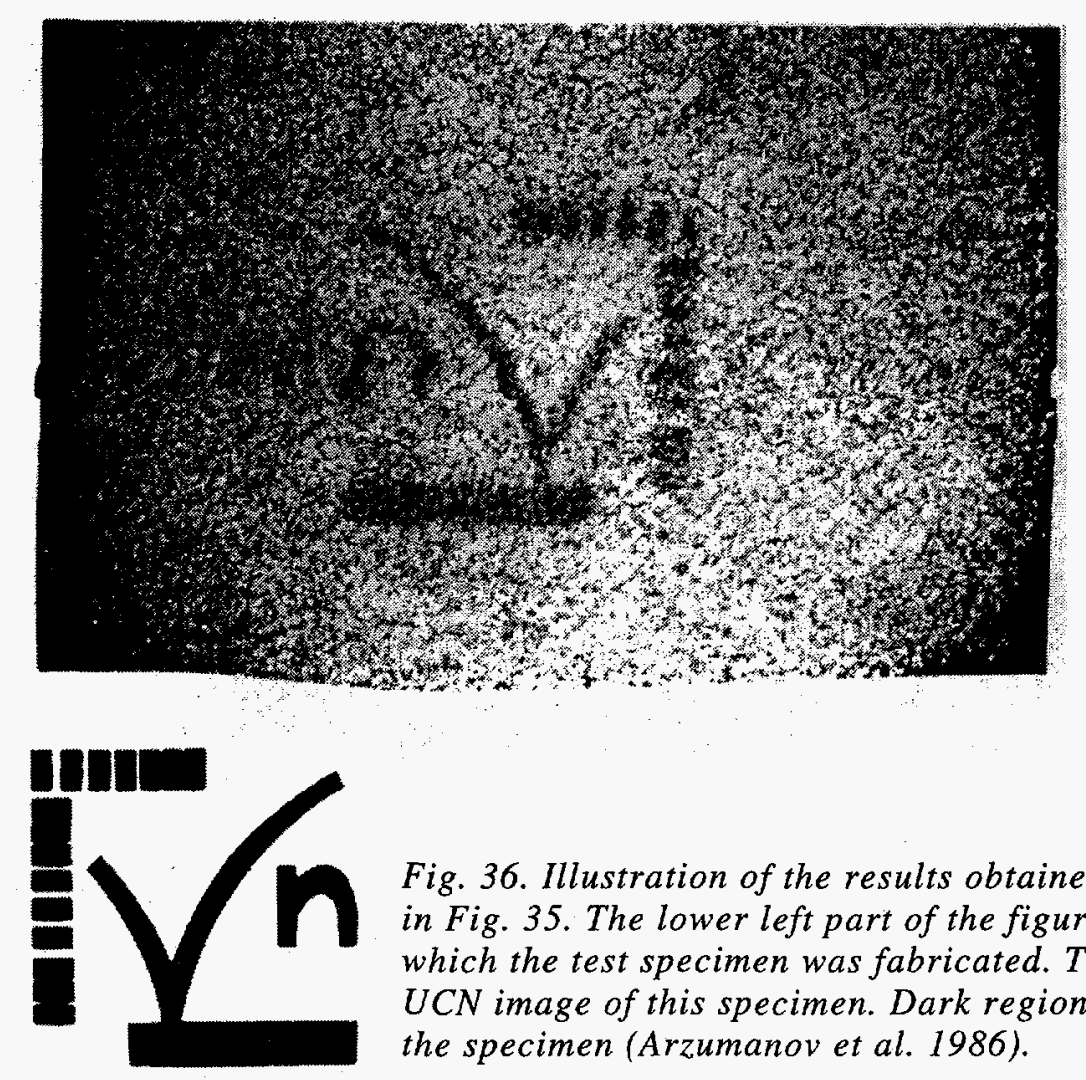

Fig. 36. Illustration of the results obtained with the UCN microscope shown in Fig. 35. The lower left part of the figure shows the phototemplate from which the test specimen was fabricated. The top part of the figure shows the UCN image of this specimen. Dark regions correspond to nickel coating in the specimen (Arzumanov et al. 1986).

Improvements of UCN microscopy include the use of more complicated mirror systems such as four-mirror ones (Arzumanov et al. 1984, Steyerl et al. 1988), which have better optical performance than two-mirror systems. For example, four-mirror instruments can be designed to be aplanatic, to eliminate longitudinal aberration to third order, and to eliminate transverse aberration to second order. The aberration limit of such systems could be of the order of a few $\mu \mathrm{m}$.

Another method proposed to reduce gravity aberrations is to compensate for gravity by applying a magnetic field in the region of the UCN trajectories (Skachkova and Frank 1981). More precisely, gravity can be canceled by using a suitable magnetic field gradient that would obey the following relation:

$$
\vec{\nabla}\left(\mu_{\mathbf{n}} \cdot \mathbf{B}\right)+\mathrm{mgk}=0
$$

This method requires the use of polarized neutrons and a uniform magnetic field gradient of $1.7 \times 10^{-2} \mathrm{Tcm}^{-1}$. Although, for practical reasons, such a large magnetic field gradient cannot be applied to the entire system, spatial resolutions of a few $\mu \mathrm{m}$ look possible (Golub et al. 1991).

There were plans to use these UCN microscopes to study surface magnetism and spatial distribution of hydrogenous structures in biological specimens, but apparently these projects never materialized. The possibility of exploiting the potential of these instruments depends crucially on the development of more intense UCN sources. For a resolution of $1 \mu \mathrm{m}$, the ILL UCN source would give a flux of $6 \times 10^{-5} \mathrm{n} / \mu \mathrm{m}^{2} \mathrm{~s}$, i.e., a count rate of $0.2 \mathrm{UCN}$ per hour for a $\mu \mathrm{m}^{2}$ resolution element. A significant increase in the strength of $\mathrm{UCN}$ sources is, therefore, necessary to make full use of the potential of UCN microscopes. 


\section{CONCLUSIONS}

This survey shows the interest that more intense UCN sources would present for condensed-matter studies. Of the two UCN instruments considered here, the UCN gravity diffractometer has performance significantly superior to that of the UCN spectrometer NESSIE. This diffractometer has much better resolution, a higher count rate, and is less bulky and much easier to operate than NESSIE.

A UCN gravity diffractometer occupies a special niche in the set of neutron instruments used for condensed-matter studies because of its excellent resolution in a narrow but well located range of $Q$ values. This excellent resolution, which has been demonstrated in many textbook experiments in quantum mechanics, surpasses that of any other existing neutron instrument and of new instruments foreseeable in the near future. However, purely elastic reflectivity studies usually necessitate measurements over a wide range of reflectivities (down to very low values of about $10^{-6}$ ) and momentum transfers rather than measurements with neV resolution in a narrow range of $Q$ values. Such wide ranges of reflectivity and momentum transfers are presently out of the reach of UCN instruments. An increase in UCN-beam intensity by two orders of magnitude, which is envisaged for the next generation of UCN sources, would improve the sensitivity of reflectivity measurements with UCN instruments by the same amount. This sensitivity would then match that of existing grazing-angle neutron reflectometers. But reflectivity measurements with UCN instruments would still be limited in range by the small and narrow window of $Q$ values available with these instruments and would also be restricted to a small number of samples that are restricted by their UCN potential. Moreover, the use of the TOF method gives a tremendous advantage to TOF grazing-angle reflectometers.

In spite of these shortcomings, the excellent resolution of UCN instruments could be used in some specific cases: for example, in the study of thick films with a critical edge below $\mathrm{Q}=0.01 \AA^{-1}$. Film thicknesses of $\approx 5,000 \AA$ are the upper limit of what can be examined with typical reflectometers in use today. But the narrow and closely spaced fringes obtained in reflectometry of thick films could be explored and resolved with the excellent resolution of UCN instruments, which is approximately one order of magnitude better than the resolution of standard reflectometry. Density profiles below surfaces could then be determined up to length scales of several $\mu \mathrm{m}$. This type of study could be applied to several types of industrial problems, which are not accessible with conventional reflectometers. Such applications might include metallic passivation layers (used to prevent oxidation or corrosion of surfaces), paints, and more general corrosion problems, to name a few.

The study of magnetic surfaces should a priori be an asset for UCN instruments because of the ease with which incident UCNs can be polarized and the polarization of scattered UCNs analyzed. But this apparently interesting possibility still remains largely unexplored, probably because of the developments of competitive, intense sources of polarized electrons, which can be used for similar purposes (Dubbers 1995). A more detailed study of the relative merits of polarized electrons and UCNs for the study of magnetic surfaces still remains to be done.

A UCN gravity diffractometer could also be used in the field of quasi-elastic neutron scattering, which cannot be carried out with TOF grazing-angle reflectometers. Quasi-elastic scattering can presently be studied with optical spectrometers below $\mathrm{Q}=5 \times 10^{-3} \AA^{-1}$ and with NSE spectrometers above $\mathrm{Q}=0.015 \AA^{-1}$. UCN instruments 
partially fill the Q-value gap between optical and NSE instruments. Moreover, the resolution of a UCN gravity diffractometer is two to three orders of magnitude better than that of existing NSE spectrometers. Therefore, a UCN instrument can very well supplement optical and NSE spectrometers, but it cannot replace them because of its too limited Q range. UCN instruments can also supplement these two types of instruments for other reasons. First, neutrons and EM waves do not probe the same microscopic sample properties, and models are needed to relate neutron and optical experimental results. Second, although UCN and NSE spectrometers use the same probe, they do not measure the same physical quantity. UCN instruments directly measure the scattering function $S(\vec{Q}, \omega)$, whereas NSE instruments give access to the intermediate scattering function $F(\vec{Q}, t)$, which is the Fourier transform of $S(\vec{Q}, \omega)$. Finally, from a more pragmatic point of view, NSE instruments accept samples only in the vertical position, whereas a UCN gravity diffractometer can accommodate both horizontal and vertical samples. UCN instruments can therefore be used in the study of liquid and other samples that can be analyzed only in the horizontal position. Therefore, UCN and NSE instruments can supplement each other, depending on the nature of the physical processes and on the characteristics of the samples under study.

In the future, the new generation of NSE spectrometers (IN15) under construction is expected to bridge the gap in $Q$ values between those of the existing NSE and optical spectrometers. But the resolution of these new NSE instruments will still be broader by one order of magnitude than that of the UCN gravity diffractometer, and the general complementary aspects between optical, NSE, and UCN instruments already discussed should still remain valid.

The main weakness of UCN instruments resides in their low count rate, which is a consequence of the low intensity of existing UCN sources. If more intense UCN beams were built, they would generate a renewed interest in UCN instruments of the gravitydiffractometer type, whose count rate would then become comparable with that of NSE spectrometers. Quasi-elastic scattering measurements of increased sensitivity would give access to slow motions of macromolecules in polymers and biological samples. Although these samples would need large areas to compensate for the low intensity of UCN instruments, they could be made very thin because of the small penetration depth of UCNs, with the consequence that the overall volume of each of these samples would remain small.

Among the new UCN sources under consideration, those of superfluid helium could produce UCN densities inside the liquid-helium bath several orders of magnitude higher than those of existing UCN sources (Golub and Lamoreaux 1994, Lamoreaux 1995a, Golub 1995). Although the main use of this type of UCN source would be for fundamental physics (for example, measurement of the neutron electric dipole moment), a small hole in the wall of such a source would produce a UCN leak source with a high brilliance, which could be used with a UCN spectrometer of the NESSIE type (Lamoreaux 1995b).

Finally, more intense UCN sources could also reactivate the field of neutron microscopy for the study of macroscopic objects and supplement microscopy results obtained with other probes such as electrons or EM radiation. The use of polarized UCNs in neutron microscopy would also make it possible to explore the microscopy of magnetic structures. 


\section{ACKNOWLEDGMENTS}

The author is very much indebted to Hans Fauenfelder, Rex Hjelm, and Gregory Smith for their contributions on biopolymers, polymers, and grazing-angle reflectometry, respectively. The author also very much appreciated the useful information obtained from Roland Gähler, Robert Golub, Erwin Gutsmiedl, Steve Lamoreaux, and Albert Steyerl. Last but not least, the author is very grateful to Robert Haight and Gregory Smith for the comments and corrections they proposed after a careful reading of the manuscript, and to Ileana Buican and Vaikunth Stewart for their thorough editorial help.

\section{REFERENCES}

Arzumanov, S. S., S. V. Masalovich, et al., "Image Detection in a Neutron Microscope," JETP Lett. 44, 271 (1986).

Arzumanov, S. S., S. V. Masalovich, et al., "Many-Mirror Optical System for Obtaining a Neutron Image - a Possible Prototype of a Neutron Microscope," JETP Lett. 39, 590 (1984).

Bée, M., Quasielastic Neutron Scattering (Adam Hilger, Bristol \& Philadelphia, 1988).

Dagleish, P. A., J. B. Hayter, and F. Mezei, “The IN11 Neutron-Spin-Echo Spectrometer," in Neutron Spin Echo, Proceedings of a Laue-Langevin Institute Workshop, Grenoble, October 15-16, 1979, F. Mezei, Ed. (Springer-Verlag, Berlin, Germany, 1980).

Dubbers, D., Heidelberg Universität, private communication, August 1995.

Felcher, G. P., "Magnetic Depth Profiling Studies by Polarized Neutron Reflection," Physica B 192, 137 (1993).

Felcher, G. P., R. O. Hilleke, R. K. Crawford, et al., "Polarized Neutron Reflectometer: A New Instrument to Measure Magnetic Depth Profiles," Rev. Sci. Instrum. 58, 609 (1987).

Frank, A. I., "Microscopy with Ultracold Neutrons" in Neutron Optical Devices and Applications, C. F. Marjkzak and J. Wood, Eds. (SPIE, Bellingham, Washington, 1992), Vol. 1738, p. 323.

Frank, A. I., "Some New Aspects of Neutron Optics," Priroda 9, 24 (1972). (This article is written in Russian.)

Frauenfelder, H., Los Alamos National Laboratory, private communication, January 1996.

From the Tip of the LANSCE, a Newsletter from Manuel Lujan Jr. Neutron Scattering Center, No. 15, Spring 1993, Los Alamos National Laboratory document.

Gähler, R., Technische Universität, München, private communication, December 1995. 
Goldstone, J. A., "The Manuel Lujan Jr. Neutron Scattering Center," in Neutron Scattering in Materials Science II, D. A. Newmann, T. P. Russell, and B. J. Wuensch Eds. (Material Research Society, Pittsburgh, Pennsylvania, 1995), Vol. 376, pp. 13-24.

Golub, R., "UCN Scattering” (Ultracold Neutron Workshop, Santa Fe, NM, December 6-8, 1995).

Golub, R., "Ultra-Cold Neutrons: Their Role in Studies of Condensed Matter," Rev. Mod. Phys. 68, 329 (1996).

Golub, R., and S. K. Lamoreaux, "Neutron Electric-Dipole Moment, Ultracold Neutrons, and Polarized "He," Phys. Rep. 237, 1 (1994).

Golub, R., and J. M. Pendlebury, "Ultra-Cold Neutrons," Rep. Progr. Phys. 42, 440 (1979).

Golub, R., D. J. Richardson, and S. K. Lamoreaux, Ultra-Cold Neutrons (Adam Hilger, Bristol, Philadelphia, and New York, 1991).

Golub, R., W. Mampe, et al., "Ultracold Neutrons," Sc. Am. 240 (6), 134 (1979).

Herdin, R., A. Steyerl, A. R. Taylor, et al., "Experiment on the Efficient Polarization of Ultracold Neutrons," Nucl. Instrum. Methods 148, 353 (1978).

Herrmann, F., "Reflektometrie mit Ultrakalten Neutronen," Diplomarbeit, Grenoble (1990). (This work is unpublished.)

Herrmann, P., K.-A. Steinhauser, R. Gähler, et al., "Neutron Microscope," Phys. Rev. Lett. 54, 1969 (1985).

Hjelm, R., Los Alamos National Laboratory, private communication, January 1996.

Ibel, K., Ed., Guide to Neutron Research Facilities at the ILL (Institut Laue-Langevin, Grenoble, France, 1994).

Ignatovich, V. K., The Physics of Ultra-Cold Neutrons (Clarendon Press, Oxford, 1990).

Koester, L., W. Washkowski, and A. Klüver, "Neutron Scattering Lengths and Neutron-Electron Interaction," Physica B 137, 282 (1986).

Lamoreaux, S. K., "A New Proposed Technique to Search for the Neutron Electric Dipole Moment (EDM) by Using Polarized ${ }^{3} \mathrm{He}$ and Ultracold Neutrons Stored Together in Superfluid ${ }^{4} \mathrm{He}$ " (Ultracold Neutron Workshop, Santa Fe, NM, December 6-8, 1995a).

Lamoreaux, S. K., University of Washington, private communication, October $1995 \mathrm{~b}$. 
Lekner, J., Theory of Reflection (Martinus Nijhoff Publishers, Dordrecht, the Netherlands, 1987).

Mezei, F., Ed., Neutron Spin Echo (Springer-Verlag, Berlin, 1980).

Middendorf, H. D., "Inelastic Scattering from Biomolecules: Principles and Prospects," in Neutrons in Biology, Benno P. Schoenborn, Ed. (Plenum Press, New York, 1984).

Pendlebury, J. M., "Fundamental Physics with Ultracold Neutrons," Annu. Rev. Nucl. Part. Sci. 43, 687 (1993).

Pfeiffer, W., G. Schlossbauer, W. Knoll, et al., "Ultracold Neutron Scattering Study of Local Lipid Mobility in Bilayer Membranes," J. Phys. France 49, 1077 (1988).

Russell, T. P., "X-Ray and Neutron Reflectivity for the Investigation of Polymers," Materials Science Reports 5, 171 (1990).

Scheckenhofer, H., and A. Steyerl, "A Gravity Diffractometer for Ultracold-Neutron Optics," Nucl. Instrum. Methods 179, 393 (1981).

Scheckenhofer, H., and A. Steyerl, "Diffraction and Mirror Reflection of Ultracold Neutrons," Phys. Rev. Lett. 39, 1310 (1977).

Skachkova, O. S., and A. I. Frank, "Gravitational Distortions in Neutron-Optical Systems and Their Reduction by Means of Nonuniform Magnetic Fields," JETP Lett. 33, 203 (1981).

Smith, G., Los Alamos National Laboratory, private communication, January 1996.

Steinhauser, K. A., A. Steyerl, et al., "Observation of Quasibound States of the Neutron in Matter,” Phys. Rev. Lett. 44, 1306 (1980).

Steyerl, A., “A High-Resolution Neutron Gravity Spectrometer,” Z. Phys. B 30, 231 (1978).

Steyerl, A., "A 'Neutron Turbine' as an Efficient Source of Ultracold Neutrons," Nucl. Instrum. Methods 125, 461 (1975).

Steyerl, A., "A Time-of-Flight Spectrometer for Ultracold Neutrons," Nucl. Instrum. Methods 101, 295 (1972b).

Steyerl, A., "Effect of Surface Roughness on the Total Reflection and Transmission of Slow Neutrons," Z. Phys. 254, 169 (1972a).

Steyerl, A., "Measurements of Total Cross Sections for Very Slow Neutrons with Velocities from $100 \mathrm{~m} / \mathrm{s}$ to $5 \mathrm{~m} / \mathrm{s}$," Phys. Lett. B 29, 33 (1969).

Steyerl, A., "Production and Properties of Ultracold Neutrons," in The Neutron and Its Applications, P. Schoefield, Ed. (The Institute of Physics, Bristol and London, 1983), p. 177. 
Steyerl, A., "Ultracold Neutrons: Production and Experiments," Physica B 156/157, 528 (1989).

Steyerl, A., and S. S. Malik, "New Developments in Cold- and Ultracold-Neutron Research," Physica B 137, 270 (1986).

Steyerl, A., and S. S. Malik, "Sources of Ultracold Neutrons," Nucl. Instrum. Methods A 284, 200 (1989).

Steyerl, A., T. Ebisawa, et al., "Experimental Study of Macroscopic Coupled Resonators for Neutron Waves," Z. Phys. B 41, 283 (1981).

Steyerl, A., W. Drexel, T. Ebisawa, et al., "Neutron Microscopy," Revue Phys. Appl. 23, 171 (1988).

Steyerl, A., B. Gmal, K. A. Steinhauser, et al., "Features and Performances of a Gravity Spectrometer for Ultracold Neutrons," Z. Phys. B 50, 281 (1983).

Steyerl, A., S. S. Malik, A. C. Nunes, et al., "Reflectivity and Profile Studies in Ultracold Neutron Mirror Reflection and Grating Diffraction," in Neutron Optical Devices and Applications, C. F. Marjkzak and J. Wood, Eds. (SPIE, Bellingham, Washington, 1992), Vol. 1738 , p. 312.

Steyerl, A., H. Nagel, F. X. Schreiber, et al., "A New Source of Cold and Ultracold Neutrons," Phys. Lett. A 116, 347 (1986).

Timmins, P. A., and H. Lauter, Institut Laue-Langevin, France, private communication, January 1996. 


\section{LIST OF SYMBOLS}

b

$\mathrm{b}_{\text {coh }}$

$b_{\text {inc }}$

d

B

$\mathrm{B}_{\mathrm{T}}$

$\mathrm{B}_{\mathrm{z}}$

$\mathrm{B}_{\uparrow}$

$B_{\perp}$

$\mathrm{D}_{\mathrm{T}}$

E

$\mathrm{E}_{\mathrm{o}}$

$\mathrm{E}_{\mathrm{f}}$

$\mathrm{E}_{\mathrm{nz}}$

$\mathrm{F}_{\mathrm{coh}}(\overrightarrow{\mathrm{Q}}, \mathrm{t}) \quad$ intermediate coherent scattering function

$F_{\text {inc }}(\vec{Q}, t) \quad$ intermediate incoherent scattering function

$\mathrm{G}(\overrightarrow{\mathrm{r}}, \mathrm{t})$

$\mathrm{G}_{\mathrm{d}}(\overrightarrow{\mathrm{r}}, \mathrm{t})$

$\mathrm{G}_{\mathrm{s}}(\overrightarrow{\mathrm{r}}, \mathrm{t})$

k

$\mathbf{k}_{0}$

$\mathbf{k}_{\mathrm{f}}$

$\mathbf{k}_{\mathrm{c}}$

$\mathrm{k}_{\mathrm{zo}}$

$\mathbf{k}_{\mathrm{z} 1}$

$\mathrm{m}$

n

$\mathrm{P}_{\mathrm{n}}$

$\mathrm{P}_{\text {NSE }}$

$\mathrm{P}_{\mathrm{NSE}}^{0}$

$P_{\mathrm{NSE}}^{\exp }$

neutron scattering length

bound coherent scattering length

bound incoherent scattering length

magnetic induction in the sample

total magnetic induction

z-component of the magnetic induction

effective translational diffusion coefficient

neutron energy

incident-neutron energy

scattered-neutron energy number $\mathrm{n}$

space-time correlation function

distinct-correlation function

self-correlation function

neutron wave number

wave number of the incident neutron

wave number of the scattered neutron

critical wave number medium 1

neutron mass

index of refraction

neutron polarization
- thickness of the transition region described by a "soft potential"

- spatial periodicity of a periodic surface corrugation

- distance between two mirrors of a two-mirror UCN microscope

component of the magnetic induction parallel to the neutron spin

component of the magnetic induction perpendicular to the neutron spin

vertical energy of the incident neutron corresponding to quasi-bound state of

component along axis $\mathrm{z}$ of the wave number of the incident neutron

component along axis $\mathrm{z}$ of the wave number of the refracted neutron in

neutron polarization at the end of an NSE spectrometer

value of $\mathrm{P}_{\text {NSE }}$ for pure elastic scattering in the presence of experimental effects

value of $\mathrm{P}_{\mathrm{NSE}}$ obtained in a measurement of quasi-elastic scattering 


$\begin{array}{ll}\mathrm{Q} & \text { momentum transfer (in units of } \hbar) \\ \mathrm{r} & \text { reflectivity amplitude } \\ \mathrm{r}_{ \pm \pm} & \text {reflectivity amplitude of polarized neutrons by a magnetic sample } \\ \mathrm{R} & \text { reflectivity } \\ \mathrm{R}_{\mathrm{H}} & \text { equivalent hydrodynamic radius } \\ \mathrm{S}(\overrightarrow{\mathrm{Q}}) & \text { static structure factor } \\ \mathrm{S}_{\mathrm{coh}}(\overrightarrow{\mathrm{Q}}) & \text { coherent static structure factor } \\ \mathrm{S}_{\mathrm{inc}}(\overrightarrow{\mathrm{Q}}) & \text { incoherent static structure factor } \\ \mathrm{S}_{\mathrm{coh}}(\overrightarrow{\mathrm{Q}}, \omega) & \text { dynamic coherent structure factor } \\ \mathrm{S}_{\mathrm{inc}}(\overrightarrow{\mathrm{Q}}, \omega) & \text { dynamic incoherent structure factor } \\ \mathrm{t} & \text { transmission amplitude } \\ \mathrm{T} & \text { transmission } \\ \mathrm{U}_{\mathrm{ucn}} & \text { global UCN potential } \\ \mathrm{U}_{-} & \text {UCN potential for magnetic materials when the magnetic induction in the } \\ \mathrm{U}_{+} & \text {sample and the neutron magnetic moment are parallel } \\ \mathrm{v}^{\prime} & \text { UCN potential for magnetic materials when the magnetic induction in the } \\ \mathrm{v}_{\mathrm{c}} & \text { sample and the neutron magnetic moment are antiparallel } \\ \mathrm{V} & \text { neutron velocity in the sample } \\ \mathrm{z}_{\mathrm{i}} & \text { UCN critical velocity } \\ \Delta & \text { neutron potential } \\ \Delta \mathrm{E}_{\mathrm{inc}} & \text { thickness of layer i in a multilayer specimen } \\ \Delta \mathrm{E}_{\mathrm{ucn}} & \text { lateral correlation length } \\ \gamma_{\mathrm{n}} & \text { total width of the quasi-elastic scattering peak } \\ \eta & \text { resolution of a UCN reflectometer } \\ \lambda & \text { neutron gyromagnetic ratio } \\ \lambda_{\mathrm{c}} & \text { solvant viscosity } \\ \mu_{\mathrm{n}} & \text { neutron wavelength } \\ \theta \text { or } \theta_{1} & \text { neutron critical wavelength } \\ \theta_{1}^{\prime} & \text { neutron magnetic moment } \\ \theta_{2} & \text { neutron angle of incidence } \\ \theta_{\mathrm{c}} & \text { neutron angle of reflection } \\ \theta_{\mathrm{n}} & \text { neutron angle of refraction } \\ \rho & \text { critical angle of incidence } \\ \tau_{\mathrm{o}} & \text { neutron reflection angle of order } \mathrm{n} \\ \zeta_{\mathrm{z}} & \text { material density } \\ \varphi & \text { trapping time } \\ & \text { height variations (from a zero mean value) on a rough surface } \\ \text { neutron precession angle }\end{array}$


ANL

$\mathrm{CM}$

DNA

DMPC

DPPC

EISF

EM

IPNS

FRAP

FRM

FWHM

ILL

LANSCE

NESSIE

NSE

PNR

QELS

SPEAR

SPSS

TOF

UCN neutron total precession phase obtained with an NSE instrument total wavefunction of the neutron wavefunction of the incident neutron wavefunction of the reflected neutron for an nth-order reflection energy transfer during inelastic scattering (in units of $\hbar$ )

\section{LIST OF ACRONYMS}

atomic force microscopy

Argonne National Laboratory

center of mass

deoxyribonucleic acid

dimyristoyl phosphatidyl choline

dipalmitoyl phosphatidyl choline

elastic incoherent structure factor

electromagnetic

intense pulsed neutron source (at ANL)

photobleaching technique

Forschungs Reactor Munich

full width at half maximum

Institut Laue-Langevin

Los Alamos Neutron Science Center

Neutronen Schwerkraft Spektrometrie

neutron spin echo

polarized neutron reflectivity

quasi-elastic light scattering

surface profile analysis reflectometer

short-pulse spallation neutron source

time of flight

ultracold neutron 